\title{
Structures Illuminate Cardiac Ion Channel Functions in Health and in Long QT Syndrome
}

\author{
Kathryn R. Brewer ${ }^{1,2 \dagger}$, Georg Kuenze ${ }^{1,3 \dagger}$, Carlos G. Vanoye ${ }^{4}$, Alfred L. George Jr. , \\ Jens Meiler ${ }^{1,3,5,6}$ and Charles R. Sanders ${ }^{1,2 *}$ \\ ${ }^{1}$ Center for Structural Biology, Vanderbilt University School of Medicine Basic Sciences, Nashville, TN, United States, \\ ${ }^{2}$ Department of Biochemistry, Vanderbilt University, Nashville, TN, United States, ${ }^{3}$ Department of Chemistry, Vanderbilt \\ University, Nashville, TN, United States, ${ }^{4}$ Department of Pharmacology, Feinberg School of Medicine, Northwestern \\ University, Chicago, IL, United States, ${ }^{5}$ Department of Pharmacology, Vanderbilt University School of Medicine Basic \\ Sciences, Nashville, TN, United States, ${ }^{6}$ Institute for Drug Discovery, Leipzig University Medical School, Leipzig, Germany
}

OPEN ACCESS

Edited by:

Mounir Tarek,

Centre National de la Recherche Scientifique (CNRS), France

Reviewed by:

Sergei Noskov,

University of Calgary, Canada

Clemens Möller,

Hochschule Albstadt-Sigmaringen,

Germany

*Correspondence:

Charles R. Sanders

chuck.sanders@vanderbilt.edu

${ }^{\dagger}$ These authors have contributed equally to this work

Specialty section:

This article was submitted to

Pharmacology of lon Channels

and Channelopathies,

a section of the journal

Frontiers in Pharmacology

Received: 30 January 2020 Accepted: 09 April 2020

Published: 04 May 2020

Citation:

Brewer KR, Kuenze G, Vanoye CG, George AL Jr., Meiler J and Sanders CR (2020) Structures Illuminate Cardiac Ion Channel

Functions in Health and in

Long QT Syndrome.

Front. Pharmacol. 11:550.

doi: 10.3389/fphar.2020.00550
The cardiac action potential is critical to the production of a synchronized heartbeat. This electrical impulse is governed by the intricate activity of cardiac ion channels, among them the cardiac voltage-gated potassium $\left(K_{v}\right)$ channels $K C N Q 1$ and $h E R G$ as well as the voltage-gated sodium $\left(\mathrm{Na}_{\mathrm{v}}\right)$ channel encoded by SCN5A. Each channel performs a highly distinct function, despite sharing a common topology and structural components. These three channels are also the primary proteins mutated in congenital long QT syndrome (LQTS), a genetic condition that predisposes to cardiac arrhythmia and sudden cardiac death due to impaired repolarization of the action potential and has a particular proclivity for reentrant ventricular arrhythmias. Recent cryo-electron microscopy structures of human KCNQ1 and hERG, along with the rat homolog of SCN5A and other mammalian sodium channels, provide atomic-level insight into the structure and function of these proteins that advance our understanding of their distinct functions in the cardiac action potential, as well as the molecular basis of LQTS. In this review, the gating, regulation, LQTS mechanisms, and pharmacological properties of KCNQ1, hERG, and SCN5A are discussed in light of these recent structural findings.

Keywords: cardiac action potential, long QT syndrome, KCNQ1, hERG, SCN5A, structural biology

\section{INTRODUCTION}

The cardiac action potential is critical to proper heart function. Beginning with the activation of "pacemaker" cells, the action potential propagates through the atria and into the ventricles in a unidirectional waveform of excitation and relaxation, resulting in the coordinated expansion and contraction of heart tissue (Nerbonne and Kass, 2005). The action potential is governed by an intricate series of ion channel activities (Grant, 2009), including those of the KCNQ1 ( $\mathrm{K}_{V} \mathrm{LQT} 1$, $\left.\mathrm{K}_{\mathrm{V}} 7.1\right)$ and hERG $\left(\mathrm{KCNH} 2, \mathrm{~K}_{\mathrm{V}} 11.1\right)$ potassium channels and the SCN5A $\left(\mathrm{Na}_{\mathrm{V}} 1.5\right)$ sodium channel. Mutations in these three channels are the most frequent cause of congenital long QT syndrome (LQTS), a cardiac arrhythmia disorder that is one of the primary causes of sudden arrhythmic death syndrome (SADS) (Skinner et al., 2019). 
KCNQ1, hERG, and SCN5A each play a distinct role in generating the cardiac action potential (Figure 1), consequently producing distinct LQTS forms when mutated (Skinner et al., 2019). The initial upstroke is governed primarily by SCN5A, producing the $\mathrm{I}_{\mathrm{Na}}$ current that amplifies membrane depolarization and propagates the action potential (Moss and Kass, 2005; Skinner et al., 2019). Mutation in SCN5A causes LQTS type 3 (LQT3). hERG shapes both the plateau and the repolarization phases of the action potential, along with KCNQ1 in complex with the KCNE1 accessory protein (Moss and Kass, 2005; George, 2013). hERG and the KCNQ1-KCNE1 complex produce the rapid $\left(\mathrm{I}_{\mathrm{Kr}}\right)$ and slow $\left(\mathrm{I}_{\mathrm{Ks}}\right)$ delayed rectifier currents (Figures 1B, C), respectively. KCNQ1 mutation causes LQT1, and hERG, LQT2 (Skinner et al., 2019). Additional ion channels and transporters also shape the cardiac action potential (Skinner et al., 2019), among them $\mathrm{Ca}_{\mathrm{v}} 1.2$, NCX1, and Kir2.1. The roles of these channels in the cardiac action potential and cardiac arrhythmias have been reviewed elsewhere (Bidaud and Lory, 2011; Hancox et al., 2018; Meza et al., 2018; Vaidyanathan et al., 2018; Li et al., 2019). These channels are not typically causative for LQTS and will not be discussed here.

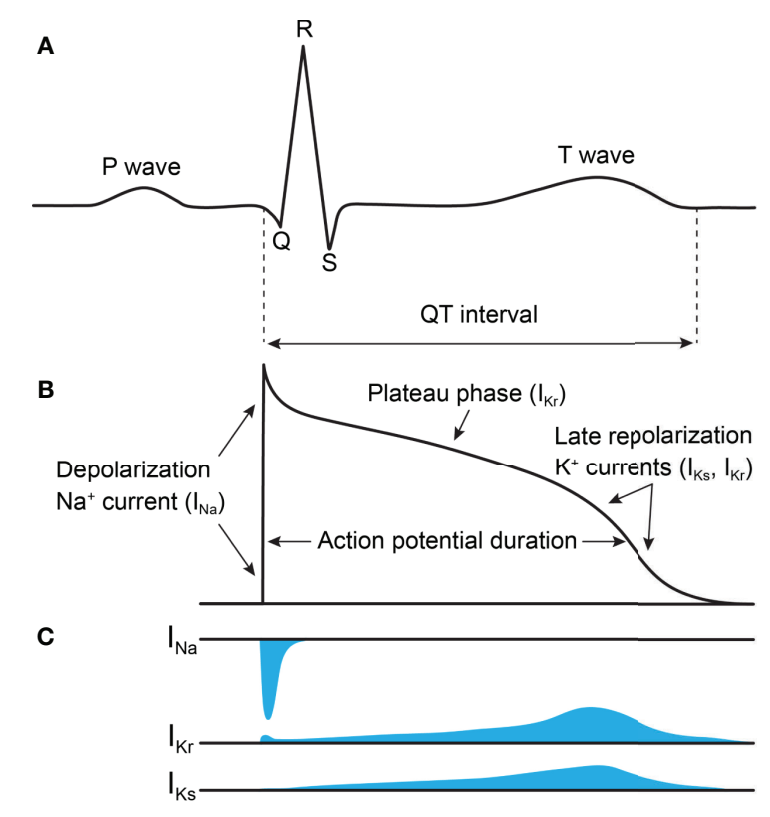

FIGURE 1 | Voltage-activated $\mathrm{Na}^{+}$and $\mathrm{K}^{+}$currents define the ventricular action potential and QT interval of the ECG. (A) ECG trace. The rapid upstroke of the ventricular action potential gives rise to the QRS complex. The duration of the QT interval is determined by the time of the ventricular repolarization. (B) Trace of the ventricular action potential. The rapidly activating and inactivating $I_{\mathrm{Na}}$ current drives membrane depolarization. Two $\mathrm{K}^{+}$currents, $I_{\mathrm{Ks}}$ and $\mathrm{I}_{\mathrm{Kr}}$, contribute most to the plateau phase and repolarization phase of the action potential, which reestablishes the membrane resting potential. (C) Time course of $I_{\mathrm{Na}}$, $I_{\mathrm{Kr}}$, and $\mathrm{l}_{\mathrm{Ks}}$ currents (not drawn to scale). Currents of other ion channels contributing to the action potential (e.g. $I_{\mathrm{Ca}, \mathrm{L}}, \mathrm{I}_{\mathrm{Kur}}$, and $\left.\mathrm{I}_{\mathrm{NCX}}\right)$ are not shown for clarity. This figure is inspired by figures in Moss and Kass (2005) and George (2013).
Structurally, the KCNQ1, hERG, and SCN5A channels belong to the voltage-gated ion channel superfamily and share a general transmembrane topology (Bezanilla, 2005; Dehghani-Samani et al., 2019). The transmembrane channel domain is composed of six helices per subunit in hERG and KCNQ1 (Figures 2A, B) or a monomeric tetrad repeat of linked 6-helix domains for SCN5A, each repeat exhibiting varying sequences, lengths, and tertiary folds (Figures 2C, D). The assembled channel is tetrameric (pseudo-tetrameric for SCN5A), with each channel domain composed of four voltage sensing domains (VSDs) surrounding a central pore domain (PD) (Figure 2D). The VSD is comprised of the first four transmembrane helices (S1S4) preceded by a small amphipathic helix — S0 — in both KCNQ1 and SCN5A (Jiang et al., 2020; Sun and Mackinnon, 2020). The $\mathrm{PD}$ is formed by the tetramerization of S5 and S6 helices from each subunit/repeat (Figure 2D). A pore loop between S5 and S6 contains the selectivity filter (SF) that confers ion specificity. A linker helix between helices S4 and S5, termed the S4-S5 linker, connects the VSD to the PD (Bezanilla, 2005; Dehghani-Samani et al., 2019) (Figures 2A-C).

Given these similarities in channel topology and components, how is it that KCNQ1, hERG, and SCN5A perform such distinct functions, and produce phenotypically distinct forms of LQTS? To explore this question, a number of high-resolution ion channel structures, including cryo-electron microscopy (cryoEM) structures of frog and human KCNQ1 (Sun and Mackinnon, 2020) and of human hERG (Wang and Mackinnon, 2017), as well as the structures of the rat homolog of SCN5A (Jiang et al., 2020) and human $\mathrm{Na}_{\mathrm{v}} 1$ isoforms Nav1.4 and 1.7 (Pan et al., 2018; Shen et al., 2019; Xu et al., 2019), have been determined. Moreover, the frog KCNQ1 structure has been used to develop what is likely a reliable homology model for the human KCNQ1 channel in resting and fully active conformations (Kuenze et al., 2019). A homology model of human SCN5A in the resting state has also been devised (Kroncke et al., 2019). These structures and structural models reveal critical differences in the atomic details of KCNQ1, hERG, and SCN5A structures associated with their distinct functions and disease phenotypes. Notably, the subunits of KCNQ1 undergo domain swapping, with a similar arrangement observed in SCN5A but not in the hERG channel (Figure 2D). The monomeric sequence of SCN5A causes the channel to adopt an asymmetric three-dimensional fold, in contrast to the inherent symmetry of tetrameric hERG and KCNQ1 (Figure 2D). Additionally, the C-terminal domains contain distinct folds and mediate unique regulatory functions. These and other structural differences contribute to the varying properties of these three channels and to their distinct roles in the cardiac action potential.

The aim of this review is to compare and contrast the KCNQ1, hERG, and SCN5A channels using available structures and structural models as a guide. Through this lens, channel gating, regulation, LQTS mechanisms, and pharmacology will be discussed, in order to explore the molecular basis of these unique properties. 


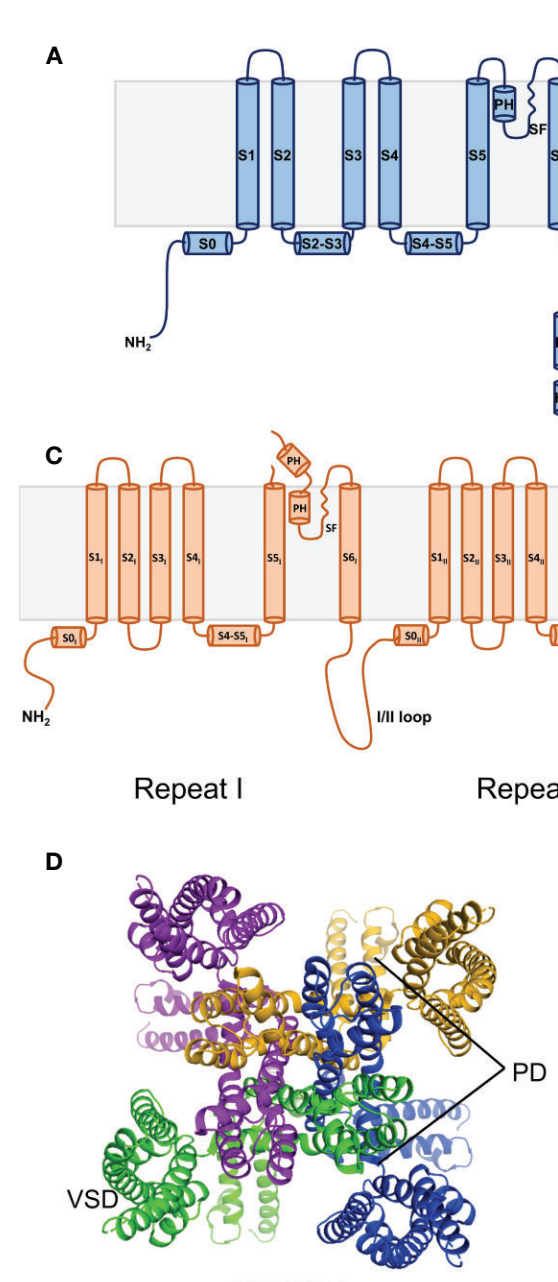

KCNQ1

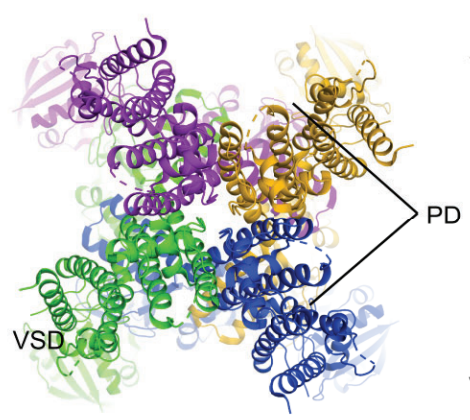

hERG

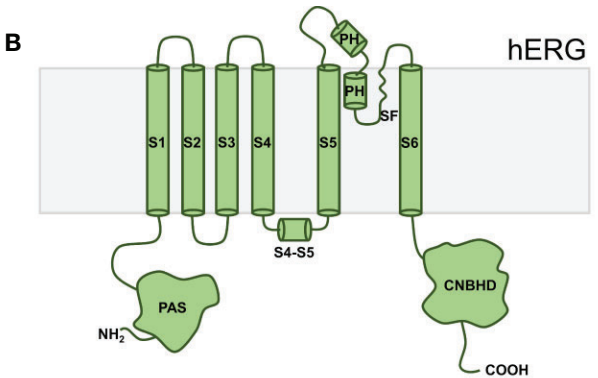

hERG

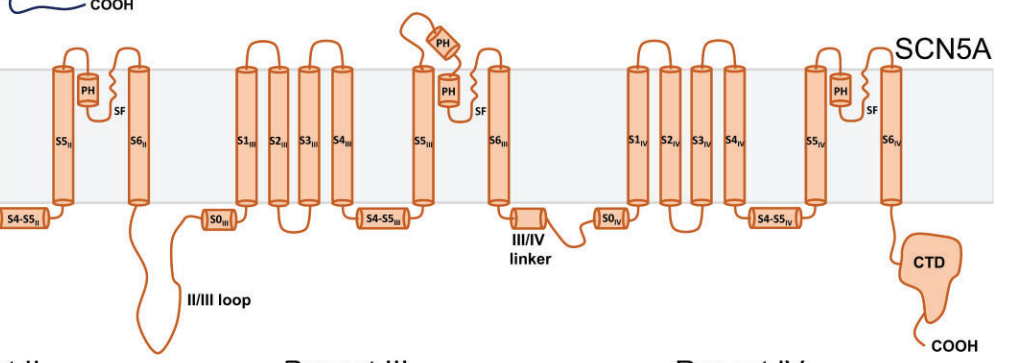

Repeat IV

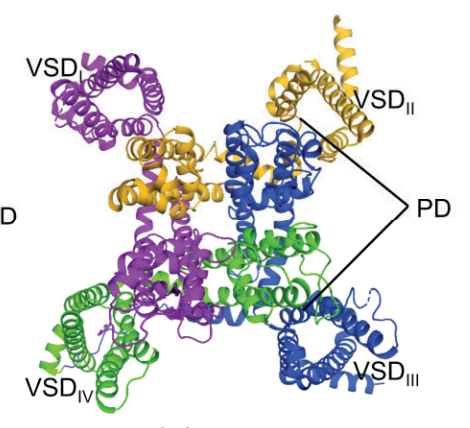

SCN5A

FIGURE 2 | Overall topology of KCNQ1, hERG, and SCN5A channels. (A) KCNQ1 topology. Transmembrane domain alpha helices are labeled S0-S6. PH indicates the pore helix. SF denotes the selectivity filter. S2-S3 indicates the S2-S3 linker. S4-S5 denotes the S4-S5 linker. Cytosolic alpha helices are labeled HA-HD. (B) hERG topology. PAS denotes the Per-ARNT-Sim domain. CNBHD indicates the C-terminal cyclic nucleotide-binding homology domain. (C) SCN5A topology. CTD indicates the C-terminal domain. (D) Top views of the human KCNQ1 (PDB ID: 6UZZ) (Sun and Mackinnon, 2020), human hERG (PDB ID: 5VA1) (Wang and Mackinnon, 2017), and rat SCN5A (PDB ID: 6UZ3) (Jiang et al., 2020) channels, respectively. Outer voltage-sensing domains (VSD) and the central pore domain (PD) are labeled.

\section{STRUCTURAL MECHANISMS OF CHANNEL GATING}

KCNQ1, hERG, and SCN5A undergo conformational changes in response to changes in membrane potential that result in channel opening or closing. These responses confer specific gating properties including activation, deactivation, inactivation, and recovery from inactivation (Hosseini, 2018; Zhang et al., 2018). In activation, protein conformational changes result in channel pore opening from a resting state, while deactivation entails a return to the resting state (Zhang et al., 2018). Inactivation confers a third channel state distinct from the activated and resting states which inhibits current flow prior to full deactivation (Zhang et al., 2018). While all three channels share common structural elements that are responsible for producing these states, there are also elements that give rise to specific gating properties in each channel, as discussed below.

\section{Activation and Ion Conduction KCNQ1}

Voltage-gated channels contain up to six positively-charged basic residues in the $\mathrm{S} 4$ helix, called gating charges, which move in the electric field of the membrane in response to voltage (Jiang et al., 2003). Gating charges are numbered according to their position in the S4 helix, from the extracellular to the intracellular side. In KCNQ1, S4 contains four arginine (R) gating charges that are conserved in other $\mathrm{K}_{\mathrm{V}}$ channels and confer voltage sensitivity. However, in KCNQ1 the canonical R3 is replaced by a neutral glutamine (Q234, Q3), and the fifth gating charge (K5 in Shaker) is replaced by histidine $(\mathrm{H} 241, \mathrm{H} 5)$, which in the membrane 
A

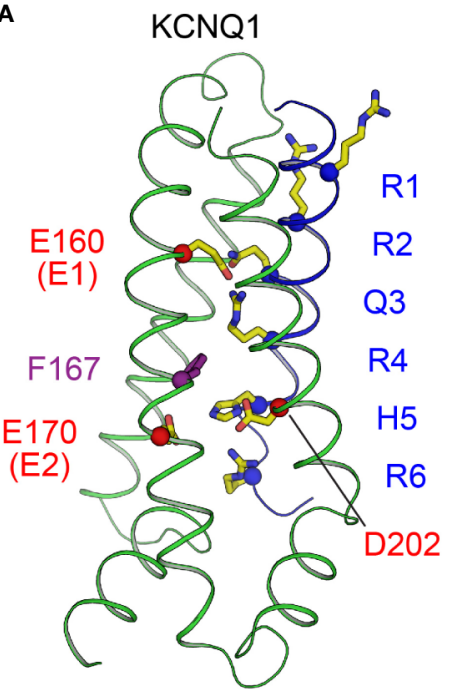

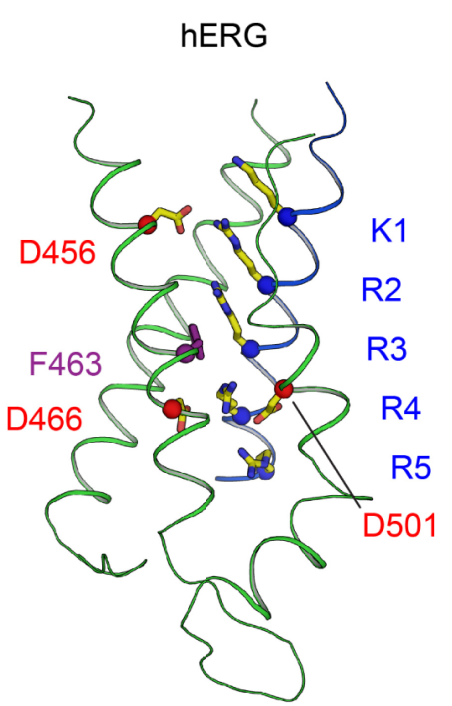

B

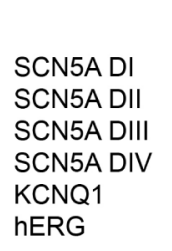

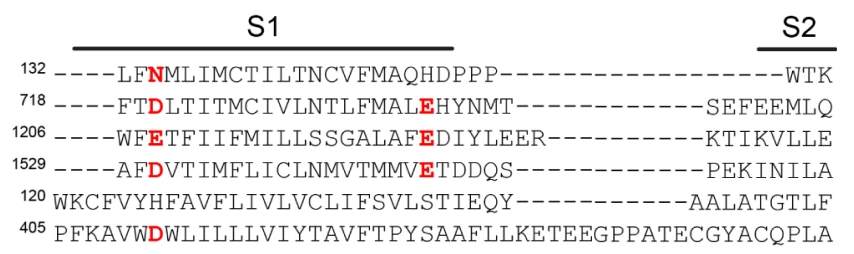

S2
SCN5A DI
SCN5A DII
SCN5A DIII
SCN5A DIV
KCNQ1
hERG

SCN5A DI
SCN5A DII
SCN5A DIII
SCN5A DIV
KCNQ1
hERG

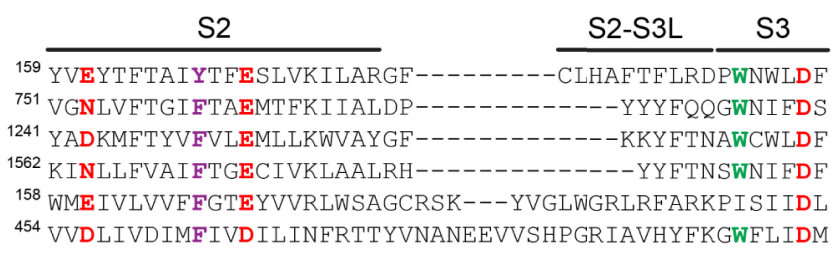

S3

$199 \overline{\text { SVI IMAYTTEFVDLGN--------VSALRTERVLRALKTISVISGLK }}$

787 I IVILSLMELGLSRMSN--------LSVLRSERLLRVFKLAKSWPTLN

1277 LIVDVSLVSLVANTLGF---AEMGP IKSLRTLRALRPLRALSRFEGMR

${ }^{1597}$ VVVILSIVGTVLSDI IQKYFF SPTLFRVIRLARIGRI LRL IRGAKGIR

${ }^{204}$ IVVVASMVVLCVGSKGQ--VFATSA IRGIRFLQILRMLHVDRQGGTWR

${ }^{503}$ VAAIP-FDLLIFGSGSE------ELIGLLKTARLIRLVRVARKLDRY-

FIGURE 3 | The voltage sensors of KCNQ1, hERG, and SCN5A. (A) Structure of the VSD from human KCNQ1 (left) (PDB: 6UZZ) (Sun and Mackinnon, 2020) and hERG (right) (PDB: 5VA2) (Wang and Mackinnon, 2017) in putative activated conformations. Basic residues on S4 (labeled in blue), acidic residues on S2 and S3 (labeled in red), and the phenylalanine residue in the gating charge transfer center (purple) are shown as sticks. The first four basic residues on S4 (R1-R4) in KCNQ1 and the first three (K1-R3) in hERG are located above the charge transfer center. (B) Multiple sequence alignment of VSDs I-IV of SCN5A with the VSDs of human KCNQ1 and hERG. Basic residues in S4 implicated with voltage sensing are colored blue, and acidic or polar residues in S1-S3 suggested to interact with S4 gating charges are colored red. The conserved aromatic residue at the gating charge transfer center in S2 is colored purple. Another tryptophan residue in S3, conserved in the VSDs of Nav channels and also present in hERG, is colored green.

environment is expected to be neutral at physiological $\mathrm{pH}$ (Figures 3A, B). Due to these substitutions at positions 3 and 5 , the S4 helix of KCNQ1 has a lower net positive charge $(+4)$ than Shaker-class $\mathrm{K}^{+}$channels, such as $\mathrm{K}_{\mathrm{V}} 1.2(+6)$. The lower net positive charge may explain why KCNQ1 S4 mutations that result charge loss or reversal (Panaghie and Abbott, 2007; Wu et al., 2010b) result in constitutive channel activity.

Voltage-gated channels also feature a charge transfer center (CTC), formed by a bulky aromatic ring and two negatively charged residues, that facilitates S4 movement (Tao et al., 2010). The KCNQ1 CTC consists of E170 (E2) and F167 on S2, and D202 on S3, which work with E160 (E1) to define the S4 position. During activation, S4 moves towards the extracellular side of the membrane (Nakajo and Kubo, 2007; Rocheleau and Kobertz, 2008; Osteen et al., 2010; Ruscic et al., 2013; Barro-Soria et al., 2014; Nakajo and Kubo, 2014; Barro-Soria et al., 2017) through interactions between basic gating charges and E1 and E2 in the CTC (Figure 3A). These interactions change during the course 
of activation, permitting S4 translocation. E1 interacts with R1 (R228) or R4 (R237) in the resting and activated states of the VSD, respectively (Wu et al., 2010a). S4 motion occurs in two distinct steps, transitioning through a stable intermediate before reaching the activated state (Wu et al., 2010a; Barro-Soria et al., 2014; Zaydman et al., 2014). The intermediate state features salt bridge interactions between E1 and R2, distinct from the resting and activated VSD states, according to the recently-determined structure of the intermediate state KCNQ1 VSD (Taylor et al., 2020). Interestingly, the pore of KCNQ1 opens in both the intermediate state (IO) and fully activated (AO) states (Zaydman et al., 2014). These two open states possess distinct channel properties with differing opening probabilities and pharmacology (Hou et al., 2017), and have distinct pore structures (Zaydman et al., 2014). Importantly, ion conductance when the VSD is in either the intermediate or activated state appears to be unique to KCNQ1. However, formation of the KCNQ1-KCNE1 complex eliminates the conductance associated with the VSD intermediate state (Zaydman et al., 2014), such that $\mathrm{I}_{\mathrm{Ks}}$ reflects only the fully activated state.

The ion conduction pathway in KCNQ1 is lined by the four S6 helices, with the SF on the extracellular side of the pore. Mutations in S6 cause changes in current amplitude and voltage dependence of activation (Wang et al., 1999; Seebohm et al., 2005; Panaghie et al., 2006; Hoosien et al., 2013). Comparison of S6 in the closed pore structure of human KCNQ1 (Sun and Mackinnon, 2020) with that of the open channel demonstrates that channel opening results from bending of S6 so that the cytosolic ends of the four S6 segments swing away from the central axis, enlarging the diameter of the pore to allow diffusion of $\mathrm{K}^{+}$into the central cavity. The hinge responsible for this bending motion in S6 is the P343-A344-G345 (PAG) motif, which corresponds to PVP in Shaker $\mathrm{K}^{+}$channels (Labro and
Snyders, 2012). Additionally, A336 may also be important in the motion of the activation gate, as mutations at this position alter the voltage dependence of activation (Seebohm et al., 2006).

Once the intracellular gate is opened, $\mathrm{K}^{+}$ions move through the pore along their electrochemical gradient. The backbone carbonyl oxygens of the TIGYG motif in the SF of KCNQ1 (TVGYG in $K_{V} 1.2$ and KcsA) (Figure 4A) and the sidechain of T312 form four evenly spaced $\mathrm{K}^{+}$binding sites (Figure 4B) that facilitate $\mathrm{K}^{+}$movement (Zhou et al., 2001). The arrangement of these oxygens mimics the displaced hydration shell of $\mathrm{K}^{+}$, which lowers the transfer energy from the aqueous cavity at the center of the channel to the SF, allowing conduction to occur at rates near the diffusion limit (Morais-Cabral et al., 2001; Zhou et al., 2001).

\section{hERG}

The cryo-EM structure of hERG shows an open pore and activated VSDs, with the first three gating charges (K1-R3) of S4 located on the extracellular side of the CTC (Figure 3A). This is a translocation of one charge fewer than in the activated VSD of KCNQ1 (Sun and Mackinnon, 2017) and Shaker-like $\mathrm{K}_{\mathrm{V}} 1.2-$ 2.1 (Long et al., 2007) where four gating charges are located above the conserved phenylalanine in the CTC. This observation agrees with gating current measurements suggesting a total charge movement of only $\sim 6$ elementary charge units (movement of 1.5 positive charges per S4 helix) for hERG during activation (Zhang et al., 2004), compared to 8 to 9 (2 positive charges per helix) for KCNQ1 (Ruscic et al., 2013) and 12 to 16 (3-4 positive charges per helix) for Shaker-like $\mathrm{K}_{\mathrm{V}}$ channels (Schoppa et al., 1992; Aggarwal and Mackinnon, 1996; Seoh et al., 1996). However, the ca. 50\% lower total gating charge movement for hERG relative to Shaker-like channels is not explained by differences in the number of S4 gating charges, since hERG has a total of five positively-charged residues on S4

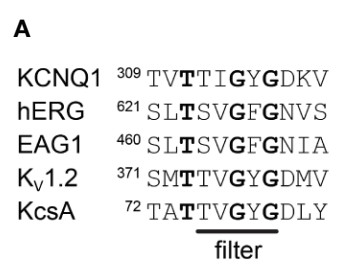

B

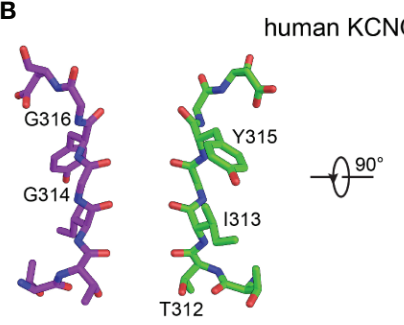

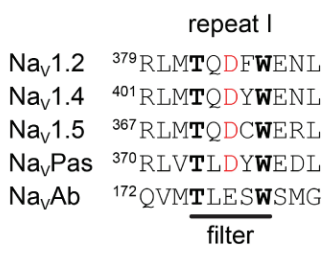
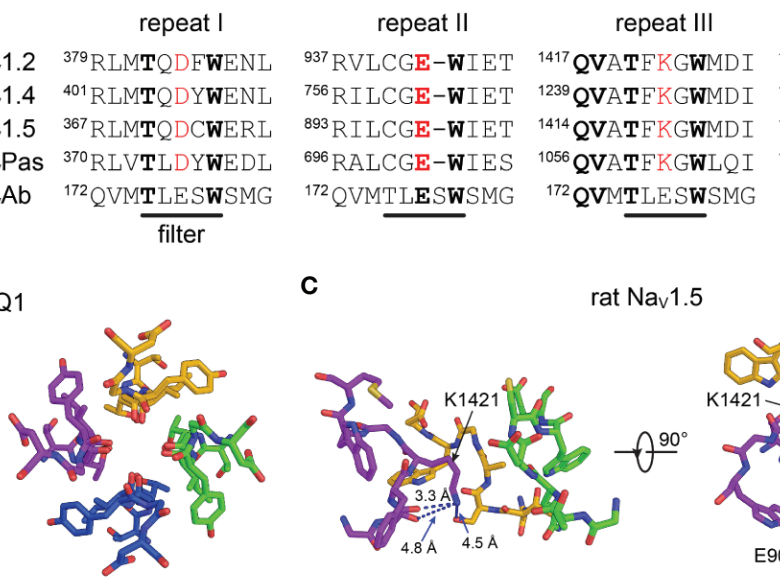

C

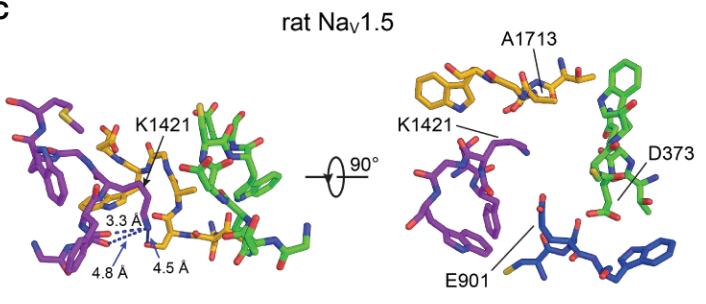

FIGURE 4 | The selectivity filters of $\mathrm{K}^{+}$and $\mathrm{Na}^{+}$channels. (A) Multiple sequence alignment of the SF region of selected $\mathrm{K}^{+}$(left) and $\mathrm{Na}^{+}$(right) channels. Conserved amino acids are highlighted in bold. Amino acids belonging to the DEKA signature motif in eukaryotic $\mathrm{Na}^{+}$channels are colored red. (B) Side and top view of the SF of human KCNQ1 (PDB: 6UZZ) (Sun and Mackinnon, 2020). Two subunits are omitted for clarity in the left plot. (C) Side and top view of the SF of SCN5A (PDB: 6UZ3) (Jiang et al., 2020). Repeat II is omitted for clarity in the left plot. 
and Shaker has six (Pless et al., 2011b). These combined structural and functional data thus imply that S4 translocates less in hERG during activation, resulting in smaller overall VSD conformational changes.

However, the activated VSD of hERG may not be fully defined. While the position of K1-R3 above the CTC in the cryo-EM structure of hERG is consistent with a depolarized VSD, some salt bridge interactions in the VSD have suboptimal geometry, particularly with $\mathrm{R} 4$. This is likely due to the limited resolution of the VSD in the final map (approximately 4.5-5.5 (Wang and Mackinnon, 2017), impeding unambiguous determination of sidechain conformations. While cryo-EM has proven to be a powerful structural tool, the resolution is often lower in the periphery of protein structures (Herzik et al., 2019). This can prevent accurate modeling of functional features and lead to discrepancies with experimental data. Molecular dynamics may be a be useful tool in refining cryo-EM structures to mitigate these discrepancies, as is currently being carried out for the hERG structure (Khan et al., 2020).

While a structure of hERG with a closed PD has not yet been determined, we can gain insight into the conformational changes that occur during pore opening using the closed-pore structure of the closely related rat potassium voltage-gated subfamily $\mathrm{H}$ member 1 channel (EAG1 or KCNH1) (Whicher and Mackinnon, 2016). In hERG, the intracellular gate is likely constricted by the Q664 side chains in S6, since the radius of the cavity at Q664 is almost $6 \AA$ in the open state hERG structure (Wang and Mackinnon, 2017), while at the corresponding position (Q476) in the EAG1 closed state structure, the pore is at its narrowest (<1 $\AA$ ) (Whicher and Mackinnon, 2016). Bending and displacement of the S6 helices is suggested by a glycine residue, located at the same position in both channels, acting as a gating hinge (G648 in hERG, G460 in EAG1).

The SF in hERG is unique among $\mathrm{K}_{\mathrm{v}}$ channels, containing a GFG motif (Figure 4A) in place of the typical GYG motif (Long et al., 2005). The position of the phenylalanine residue in this motif is different from the corresponding tyrosine in other $\mathrm{K}_{\mathrm{v}}$ channels of known structure (Wang and Mackinnon, 2017). This structural variation may have important implications for fast inactivation in hERG, as discussed below.

\section{SCN5A}

The mechanism of voltage sensing in $\mathrm{Na}_{\mathrm{V}}$ channels is thought to be similar to that of $\mathrm{K}_{\mathrm{V}}$ channels. The $\mathrm{S} 4$ helix is the key sensor of transmembrane voltage. Pairing of the positively-charged residues in S4 with polar or negatively-charged residues catalyzes S4 movement from its inward resting-state position to the outward activated state upon membrane depolarization. Recent cryo-EM structures of $\mathrm{Na}_{\mathrm{V}}$ channels with VSDs in activated (Yan et al., 2017b; Pan et al., 2018; Pan et al., 2019; Shen et al., 2019; Jiang et al., 2020) and resting (Clairfeuille et al., 2019; Wisedchaisri et al., 2019; Xu et al., 2019) conformations uncover a remarkable 10 to $15 \AA$ translation of S4 across the membrane, fully consistent with the "sliding helix" model of VSD activation (Catterall, 1986). A conserved aromatic residue (tyrosine in repeat I, phenylalanine II, III, and IV) on S2 serves as the hydrophobic plug that constricts the S4 gating canal and prevents ion leak through the VSD (Jiang et al., 2020). This hydrophobic plug is mechanistically identical to the corresponding residues in the CTC of $\mathrm{K}_{\mathrm{V}}$ channels (F167 in KCNQ1, F463 in hERG). However, in contrast to the VSD of KCNQ1 and hERG, the number of basic residues on S4 in SCN5A varies from four (repeat I) to six (repeat IV) (Jiang et al., 2020) (Figure 3B). The variation in the number of gating charges and the heterogenous distribution of acidic and polar residues on S1-S3 between KCNQ1, hERG, and SCN5A (Figure 3B) may be responsible for their distinct voltage sensitivities and kinetics of VSD activation. In $\mathrm{Na}_{\mathrm{V}}$ channels, the VSDs of repeats I, II, and III are mainly responsible for channel activation and pore opening, while the VSD of repeat IV is responsible for initiating and maintaining fast inactivation (Chanda and Bezanilla, 2002; Capes et al., 2013; Clairfeuille et al., 2019). This activation process has been studied in detail for the human skeletal muscle channel $\mathrm{Na}_{\mathrm{V}} 1.4$, giving rise to the "asynchronous gating model" (Chanda and Bezanilla, 2002; Capes et al., 2013; Goldschen-Ohm et al., 2013) wherein the S4 segments of repeats I, II, and III move quickly, permitting conductance before activation of $\mathrm{VSD}_{\mathrm{IV}}$, while $\mathrm{S} 4$ movement in repeat IV is slower and represents the rate-limiting step for development of and recovery from inactivation.

The structure and function of the selectivity filter in SCN5A and other $\mathrm{Na}_{\mathrm{V}}$ channels differs fundamentally from that of $\mathrm{K}_{\mathrm{V}}$ channels such as KCNQ1 and hERG (Figure 4). The SF gate in $\mathrm{Na}_{\mathrm{V}}$ channels is wider to allow $\mathrm{Na}^{+}$ions to pass in a partially hydrated state (Hille, 1971; Naylor et al., 2016). The extracellular vestibule of $\mathrm{Na}_{\mathrm{V}}$ channels is lined by negatively-charged residues that recruit $\mathrm{Na}^{+}$ions to the SF. Coordination by both sidechain and backbone carbonyls contribute to the $\mathrm{Na}^{+}$permeation mechanism (Chakrabarti et al., 2013; Ulmschneider et al., 2013; Naylor et al., 2016). Additionally, the SF of eukaryotic $\mathrm{Na}_{V}$ channels is formed in a pseudo-symmetric fashion by four short helix-connecting turn motifs from each subunit (Figure 4A). Four distinct residues-DEKA, one in each repeat-form the signature motif for $\mathrm{Na}^{+}$selectivity found in all human $\mathrm{Na}_{V}$ channel pore-forming repeats (Figure 4C). The lysine residue in the DEKA motif (K1419 in SCN5A) confers selectivity for $\mathrm{Na}^{+}$ and prevents permeability of $\mathrm{Ca}^{2+}$ (Favre et al., 1996). The recent rat SCN5A structure suggests a mechanism by which K1419 contributes to this selectivity of $\mathrm{Na}^{+}$over $\mathrm{Ca}^{2+}$, whrein lysine forms a charge delocalization network at a constriction point in the SF. Only $\mathrm{Na}^{+}$ions, which have a compatible size and electric field strength, are able to pass through (Jiang et al., 2020).

\section{Electromechanical Coupling KCNQ1}

In the absence of accessory subunits, KCNQ1 exhibits a constitutive current reflecting close-to-open state transitions even at very negative $(\sim-120 \mathrm{mV})$ voltages (Ma et al., 2011$)$. Analysis of a large group of KCNQ1 mutants suggests that gating follows an allosteric model (Ma et al., 2011). According to this model, the pore can open independently of the state of the VSD, but VSD activation increases the probability of pore opening. 


\section{A}
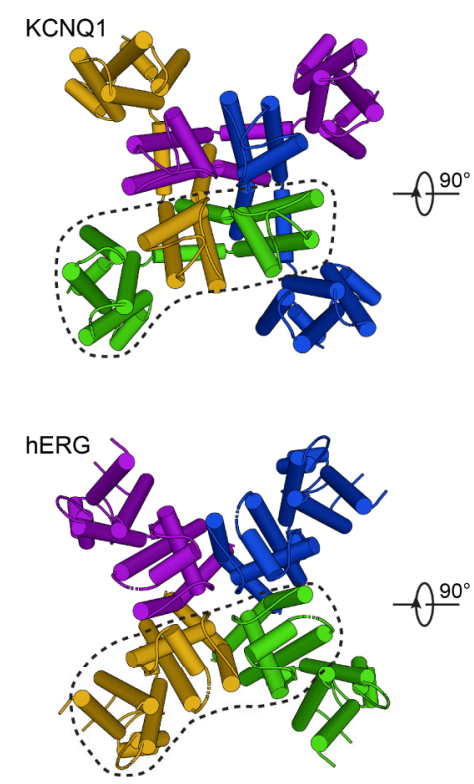

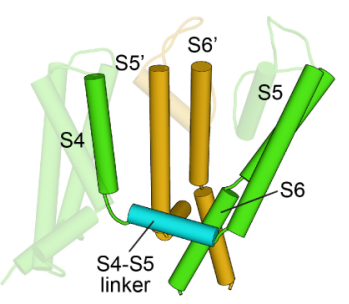

\section{B}
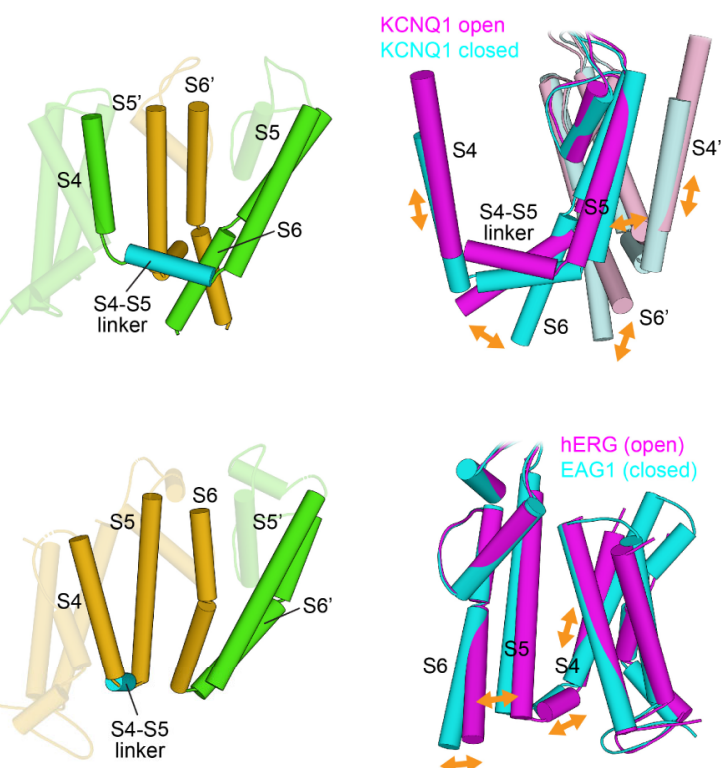

FIGURE 5 | Structural features of electromechanical coupling in the KCNQ1 and hERG channels. (A) Left: Extracellular view of KCNQ1 and hERG transmembrane segments S0 to S6. Individual subunits are drawn with different colors. KCNQ1 (PDB: 6UZZ) (Sun and Mackinnon, 2020) has a domain-swapped architecture whereas hERG (PDB: 5VA1) (Wang and Mackinnon, 2017) channels are non-domain-swapped. Right: Cartoon representation of a single subunit and its neighboring pore segments (S5 to S6). In KCNQ1, the VSD and PD are bridged by an extended $\alpha$-helical S4-S5 linker, whereas in hERG, S4 and S5 are connected by only a short helix. (B) Implications for the direction of coupled motions between the VSD and PD based on molecular modeling of KCNQ1 in open and closed conformations (top) and comparison of the open state structure of hERG with the closed state structure of EAG1 (PDB: 5K7L) (Whicher and Mackinnon, 2016) (bottom). In the domain-swapped KCNQ1 channel, the S4 movement is transmitted to the gate through the S4-S5 linker. In hERG and EAG1, the S4 movement is proposed to exert a direct force on the S5-S6 interface to compress or open the channel gate.

Furthermore, KCNQ1 opening does not require concerted VSD movements (Osteen et al., 2012). Indeed, the VSDs appear to move independently, and pore opening can occur before all VSDs are activated (Osteen et al., 2012), consistent with an allosteric gating model.

Allosteric coupling for KCNQ1 is thought to be mediated by interactions between the VSD and the PD that translate S4 movement to channel opening and closing (Figure 5). Studies of KCNQ1 (Boulet et al., 2007; Labro et al., 2011) and of other $\mathrm{K}_{\mathrm{V}}$ channels (Lu et al., 2001; Lu et al., 2002; Long et al., 2005) have pointed to the interface between the S4-S5 linker and the Cterminal end of $\mathrm{S} 6\left(\mathrm{~S} 6_{\mathrm{C}}\right)$ as one important mediator of electromechanical coupling (Figure 5A). Certain mutations in the S4-S5 linker (Labro et al., 2011) and S6 ${ }_{C}$ (Boulet et al., 2007) slow the opening rate and shift channel activation to more depolarized voltages, while other mutations, specifically at V254 in the S4-S5 linker and at L353 in S6 C $_{\text {, promote a }}$ constitutively open channel. Interestingly, a V254L/L353A double mutant rescued channel closing, suggesting that the S4S5 linker interacts with $S 6_{C}$ to stabilize the closed state. Relocation of the S4-S5 linker during gating abolishes this interaction, releasing tension on S6 and allowing it to kink at the PAG gating hinge in a cantilever-like fashion to promote channel opening (Figure 5B). This coupling mechanism is intrinsically weak for KCNQ1, requiring modulation by auxiliary molecules (see below). Recent work has further elucidated the molecular details of S4-S5 linker relocation, revealing a two-stage mechanism involving alternative binding modes of the S4-S5 linker to the PD (Hou et al., 2020). This twostage mechanism may apply to the majority of domain-swapped $\mathrm{K}_{\mathrm{v}}$ channels.

\section{hERG}

The relative positioning of the VSD and PD in hERG (Wang and Mackinnon, 2017) (Figure 5A) and the EAG1 channel (Whicher and Mackinnon, 2016) suggests that the mechanism by which movements in the VSD are transduced to pore opening is different from other $\mathrm{K}_{\mathrm{V}}$ channels (Toombes and Swartz, 2016; Barros et al., 2019). This notion is supported by the finding that cutting the S4-S5 linker in hERG through separate expression of the VSD and PD fails to significantly perturb activation kinetics (Lorinczi et al., 2015). In contrast to the lever mechanism proposed for KCNQ1, lateral S4 movement in hERG toward the pore could both alter S4-S5 linker/S6 interactions and exert force through the S4-S5 linker directly onto S5. Displacement of S5 may be transmitted through the S5-S6 interface for opening or closing of the cytosolic gate (Wang and Mackinnon, 2017) (Figure 5B). A structure of hERG in the resting state may provide further insight into the molecular features of this distinct coupling mechanism. 


\section{SCN5A}

Much of our knowledge about the coupling in SCN5A originates from studies of ancestral $\mathrm{Na}_{V}$ channels. Comparison of locked resting state structures of the bacterial sodium channel $\mathrm{Na}_{\mathrm{V}} \mathrm{Ab}$ (Wisedchaisri et al., 2019) and of the chimeric human $\mathrm{Na}_{\mathrm{V}} 1.7-$ $\mathrm{VSD}_{\mathrm{II}}-\mathrm{Na}_{\mathrm{V}} \mathrm{Ab}$ channel (Xu et al., 2019) with activated state structures (Yan et al., 2017b; Pan et al., 2018; Pan et al., 2019; Shen et al., 2019; Jiang et al., 2020) provide new insight into the mechanism of electromechanical coupling in $\mathrm{Na}_{\mathrm{V}}$ channels. The S4-S5 linker appears to undergo movement similar to that of KCNQ1. The linker constrains the S5 and S6 helices in the resting/closed state, with looser interactions in the activated/ open state. Coupling between the VSD and PD during pore opening must involve loosening of linker/PD interactions as S4 moves outward as in KCNQ1, albeit with tighter coupling of S4 and S4-S5 linker movement. The direct connection between S4 movement and pore opening may be crucial for rapid activation of sodium channels.

Comparison of the cockroach $\mathrm{Na}_{\mathrm{V}} \mathrm{PaS}$ channel structure (Shen et al., 2017), featuring a closed pore and VSDs in distinct activation states, to other eukaryotic $\mathrm{Na}_{V}$ channel structures suggests that additional structural shifts may be at work in eukaryotic $\mathrm{Na}_{\mathrm{V}}$ channels to couple VSD to PD movement. Moreover, the distinct sequence of repeats I to IV (including that of the four S4-S5 linkers) and asynchronous voltage sensor movement in eukaryotic $\mathrm{Na}_{\mathrm{V}}$ channels (Chanda and Bezanilla, 2002) suggest that distinct interactions couple the VSD of each repeat to the PD. Electromechanical coupling mechanisms may thus be more complex in SCN5A.

\section{Inactivation KCNQ1}

Inactivation in KCNQ1 follows a mechanism distinct from canonical mechanisms (Hou et al., 2017). In the absence of KCNE1, KCNQ1 only partially inactivates, in a manner dependent on the IO and AO open states (Pusch et al., 1998). Differences in VSD-PD coupling between these two states appear to contribute to inactivation. The AO state has a lower coupling efficiency than IO, producing a lower open probability. Transition from IO to AO thus results in partial inactivation because the channel is open but less conductive (Hou et al., 2017). However, association with the KCNE1 accessory $\beta$ subunit removes KCNQ1 inactivation (Pusch et al., 1998; Seebohm et al., 2003c), making inactivation irrelevant to cardiac KCNQ1 function.

\section{hERG}

Inactivation plays a critical role in hERG activity in the action potential. Entry into and out of inactivation is both fast and voltage-dependent, properties that maintain the plateau of the action potential (Perry et al., 2015). Additionally, the voltage dependence of inactivation appears to be independent of that of activation (Vandenberg et al., 2006; Cheng and Claydon, 2012), indicating that activation and inactivation may operate through distinct mechanisms. Inactivation in hERG is C-type (Smith et al., 1996), occurring through structural changes in the SF
(Herzberg et al., 1998 14243; Hoshi and Armstrong, 2013). While the structural basis of C-type inactivation is not completely understood, work on hERG and other $\mathrm{K}^{+}$channels has provided useful insight. Several residues in the KcsA SF form stabilizing hydrogen bond networks that suppress inactivation (Doyle et al., 1998; Bhate et al., 2010; Cuello et al., 2010; Vandenberg et al., 2012). While these residues are conserved in many $\mathrm{K}_{\mathrm{v}}$ channels (Whicher and Mackinnon, 2016), they are not present in hERG. This implies that the hERG SF is more liable to collapse, leading to inactivation (Fan et al., 1999; Vandenberg et al., 2012). Indeed, in molecular dynamics simulations, several residues in the hERG SF shift in and out of the pore axis, particularly F627 in the GFG motif (Stansfeld et al., 2008). In the hERG cryo-EM structure, the orientation of F627 is offset compared to other $\mathrm{K}_{\mathrm{v}}$ channels (Whicher and Mackinnon, 2016), and a hERG S631A mutant, which has an F627 sidechain orientation similar to other structures, does not inactivate (Wang and Mackinnon, 2017). Additionally, mutation of T432 and A443 (corresponding to S620 and S631 in hERG) in non-inactivating EAG1 to serine was sufficient to impart inactivation behavior (Ficker et al., 2001), perhaps due to reorientation of the SF to match that of hERG. These results indicate that the unique positioning of F627 is critical for hERG fast inactivation behavior. C-type inactivation may involve other rearrangements (Loots and Isacoff, 1998), including coupling the SF to motions in S1, S5, and S6 (Ferrer et al., 2011; Wang et al., 2011; Perry et al., 2013a; Perry et al., 2013b).

\section{SCN5A}

Fast inactivation in SCN5A halts inward $\mathrm{Na}^{+}$current triggered by cardiac depolarization, permitting subsequent outward currents (e.g. $\mathrm{I}_{\mathrm{Ks}}, \mathrm{I}_{\mathrm{Kr}}$ ) to repolarize the cell in preparation for the next action potential (Ghovanloo et al., 2016) (Figure 6A). The S4 segment in $\mathrm{VSD}_{\text {IV }}\left(\mathrm{S}_{\text {IV }}\right)$ is critical for fast inactivation, along with the IFM motif in the III/IV linker (Ahern et al., 2016; Ghovanloo et al., 2016) (Figures 6B, C). Roles of individual basic sites of $\mathrm{S}_{\mathrm{IV}}$ in $\mathrm{Na}_{\mathrm{v}}$ inactivation have been elucidated, with mutations in R1 and R2 delaying inactivation onset and mutations in R3 and R4 delaying recovery from inactivation (Nakajima et al., 2019). Furthermore, structures of an engineered human $\mathrm{Na}_{\mathrm{V}} 1.7-\mathrm{Na}_{\mathrm{V}} \mathrm{PaS}$ channel with $\mathrm{VSD}_{\mathrm{IV}}$ trapped in a resting state (Clairfeuille et al., 2019) along with the structures of $\mathrm{Na}_{\mathrm{v}} \mathrm{PaS}$ (Shen et al., 2017), electric eel (Yan et al., 2017b) and human $\mathrm{Na}_{\mathrm{v}} 1.4$ channels (Pan et al., 2018) have provided new structural insight into the mechanism of fast inactivation in $\mathrm{Na}_{\mathrm{V}}$ channels (Figure 6D). In the resting state $\mathrm{R} 5$ on $\mathrm{S} 4$ IV forms an electrostatic bridge with the $\alpha 1$ helix of the C-terminal cytoplasmic domain (CTD), together with K7 and R8 on the S4-S5 linker. The CTD in turn binds the III/IV linker, sequestering the IFM motif (Figure 6D, left plot). $\mathrm{VSD}_{\mathrm{IV}}$ activation releases the connection between the CTD and the III/IV linker (middle and right plots in Figure 6D), permitting the IFM motif to bind to a hydrophobic pocket formed by the S4-S5 linkers and S6 helices of repeats III and IV, along with $\mathrm{S}_{\mathrm{IV}}$ (Figures 6C, E). The insertion of the IFM motif into this pocket causes a twisting in the S6 helices that closes the gate (Yan et al., 2017b; Pan et al., 2018). 


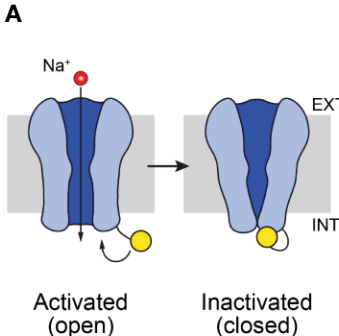

(open)
B

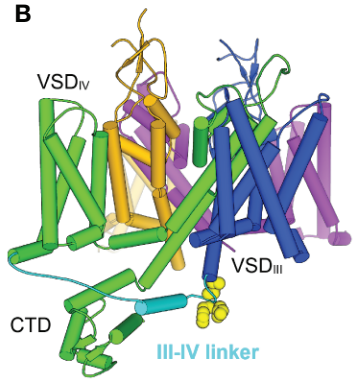

human Nav1.5 (Model)
C

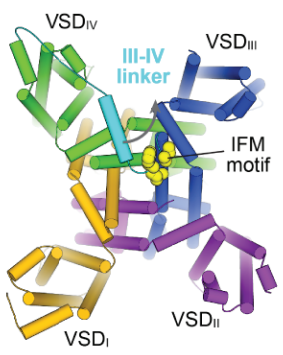

human Nav1.5 (Model) inactivation particle unbound

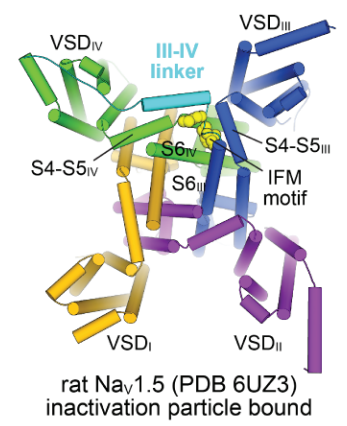

inactivation particle bound
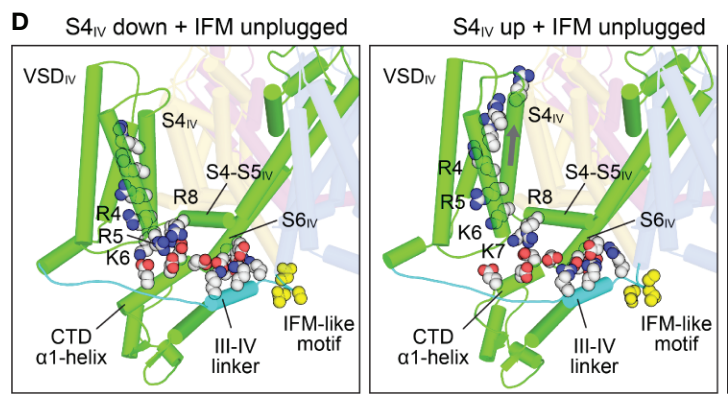

S4IV UP + IFM plugged in $E$
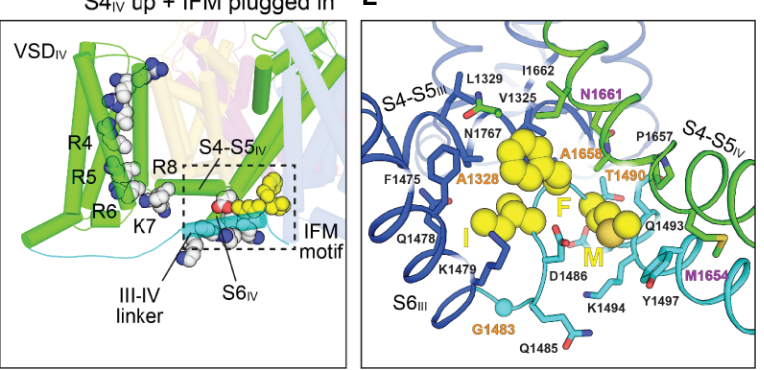

FIGURE 6 | Structural mechanisms of Nav channel inactivation. (A) Nav channels transition from an activated open state to a non-conducting inactivated state after depolarization. Inactivation is induced by binding of a C-terminal motif (yellow) to the cytosolic side of the channel leading to pore closure. The cell membrane is indicated with a gray rectangle, with the extracellular (EXT) and intracellular space (INT) labeled. (B) Closed state model of SCN5A (Kroncke et al., 2019) highlighting structural elements involved in fast inactivation. The III-IV linker (cyan) connecting S6 III (blue) with VSD IV (green) contains the IFM motif (yellow spheres) which is the key structural element responsible for inactivation. The position of the III-IV linker is constrained by the CTD following S6 $\mathrm{IV}_{\mathrm{V}}$ (C) Comparison of the SCN5A model in (B) with a cryo-EM structural model of rat SCN5A (PDB: 6UZ3)(Jiang et al., 2020) in a putative inactivated state viewed from the intracellular side. The III-IV linker undergoes a large shift in the SCN5A structure and the IFM motif is docked into a pocket surrounded by the S4-S5 linkers and S6 helices of repeats III and IV. (D) Structural states and transitions proposed to be involved in fast inactivation in Nav channels. Left: Structure of a chimeric Nav1.7-NavPaS channel (Clairfeuille et al., 2019) (PDB: 6NT3) with S4IV in a "down" position and an IFM-like motif unbound. Basic sites R5-R8 bridge to conserved acidic residues on the CTD which facilitates binding of the III-IV linker to S6 IV. Middle: Structure of a chimeric Nav1.7-NavPaS channel (Clairfeuille et al., 2019) (PDB: 6NT4) with S4IV in a 'up' position and an IFM-like motif unbound. The electrostatic bridge between S4 IV and the CTD is broken possibly increasing the positional dynamics of the CTD and III-IV linker. Right: Structure of SCN5A (PDB: 6UZ3) (Jiang et al., 2020) with S4IV in an 'up' position and the IFM motif plugged into the cytosolic cavity between repeats III and

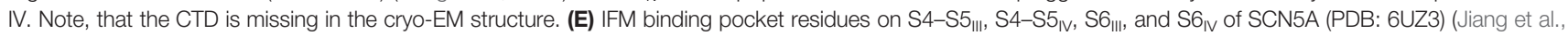
2020). The S6 $\mathrm{IV}$ helix backbone in the front is not shown for clarity. Residues that are important for fast inactivation and those found in various types of myotonia (Pan et al., 2018) are labeled magenta and orange, respectively.

\section{REGULATION OF CHANNEL GATING BY INTRACELLULAR DOMAINS AND AUXILIARY MOLECULES}

The function of these cardiac channels is tightly regulated to produce currents that faithfully give rise to the cardiac action potential under a host of physiological conditions and prevent early or delayed contractility. We focus here on regulation through the channel cytoplasmic domains as well as regulation by auxiliary (beta) subunits and lipids. Channels are regulated through other mechanisms such as phosphorylation, but are not covered in this review.

\section{Regulation Involving Cytoplasmic Domains KCNQ1}

The C-terminal cytoplasmic domain (CTD) of KCNQ1 contains four alpha helices in lieu of the T1 tetramerization domain common to $\mathrm{K}_{\mathrm{v}}$ channels outside the KCNQ family (Haitin and Attali, 2008). The proximal HA and HB helices form an antiparallel bundle with an IQ motif in HA and a 1-5-10 motif in $\mathrm{HB}$ that together enable calmodulin (CaM) binding (YusNajera et al., 2002). When bound, CaM prevents channel inactivation in KCNQ1, but inhibits the opening of other KCNQ family members (KCNQ2-KCNQ5) (Chang et al., 2018). While the N-lobe of CaM may be constitutively bound to both calcium and the proximal HA and HB helices (Ghosh et al., 2006; Bernardo-Seisdedos et al., 2018), the C-lobe of CaM binds calcium only at higher concentrations (BernardoSeisdedos et al., 2018). Calcium binding by the C-lobe induces a conformational change in $\mathrm{CaM}$, facilitating KCNQ1 opening (Bernardo-Seisdedos et al., 2018; Chang et al., 2018). The C-lobe interacts with HA and with a loop in the S2-S3 linker (Sun and Mackinnon, 2017). This feature may contribute to the unique mode of CaM modulation of KCNQ1.

The distal helices HC and HD of KCNQ1 form a selfassembling intersubunit coiled-coil motif that promotes channel tetramerization (Yus-Najera et al., 2002; Howard et al., 2007; Sun and Mackinnon, 2017). The distal coiled-coil domain 
is not only essential for channel tetramerization, but also for subunit specificity, permitting the exclusive formation of KCNQ1 homotetramers (Schwake et al., 2003; Haitin and Attali, 2008; Sachyani et al., 2014). In contrast, other KCNQ family proteins form heteromers (Schwake et al., 2003). Swapping the coiled-coil domain of KCNQ1 with that of KCNQ3 allows the resulting chimera to co-assemble with other KCNQ isoforms and generate channels with altered gating properties (Schwake et al., 2003; Schwake et al., 2006). Residues conferring specific KCNQ1 homotetramer formation have been identified in the HD helix (Schwake et al., 2006; Wiener et al., 2008). Key interactions involve both the hydrophobic core of the assembly and exterior electrostatic interactions, with a number of the residues involved subject to LQTS-associated mutations (Howard et al., 2007; Wiener et al., 2008).

\section{hERG}

hERG channels contain an N-terminal Per-ARNT-Sim (PAS) domain, which is also present in the N-terminus of many signaling proteins (Henry and Crosson, 2011). An additional Cterminal cyclic nucleotide-binding homology domain (CNBHD) similar to cytoplasmic domains in hyperpolarization-sensitive cyclic nucleotide-gated channels is present, but without ligandbinding properties (Codding and Trudeau, 2019). The PAS and the CNBHD appear to be critical for modulating the kinetics of slow deactivation in hERG (Gustina and Trudeau, 2011). Furthermore, slow deactivation is dependent on the direct interaction of these domains, particularly between R56 of the PAS and D803 of the CNBHD and between N12 of the N-terminal Cap (N-Cap) and E788 of the CNBHD (Ng et al., 2014; Kume et al., 2018). Interactions between the N-Cap and PAS domains with the C-linker also appear to be important for deactivation (Gustina and Trudeau, 2011; Ng et al., 2014), and the extreme Nterminus is likely essential, possibly interacting with the VSD through a patch of positively-charged residues in the N-Cap tail (Muskett et al., 2011). Indeed, the hERG cryo-EM structure corroborates the positioning of the $\mathrm{N}$-Cap tail relative to the VSD (Wang and Mackinnon, 2017). The N-Cap tail may even be constitutively bound to the VSD (Morais Cabral et al., 1998; De La Pena et al., 2011), implying that slow deactivation could involve movement of the PAS toward the plasma membrane to alter this interaction (Barros et al., 2018).

\section{SCN5A}

Multiple structures of the SCN5A CTD in complex with regulatory factors (Wang et al., 2012; Gabelli et al., 2014; Gardill et al., 2019) have been determined, helping to elucidate how CaM (Johnson et al., 2018) and fibroblast growth factorhomologous factors (FHFs), specifically FGF13, modulate inactivation (Liu et al., 2003; Wang et al., 2012; Musa et al., 2015; Yang et al., 2016). CaM binds the CTD in a calciumdependent manner (Kim et al., 2004; Hovey et al., 2017; Johnson et al., 2018; Urrutia et al., 2019), altering inactivation kinetics (Yan et al., 2017a; Johnson et al., 2018) and promoting recovery from inactivation (Johnson et al., 2018). In the absence of FHF, the C-lobe of apo-CaM binds to the IQ motif located in an extended helix on the CTD, while the N-lobe binds to the preceding EF hand-like domain (EFL) (Gabelli et al., 2014). With increasing intracellular calcium, holo-CaM has stronger affinity for the SCN5A III/IV linker (Johnson et al., 2018) and the N-lobe disassociates from the EFL (Gardill et al., 2019). These calcium-dependent interactions with the III/IV linker may facilitate CaM modulation of inactivation (Hovey et al., 2017; Johnson et al., 2018). FGF13 slows both inactivation onset and inactivation recovery in SCN5A (Yang et al., 2016), opposing CaM modulation. Importantly, binding of FGF13 to the SCN5A EFL (Wang et al., 2012) prevents binding of the apo-CaM N-lobe to the same domain (Wang et al., 2012). The EFL itself also adopts different orientations when bound to FGF13 (Wang et al., 2012) or CaM (Gabelli et al., 2014). Because the EFL also binds to the III/IV linker containing the IFM inactivation motif (Shen et al., 2017; Clairfeuille et al., 2019), EFL conformational changes upon binding of CaM or FGF13 may contribute to inactivation modulation by these proteins.

SCN5A also contains two cytoplasmic loops linking repeats I and II and repeats II and III, respectively. Unlike the III/IV linker, these loops are around 200 residues long (Pan et al., 2018) and likely disordered, based on the absence of these loops in cryo-EM structures. However, important regulatory events such as phosphorylation (Marionneau et al., 2012; Iqbal et al., 2018) and cofactor binding (Wu et al., 2008) that modulate channel properties have been identified in these loops.

\section{Auxiliary Beta Subunits KCNQ1}

KCNQ1 co-assembles with a family of single span membrane proteins (KCNE1-5) (Abbott, 2014), and this interaction appears to be tissue-specific. In the heart, KCNQ1 is complexed with KCNE1, and the KCNQ1-KCNE1 channel exhibits greater single-channel conductance, opening at more positive potentials, and delayed activation compared to KCNQ1 alone (Barhanin et al., 1996; Sanguinetti et al., 1996). This heteromultimeric channel also exhibits altered $\mathrm{Rb}^{+} / \mathrm{K}^{+}$ selectivity (Pusch et al., 2000) and loss of inactivation (Pusch et al., 1998; Pusch et al., 2000). These properties are essential for the generation of the slow delayed rectifier current $\left(\mathrm{I}_{\mathrm{Ks}}\right)$ in repolarizing cardiomyocytes. In gastric parietal cells, KCNQ1 co-assembles with KCNE2 to create constitutive $\mathrm{K}^{+}$currents essential for gastric acid secretion (Heitzmann et al., 2004; Roepke et al., 2006). In intestinal epithelial cells KCNQ1 complexes with $\mathrm{KCNE} 3$ to allow $\mathrm{K}^{+}$recycling for transepithelial chloride ion secretion (Preston et al., 2010). In contrast to KCNE1, KCNE2 and KCNE3 render KCNQ1 constitutively open (Schroeder et al., 2000; Tinel et al., 2000).

Due to the critical role of the KCNQ1-KCNE1 channel in the cardiac action potential, many research groups have probed the structural interaction between these proteins (Tapper and George, 2001; Xu et al., 2008; Chung et al., 2009; StrutzSeebohm et al., 2011; Wang et al., 2011; Chan et al., 2012; Li et al., 2014). Combined results indicate that the transmembrane helix of KCNE1 binds in the cleft between neighboring KCNQ1 


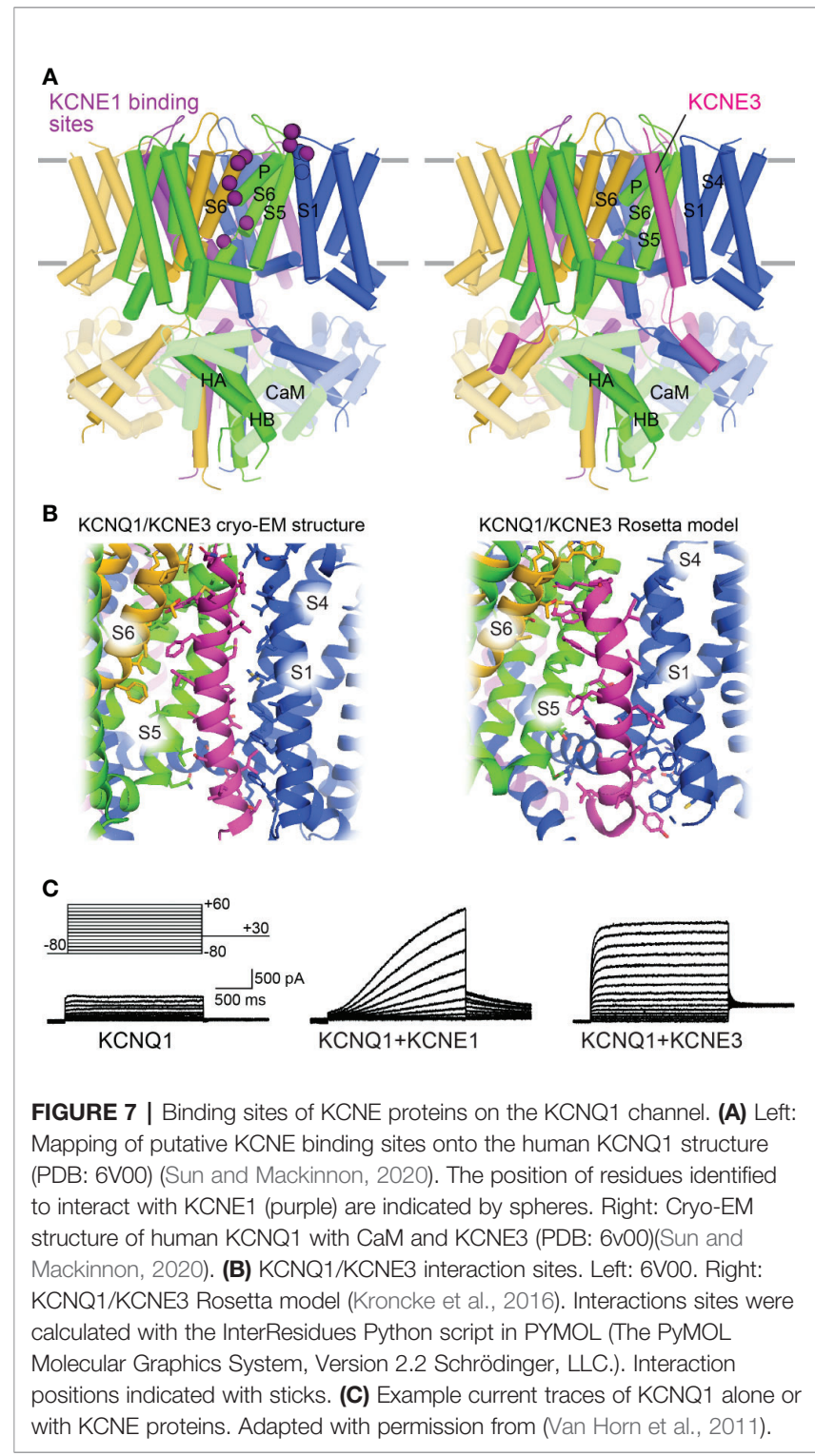

subunits and interacts with both the VSD and PD. These binding sites overlap the binding cleft of KCNE3 (Sun and Mackinnon, 2020), indicating that KCNE1 may bind to the same site (Figure 7A). In the human KCNQ1-KCNE3 cryo-EM structure (Sun and Mackinnon, 2020) and an earlier Rosetta model of the complex (Kroncke et al., 2016), this cleft is formed by three KCNQ1 subunits (Figure 7B). KCNE3 contacts the cytoplasmic half of S5 in one subunit, the extracellular side of S6 in a second one, and along the entire length of S1 and the cytoplasmic side of S4 in a third subunit. Importantly, KCNE1 and KCNE3 produce KCNQ1 currents with different voltage dependencies and gating kinetics (Figure 7C). Given the possibility of a common binding cleft, differential modulation of KCNQ1 by KCNE1 and KCNE3 may involve different sidechain interactions within this cleft.

Mutations in the KCNE1 transmembrane helix, particularly at F57, T58, and L59 (Melman et al., 2001; Melman et al., 2002), alter the ability of this subunit to modulate the function of KCNQ1. The effect of this 'activation' triplet on KCNQ1 gating is altered by mutations at residues S338, F339, F340, and A341 in S6 (Melman et al., 2004; Panaghie et al., 2006; Strutz-Seebohm et al., 2011; Li et al., 2014), suggesting a functional link between these residues. However, modeling studies fail to indicate direct contact between the activation triplet on KCNE1 and these S6 sites (Kang et al., 2008; Gofman et al., 2012; Xu et al., 2013), suggesting that the functional coupling may be mediated allosterically. Interestingly, KCNE1 also affects S4 movement (Rocheleau and Kobertz, 2008; Wu et al., 2010a; Nakajo and Kubo, 2014) and shifts VSD voltage-dependence of activation to more negative voltages (Osteen et al., 2010; Ruscic et al., 2013; Barro-Soria et al., 2014). A model of KCNE1 regulation has recently been proposed, in which KCNE1 alters VSD-PD coupling interactions to suppress the IO state and modulate the AO state (Zaydman et al., 2014). This model accounts for many of the observed effects of KCNE1 on KCNQ1, including KCNE1-induced inhibition of inactivation. However, this model is not structurally elaborated.

\section{hERG}

Previous studies indicate that KCNE1 (Mcdonald et al., 1997) and KCNE2 (Abbott et al., 1999) can interact with and modulate hERG function. However, whether this interaction occurs under physiological conditions is debated (Weerapura et al., 2002; Anantharam and Abbott, 2005; Abbott et al., 2007). Although KCNE1 and KCNE2 alter hERG gating kinetics in vivo (Mcdonald et al., 1997; Mazhari et al., 2001), co-expression of KCNE2 with hERG in vitro does not reproduce the native $\mathrm{I}_{\mathrm{Kr}}$ current (Weerapura et al., 2002). This suggests that KCNE1 and KCNE2 may not be essential for hERG channel function or that additional factors are required for KCNE-hERG interaction in cardiac cells. The latter possibility is supported by the observation that mutations in KCNE2 may predispose patients to drug-induced LQTS (Abbott et al., 1999; Sesti et al., 2000). As hERG is particularly drug-sensitive, this finding suggests that hERG may, in fact, be modulated by KCNEs in native tissue. Additionally, a T10M mutation in KCNE2 causes arrhythmia induced by auditory stimulation, a known trigger of LQT2, (Gordon et al., 2008). Further studies are needed to resolve these conflicting results and clarify the role of KCNE proteins in regulation of hERG function.

\section{SCN5A}

In humans there are five $\mathrm{Na}_{\mathrm{V}}-\beta$-subunit protein isoforms encoded by four genes, SCN1B-SCN4B ( $\beta 1$ to $\beta 4$ ) (Detta et al., 2015; O'malley and Isom, 2015). All are expressed in the heart and have been shown to associate with SCN5A in heterologous conditions (Makita et al., 1996; Dhar Malhotra et al., 2001; Malhotra et al., 2004; Medeiros-Domingo et al., 2007; Watanabe et al., 2009; Valdivia et al., 2010). $\beta 1$ to $\beta 4$ are single-span transmembrane proteins containing an extracellular N-terminal immunoglobulin (Ig) domain, while $\beta 1 \mathrm{~B}$, a splice variant of SCN1B, lacks the transmembrane domain (Brackenbury and Isom, 2011). Generally, $\beta$-subunits modulate the biophysical properties and cell surface expression 
of $\mathrm{Na}_{\mathrm{V}}$ channels in heterologous cells (Calhoun and Isom, 2014). $\beta 1$ and $\beta 3$ interact non-covalently with $\mathrm{Na}_{\mathrm{V}}$ channels (Meadows et al., 2001), while $\beta 2$ and $\beta 4$ are covalently bound through cysteine bonds between the extracellular Ig domain and channel pore loops. However, modulation of SCN5A by $\beta$-subunits has been difficult to assess. Varying effects of $\beta 1$ (Qu et al., 1995; Dhar Malhotra et al., 2001; Baroni et al., 2014; Zhu et al., 2017) as well as $\beta 3$ (Hu et al., 2009; Valdivia et al., 2010; Wang et al., 2010) on SCN5A have been reported. The rat SCN5A structure provides a potential explanation for this difficulty, as SCN5A is missing a cysteine residue critical for covalent interaction with $\beta 2$, and contains a glycosylation site that may sterically occlude $\beta 1$ interaction (Jiang et al., 2020). SCN5A may not bind tightly with any $\beta$-subunit. Nevertheless, the potential importance of $\beta$ subunits in SCN5A modulation has been suggested by arrhythmia-associated mutations in all four $\beta$-subunit genes, including mutations causing Brugada syndrome (Watanabe et al., 2008; Hu et al., 2009; Hu et al., 2012), LQT3 (MedeirosDomingo et al., 2007; Riuro et al., 2014), and atrial fibrillation (Watanabe et al., 2009; Olesen et al., 2011). However, a recent review of genetic evidence supporting these associations has disputed the clinical validity of $\beta$ subunits as monogenic causes of arrhythmia syndromes (Hosseini et al., 2018; Adler et al., 2020).

SCN5A subcellular localization also contributes importantly to channel regulation. SCN5A channels in cardiac tissue are localized at the lateral membranes and at the anchoring junction between cardiomyocytes (the intercalated disc). These expression patterns give rise to distinct sets of protein-protein interactions and biophysical properties (Lin et al., 2011; Shy et al., 2013). At the lateral membrane SCN5A interacts with the dystrophin/ syntrophin multicomplex, but at the intercalated disc SCN5A interacts with ankyrin-G (Lemaillet et al., 2003; Mohler et al., 2004), which links the channel to cytoskeletal proteins such as actin and the desmosomal protein plakophilin-2 (Makara et al., 2014). Intriguingly, the functional properties of SCN5A channels between these two pools also differ: SCN5A at the lateral membrane has smaller current amplitude, distinct voltagedependence, and slower recovery from inactivation as compared to channels in the intercalated disc (Lin et al., 2011). The functional implications of these differences are not yet clear and remain an active area of study.

\section{Lipid Molecules KCNQ1}

KCNQ1 and other KCNQ channels are dependent on phosphatidyl-4,5-bisphosphate $\left(\mathrm{PIP}_{2}\right)$ for function (Loussouarn et al., 2003; Zaydman et al., 2013; Taylor and Sanders, 2017), and are inhibited upon stimulation of $\mathrm{G}_{\mathrm{q}}$ - and $\mathrm{G}_{11}$-protein coupled receptors, which trigger phospholipase C-catalyzed $\mathrm{PIP}_{2}$ hydrolysis (Selyanko et al., 2000; Loussouarn et al., 2003; Zhang et al., 2003) (Figure 8B). Growing evidence suggests that $\mathrm{PIP}_{2}$ acts as a coupling element for KCNQ1, enhancing weak allosteric interactions between the VSD and PD (Vardanyan and Pongs, 2012; Zaydman et al., 2013; Kasimova et al., 2015; Cui, 2016). Structure-function studies, confirmed by

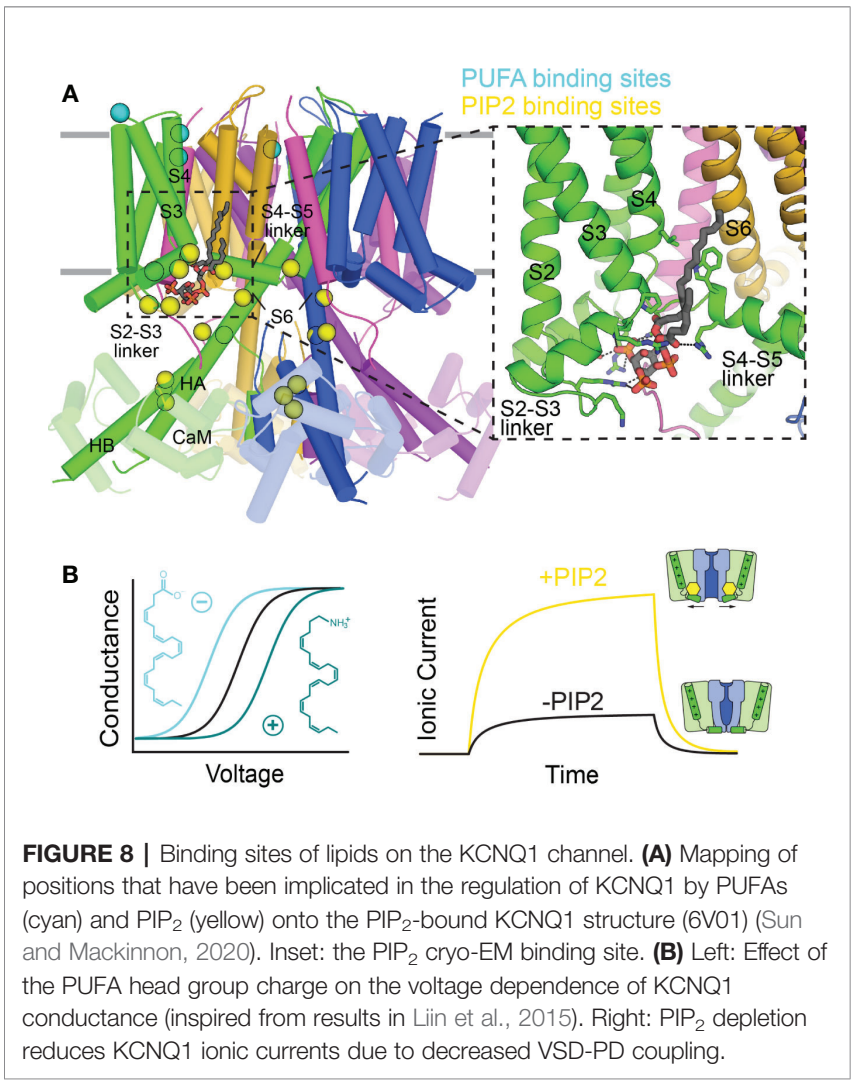

the human KCNQ1/PIP 2 structure, localize $\mathrm{PIP}_{2}$ binding to the cleft between neighboring channel subunits, with interactions involving mostly positively-charged residues in the S2-S3 linker, S4-S5 linker, and S6 (Figure 8A) (Thomas et al., 2011; Zaydman et al., 2013; Eckey et al., 2014; Chen et al., 2015; Sun and Mackinnon, 2020). This binding site seems well-suited to modulate coupling of VSD movement to the activation gate. Questions remain regarding the mechanism of $\mathrm{PIP}_{2}$ regulation of VSD-PD coupling, $\mathrm{PIP}_{2}: \mathrm{KCNQ} 1$ stoichiometry, and binding site differences between the activated and resting states.

$\mathrm{PIP}_{2}$ also binds to the CTD at a site shared with CaM (Tobelaim et al., 2017b). These two regulators may competitively regulate KCNQ1 at this site (Tobelaim et al., 2017a). Indeed, in the human KCNQ1 cryo-EM structure, CaM exhibits a nearly $180^{\circ}$ rotation when $\mathrm{PIP}_{2}$ is bound, losing contact with the S2-S3 linker (Sun and Mackinnon, 2020). Additionally, S6 and HA in KCNQ1 form a single helix in the open channel. These structural shifts point to an interplay between $\mathrm{PIP}_{2}$ and $\mathrm{CaM}$, but does not clarify the nature of this interaction, as no $\mathrm{PIP}_{2}$ density was seen at the CTD binding site in the cryo-EM structure. The details of the coordination between CaM and $\mathrm{PIP}_{2}$, and its role in channel function, remain to be elucidated.

Polyunsaturated fatty acids (PUFAs) also modulate KCNQ1 function (Taylor and Sanders, 2017). While PUFAs generally inhibit ion channel current (Boland and Drzewiecki, 2008), the KCNQ1-KCNE1 channel is a notable exception. The $\mathrm{I}_{\mathrm{Ks}}$ current is enhanced by docosahexaenoic acid (DHA) and, to a lesser 
extent, oleic acid (Doolan et al., 2002) by shifting the conductance-vs-voltage curve $(\mathrm{GV})$ to more negative voltages. Interestingly, the charge of the head group determines the direction of the (GV) shift (Figure 8B): negatively-charged DHA causes a negative shift, a neutral head group has no effect, and a positively-charged one shifts the (GV) curve to positive potentials, reducing channel function (Liin et al., 2015). Negative head group charge and a polyunsaturated acyl chain appear to be required for channel activation. The PUFA binding site in KCNQ1 appears to involve residues in the extracellular S3-S4 loop, R1 and R2 on S4, and the PD (Figure 8A), as mutations in these regions either reduce the PUFA effect or completely abolish the (GV) shift (Liin et al., 2015; Liin et al., 2018).

\section{hERG}

As with KCNQ1, the function of hERG is upregulated by $\mathrm{PIP}_{2}$ by means of increased current amplitude, a hyperpolarizing shift in voltage-dependence of activation, as well as faster activation and slower inactivation rates (Bian et al., 2001). Stimulation of $\mathrm{G}_{\mathrm{\alpha q}^{-}}$ protein coupled receptors also suppresses the $\mathrm{I}_{\mathrm{Kr}}$ current through $\mathrm{PIP}_{2}$ depletion (Bian et al., 2001; Bian et al., 2004). Binding of $\mathrm{PIP}_{2}$ to hERG likely localizes to a cluster of basic residues (R883Q900) C-terminal to the CNBHD, as substitution of these residues to neutral or negatively-charged amino acids prevented $\mathrm{PIP}_{2}$ effects on hERG function and abolished $\mathrm{PIP}_{2}$ binding (Bian et al., 2004). Unfortunately, these residues are not resolved in the available hERG structure (Wang and Mackinnon, 2017).

\section{SCN5A}

In contrast to KCNQ1, PUFAs such as eicosapentaenoic acid (EPA), docosahexaenoic acid (DHA), linoleic acid (LA), and $\alpha$ linolenic acid (ALA) suppress $\mathrm{I}_{\mathrm{Na}}$ current in a concentrationdependent manner by shifting the voltage dependence of $\mathrm{I}_{\mathrm{Na}}$ inactivation to more hyperpolarized potentials (Kang et al., 1995; Kang et al., 1997; Leifert et al., 1999). EPA also accelerates the transition from the resting state to the inactivated state and slows recovery from inactivation (Xiao et al., 1998; Isbilen et al., 2006). However, the effect was reduced by $\beta 1$ subunit expression (Xiao et al, 2000). Furthermore, N406K renders SCN5A less sensitive to inhibition by EPA, an effect strengthened by $\beta 1$ subunit expression (Xiao et al., 2001). The structural basis for PUFA binding and modulation is not well understood.

\section{CHANNEL DYSFUNCTION IN CONGENITAL LONG QT SYNDROME}

Alterations in the action potential disturb impulse propagation and cause reentry (Kleber Ag, 2004), whereby the impulse restimulates the heart tissue that generated it. Reentry promotes cardiac arrhythmias and predisposes to sudden unexplained death (Skinner et al., 2019). LQTS is a prevalent cause of such events, with an estimated population prevalence of approximately 1:2500 (Schwartz et al., 2009).
LQTS is characterized by a prolonged rate-corrected QT interval in patient electrocardiograms (ECGs), indicative of impaired repolarization (Schwartz et al., 2012). In a large proportion of cases, QT interval prolongation is the result of either a loss-of-function in KCNQ1 or hERG, or a gain-offunction in the SCN5A channel (Moss and Kass, 2005; Schwartz et al., 2012; Earle et al., 2013; Skinner et al., 2019). Mutations in these genes confer distinct subtypes of LQTS (denoted LQT1-3, respectively), each with unique ECG features, risk factors, arrhythmia triggers, and responsiveness to $\beta$-adrenergic receptor blockers, the most common LQTS treatment (Ackerman, 2005; Moss and Kass, 2005; Skinner et al., 2019; Wallace et al., 2019). Below, the function of each ion channel in the cardiac action potential is discussed, along with how each can cause action potential dysfunction in LQTS.

\section{Channel Function in the Cardiac Action Potential and Dysfunction in LQTS KCNQ1}

In complex with KCNE1, KCNQ1 conducts $\mathrm{I}_{\mathrm{Ks}}$, which helps shape the plateau and repolarization phases of the action potential, in part by counteracting calcium influx (Skinner et al., 2019). Due to slow activation, the KCNQ1-KCNE1 channel is only slightly open during the plateau phase, with $\mathrm{I}_{\mathrm{Ks}}$ increasing slowly in a nearly linear fashion (Figure 7C) until the repolarization phase is reached and the channel becomes fully activated (Figures 1B, C) (Moss and Kass, 2005). KCNQ1KCNE1 channels do not inactivate (Pusch et al., 1998), conducting current throughout activation and deactivation. The channel slowly deactivates, shaping the tail of the repolarization phase and mediating return to the resting potential (Moss and Kass, 2005; George, 2013; Skinner et al., 2019). Thus, loss-of-function in KCNQ1 prolongs repolarization primarily by preventing completion of the repolarization phase, broadening the tail of the T-wave (Figure 1A) and causing type 1 LQTS (LQT1). (Ackerman, 2005; Tester and Ackerman, 2014; Skinner et al., 2019; Wallace et al., 2019). The effects of a KCNQ1 mutation are exacerbated upon sympathetic activation of $\beta$ adrenergic receptors due to $\mathrm{I}_{\mathrm{Ks}}$ potentiation, while resting heart rates show few perturbations (Shimizu and Antzelevitch, 1998). The QT interval is unable to shorten in response to this stimulation, making physical exertion accompanied by heightened sympathetic nervous system activity a prevalent LQT1 trigger (Schwartz et al., 2012; Bohnen et al., 2017).

\section{hERG}

hERG makes up the other primary inward rectifier current $I_{\mathrm{Kr}}$, which acts in both the plateau and the repolarization phase (Figures 1B, C) (Moss and Kass, 2005; Skinner et al., 2019). As the channel activates, rapid entry into and out of inactivation (due to SF fluctuations) creates a persistent current through the plateau phase until slow deactivation, regulated by the PAS and CNBHD domains, closes the channel during the repolarization phase. While both hERG and KCNQ1 are conductive during the plateau and repolarization phases, hERG has a higher unitary conductance than KCNQ1 in both phases (Moss and Kass, 2005; 
George, 2013). The resulting $\mathrm{I}_{\mathrm{Kr}}$ current thus supplies a greater proportion of the overall $\mathrm{I}_{\mathrm{K}}$ current (Cheng and Kodama, 2004), with loss-of-function in hERG (LQT2) correlating with a lower $\mathrm{T}$-wave amplitude in contrast to the normal T-wave amplitude associated with LQT1. Additionally, LQT2 is often triggered at rest (Skinner et al., 2019), consistent with the greater hERG contribution to $\mathrm{I}_{\mathrm{K}}$. However, while $\mathrm{I}_{\mathrm{Kr}}$ is usually more prominent (Cheng and Kodama, 2004), the relative density of $\mathrm{I}_{\mathrm{Kr}}$ and $\mathrm{I}_{\mathrm{Ks}}$ vary by ventricular cell type (Liu and Antzelevitch, 1995; Viswanathan et al., 1999).

\section{SCN5A}

SCN5A produces the $\mathrm{I}_{\mathrm{Na}}$ current that shapes the initial upstroke of the cardiac action potential (Skinner et al., 2019), initiating depolarization. SCN5A is rapidly activated through asynchronous motion of the VSD's in repeats I-III coupled to pore opening, then quickly inactivates through interaction of the IFM motif with the channel, resulting in a brief inward spike of sodium current that depolarizes the cell (Ghovanloo et al., 2016) (Figures 1B, C). Unlike KCNQ1 and hERG, LQT3 is conferred by SCN5A gain-of-function mutations that cause a "leaky" inward sodium current (Wilde and Amin, 2018; Skinner et al., 2019). The functional $I_{K r}$ and $I_{K s}$ outward currents are unable to compensate for the persistent inward $\mathrm{I}_{\mathrm{Na}}$ current, prolonging entry into and completion of the repolarization phase and giving rise to LQT3. As in LQT2, LQT3-related arrhythmias occur most often at rest, rather than in response to stress or exercise (Schwartz et al., 2012). It should be noted that while loss-offunction mutations for SCN5A are not relevant to LQTS, they are prevalent in other channelopathies, particularly Brugada syndrome (Wilde and Amin, 2018; Skinner et al., 2019).

\section{Molecular Mechanisms of LQTS}

Mutations in KCNQ1, hERG, and SCN5A can lead to LQTS by perturbing channel function through distinct molecular mechanisms. Structural studies of these channels can help tease apart these molecular mechanisms for a more detailed understanding of LQTS. We have mapped LQTS mutations identified as pathogenic according to ClinVar (Harrison et al., 2016) and HGMD (Stenson et al., 2012) databases onto the human KCNQ1 (Sun and Mackinnon, 2020) and hERG structures (Wang and Mackinnon, 2017), as well as a recent model of SCN5A (Kroncke et al., 2019) and the rat SCN5A homolog (Jiang et al., 2020) (Figures 9-11). The list of curated mutations is found in Table 1: 261 mutations in KCNQ1, 320 mutations in hERG, and 122 mutations in SCN5A. Examination of these mutation sites provides some insight into the channel mechanisms that may be commonly disrupted in LQT1-3.

\section{KCNQ1}

KCNQ1 mutations studied to date are highly varied in molecular effects, with the potential to induce defects in channel stability, trafficking, electrophysiology, or all three (Chen et al., 2011; Heijman et al., 2012; Wu et al., 2016; Bohnen et al., 2017). So far, a strong disposition for either expression or functional defects has not been elucidated. However, analysis of mutation sites points to regions of increased pathological risk and provides hints regarding prevalent mechanisms.

LQT1-associated mutations are found in every domain of the protein (Figure 9A), particularly in the transmembrane channel domain (Shimizu et al., 2004; Moss et al., 2007; Kapa et al., 2009). Mutations in the SF, (Figure 9A, inset), VSD (Figure 9B), and the S4-S5 linker (Figure 9C) are especially prominent.

Mutations in and around the SF (Figure 9A, inset) point to a potential ion permeation defect, as conformational changes may prevent potassium ion permeation and thus decrease the effective $\mathrm{I}_{\mathrm{Ks}}$ current (Choveau and Shapiro, 2012), as noted in several SF mutations (Ikrar et al., 2008; Thomas et al., 2010; Burgess et al., 2012; Chen et al., 2019). Molecular dynamics simulations of analogous mutations in KcsA indicated that mutations in the SFinteracting residues (including T322M, T322A, or G325R) may disrupt the organization of backbone atoms in the SF, abolishing $\mathrm{K}^{+}$ion coordination and producing a dominant-negative effect on channel current (Burgess et al., 2012).

VSD mutations are more common in KCNQ1 compared to hERG and SCN5A (see Figures 9B, 10B, and 11A), indicating that the VSD is more important in LQT1. Analysis of LQTS mutations located in the VSD (Huang et al., 2018; Vanoye et al., 2018) indicate that loss of channel function was most commonly a consequence of mutation-induced destabilization of the channel, resulting in mistrafficking and lower surface expression. These results strongly correlated with Rosetta energy calculations of VSD mutants (Kuenze et al., 2019), supporting the importance of VSD destabilization as a LQT1 mechanism. A number of the deleterious mutations were found in the S0 helix and S0-contacting regions of the VSD. Among the 50 VSD mutations studied, the S0 mutations had the largest energetic destabilizations consistent with the large number of contacts formed between S0 and other regions of the VSD. (Huang et al., 2018; Kuenze et al., 2019). However, these studies also identified variants that did not exhibit folding and trafficking defects, but were either non-functional (low conductance) or dysfunctional, with modified gating, again pointing to a complex spectrum of LQT1 disease mechanisms.

Mutations in the KCNQ1 S4-S5 linker and its contacting regions (Figure 9C) have the potential to perturb VSD-PD coupling and channel gating. Such effects have been demonstrated for a number of LQTS-associated mutations in the S4-S5 linker and the cytoplasmic end of S6, particularly for $\mathrm{R} 243 \mathrm{C}, \mathrm{W} 248 \mathrm{~F} / \mathrm{R}$, and Q357R, producing positive shifts in the voltage dependence of activation or inactivation and and/or decreased current amplitude (Franqueza et al., 1999; Boulet et al., 2006; Mousavi Nik et al., 2015). Because the S4-S5 linker is involved in both VSD/PD interactions and in binding of $\mathrm{PIP}_{2}$ (Eckey et al., 2014; Sun and Mackinnon, 2017) and KCNE1 (Kang et al., 2008; Xu et al., 2013; Cui, 2016), mutations in this region may affect gating either by weakening VSD-PD coupling or by hindering modulation by auxiliary molecules. Some LQT1 variant-induced changes in $\mathrm{PIP}_{2}$ binding at the S4-S5 linker have been noted (including R243H, R539W, and R555C) (Park et al., 2005). Due to the complex nature of electromechanical coupling 


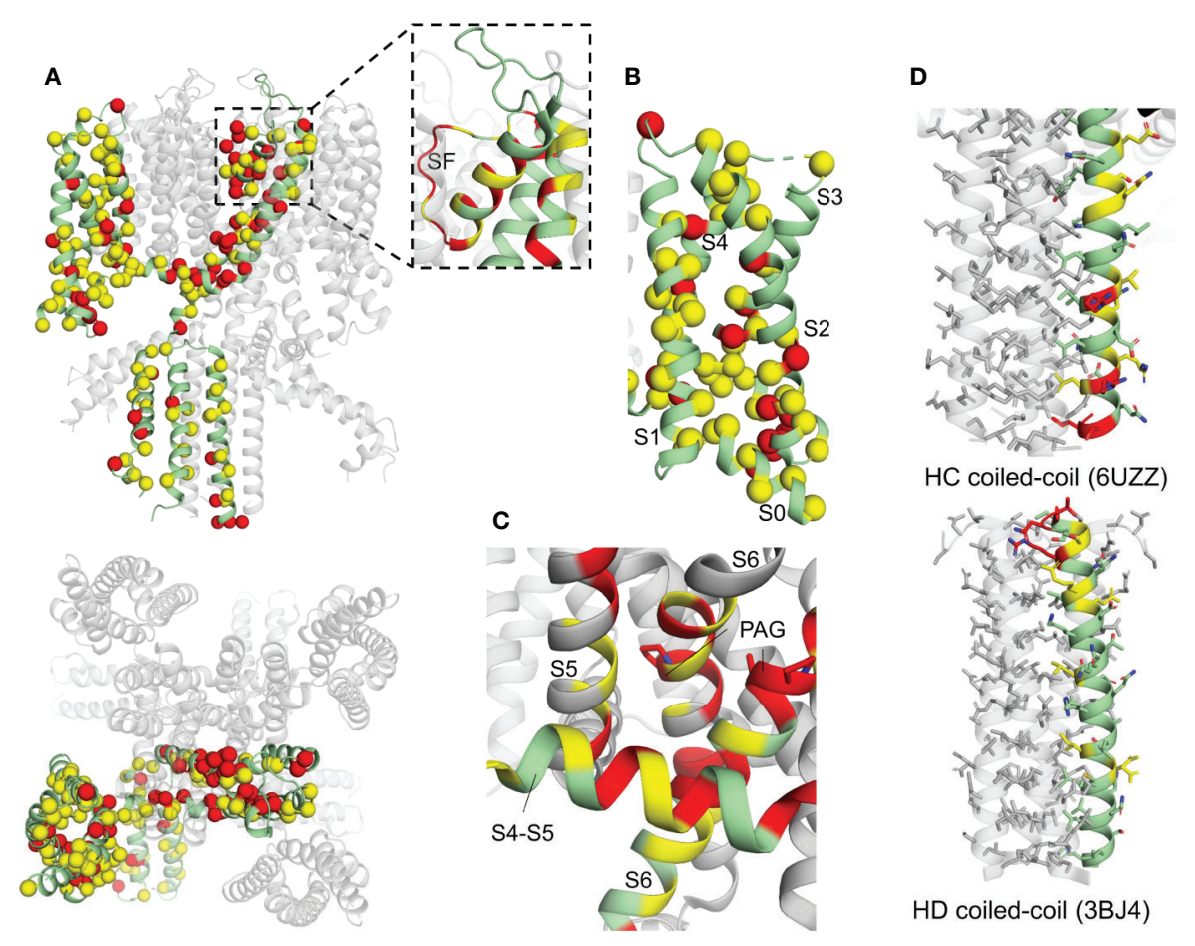

FIGURE 9 | Human KCNQ1 structure with deleterious mutation sites indicated. (A) Full KCNQ1 structure (PDB: 6UZZ) (Sun and Mackinnon, 2020). Yellow sites denote locations of a single deleterious mutation, while red indicates sites with multiple mutations identified. Spheres indicate C-alpha positions of mutation sites. Subunit with LQT1 mutations sites mapped is colored green. Inset: of SF and pore helix. (B) KCNQ1 VSD. (C) S4-S5 linker (S4-S5) interaction site close-up. One subunit is colored green and the others in gray, for clarity. The PAG motif (PAG) is indicated with sticks. (D) Top: HC coiled-coil domain. Bottom: HD coiled-coil domain (PDB: 3BJ4) (Wiener et al., 2008). Residue side chains are indicated with sticks.

in KCNQ1 and the requirement for auxiliary subunits, a detailed structural understanding of mutation effects in this region is particularly challenging. However, the human KCNQ1, KCNQ1KCNE3, and KCNQ1-KCNE3/PIP 2 channel structures (Sun and Mackinnon, 2020) may provide guidance in experimental design to elucidate LQT1-associated coupling defects in this region.

While the majority of LQT1 mutations map to the transmembrane domain, mutations are also found in the CTD, both in the HA/HB helix bundle, where CaM binds, and in the $\mathrm{HC}$ and HD coiled-coil domains (Figure 9A). Mutations in HC and HD are particularly prevalent in sites of hydrophobic and electrostatic interactions that stabilize the coil, hinting at channel assembly defects (Figure 9D). Defects in CaM binding and tetramerization cause some forms of LQT1, as demonstrated for several mutations (Kanki et al., 2004; Ghosh S, 2006; Schwake et al., 2006; Wiener et al., 2008).

\section{hERG}

Loss-of-function mutations in hERG may lead to folding/ trafficking defects, gating defects, or both (Anderson et al., 2014; Smith et al., 2016; Bohnen et al., 2017). However, as many as $90 \%$ of hERG loss-of-function mutations appear to cause trafficking defects, independent of which domain is mutated (Smith et al., 2016). As in KCNQ1, pore domain mutations in hERG demonstrated stronger dominant-negative effects in hERG than other regions, hinting that mutations in this region are more severe (Anderson et al., 2014).

Although hERG mutations span the entire protein, they are prominent in the SF (Figure 10A, inset), the extracellular VSD/ $\mathrm{PD}$ interface between S1 and S5 (Figure 10C), and in the PAS and CNBHD cytoplasmic domains (Figure 10D). Given the inherent instability of the hERG SF (Fan et al., 1999; Stansfeld et al., 2008; Vandenberg et al., 2012) and the functional necessity of the SF for fast inactivation (Herzberg et al., 1998; Hoshi and Armstrong, 2013), mutations may act by both affecting channel stability and inactivation. Inactivation defects due to LQT2associated pore helix mutations have been identified (Zhao et al., 2009; Poulsen et al., 2015). However, mutations in LQT2 more commonly cause misfolding and/or trafficking defects. Potassium ions appear to stabilize the mature hERG channel (possibly by interacting with the SF) to promote trafficking (Wang et al., 2009; Apaja et al., 2013), with intracellular $\mathrm{K}^{+}$ depletion resulting in ER retention. G601S mimicked this phenotype (Apaja et al., 2013). G601S, a mutation in the pore loop near the SF, mimicked also impedes trafficking through enhanced chaperone association in the ER (Ficker et al., 2003), supporting the idea of SF destabilization as a cause for channel mistrafficking. A detailed understanding of mutations in the SF region requires both intensive structural and cellular analysis to uncover not only protein conformational changes upon 


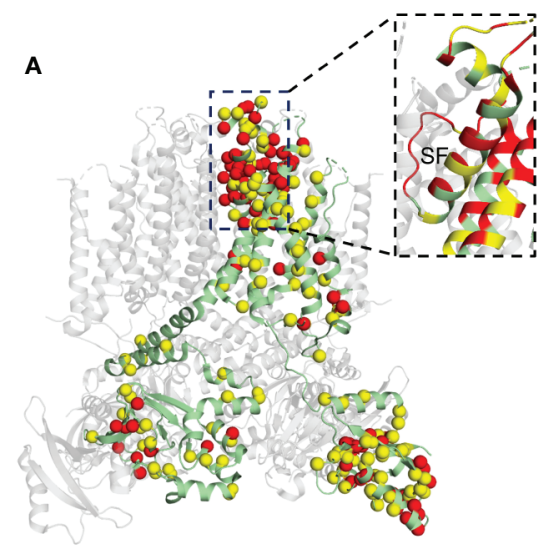

B $\quad$ C
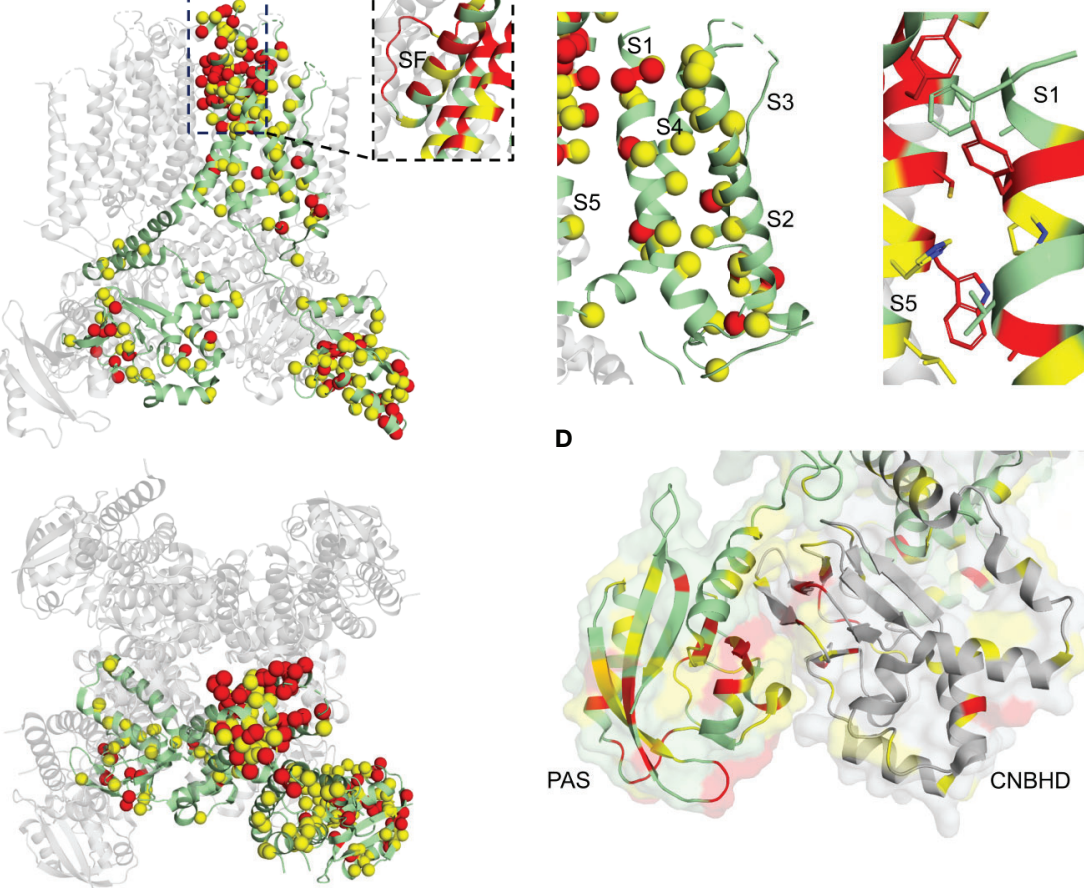

FIGURE 10 | hERG structure with deleterious mutation sites indicated. (A) Full hERG structure (PDB: 5VA1) (Wang and Mackinnon, 2017). Yellow sites denote locations of a single deleterious mutation, while red indicates sites with multiple mutations identified. Spheres indicate C-alpha positions of mutation sites. Subunit with LQT2 mutations sites mapped is colored green. Inset: close-up of SF and pore helix. (B) hERG VSD. (C). Close-up of the S1/S5 interface. Residues at the interface are indicated with sticks (D) N-terminal PAS (dark gray) and C-terminal CNBHD (light gray) domain interface, cartoon representation with surface representation overlay. One subunit is green and the other gray, for clarity.

A

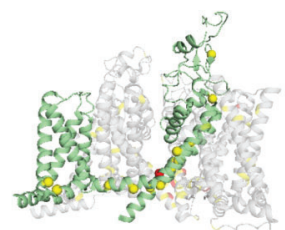

Repeat I

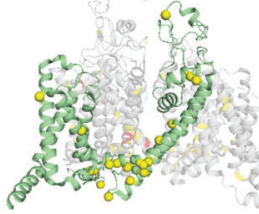

Repeat III

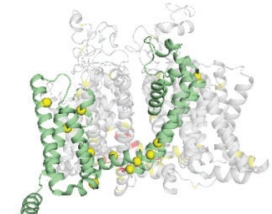

ह

Repeat II

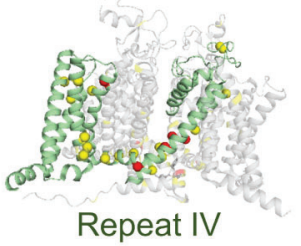

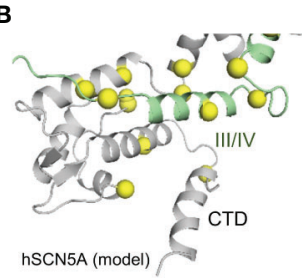

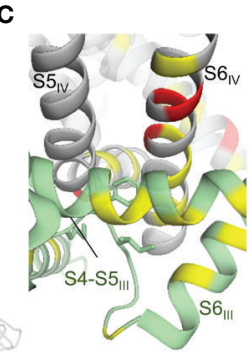

D

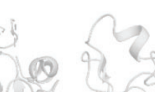

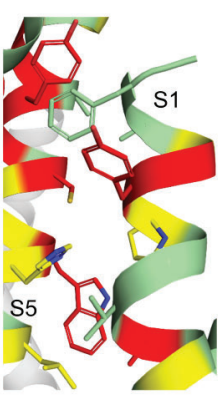


TABLE 1 | LQTS mutations in KCNQ1, hERG, and SCN5A.

\begin{tabular}{|c|c|c|c|c|c|c|c|c|c|c|c|}
\hline \multicolumn{4}{|c|}{ KCNQ1 } & \multicolumn{4}{|c|}{ hERG } & \multicolumn{4}{|c|}{ SCN5A } \\
\hline Mutant & Database & Mutant & Database & Mutant & Database & Mutant & Database & Mutant & Database & Mutant & Database \\
\hline $\mathrm{A}^{4} 8 \mathrm{P}^{\star}$ & HGMD & T311I & HGMD & G6R & HGMD & G572R & ClinVar, HGMD & $\mathrm{G} \mathrm{V}^{*}$ & HGMD & G1329S & HGMD \\
\hline $\mathrm{S} 6 \mathrm{~F}^{\star}$ & HGMD & T312S & HGMD & G6V & HGMD & G572S & HGMD & $\mathrm{R} 18 \mathrm{~W}^{*}$ & HGMD & A1330P & HGMD \\
\hline T96R* & HGMD & T312I & ClinVar, HGMD & $\mathrm{T} 13 \mathrm{~N}$ & HGMD & G572V & HGMD & A29V* & HGMD & A1330T & HGMD \\
\hline T104I & HGMD & I313M & HGMD & D16A & HGMD & M574V & HGMD & $\mathrm{E} 30 \mathrm{G}^{*}$ & HGMD & P1332L & HGMD \\
\hline Q107H & HGMD & G314A & HGMD & R20G & HGMD & E575G & HGMD & P52S* & HGMD & S1333Y & HGMD \\
\hline R109L & HGMD & G314D & HGMD & F22S & HGMD & E575K & HGMD & R53Q* & HGMD & L1338V & HGMD \\
\hline Y111C & ClinVar, HGMD & G314C & HGMD & $\mathrm{F} 22 \mathrm{Y}$ & HGMD & $\mathrm{R} 582 \mathrm{C}^{\star}$ & ClinVar & $\mathrm{R}_{104 \mathrm{G}^{*}}$ & HGMD & A1357V & HGMD \\
\hline L114P & HGMD & G314R & ClinVar, HGMD & S26l & HGMD & G584S & HGMD & $\mathrm{A} 110 \mathrm{~T}^{*}$ & HGMD & G1391R & HGMD \\
\hline E115G & HGMD & G314S & ClinVar, HGMD & $\mathrm{R} 27 \mathrm{P}$ & HGMD & G584V & HGMD & V1131* & HGMD & A1428S & HGMD \\
\hline P117L & ClinVar, HGMD & Y315N & HGMD & K28E & HGMD & W585C & HGMD & $\mathrm{S} 115 \mathrm{G}^{\star}$ & HGMD & S1458Y & ClinVar, HGMD \\
\hline C122Y & ClinVar, HGMD & Y315C & HGMD & F29L & HGMD & L586M & HGMD & I176M & HGMD & N1472S & HGMD \\
\hline Y125D & HGMD & Y315H & HGMD & F29S & HGMD & N588D & HGMD & A185T & HGMD & F1473C & HGMD \\
\hline F127L & HGMD & Y315F & HGMD & I31S & HGMD & G590V & HGMD & I239V & HGMD & F1473S & HGMD \\
\hline L131P & HGMD & Y315S & HGMD & I31T & ClinVar, HGMD & I593T & ClinVar & V240M & HGMD & Q1476R & HGMD \\
\hline I132L & HGMD & G316E & HGMD & А32T & HGMD & I593K & ClinVar, HGMD & Q245K & HGMD & G1481E & HGMD \\
\hline V133I & HGMD & G316V & HGMD & N33T & HGMD & I593R & ClinVar, HGMD & V258A & HGMD & T1488R & HGMD \\
\hline L134P & ClinVar, HGMD & G316R & ClinVar, HGMD & R35W & HGMD & I593V & HGMD & R340Q & HGMD & Y1495S & HGMD \\
\hline C136F & HGMD & D317N & ClinVar, HGMD & V41F & HGMD & G594D & HGMD & A385T & HGMD & $\mathrm{K} 1505 \mathrm{~N}$ & HGMD \\
\hline L137F & ClinVar, HGMD & D317G & HGMD & V41A & HGMD & K595N & HGMD & I397T & HGMD & T1544P & HGMD \\
\hline S140R & HGMD & D317Y & HGMD & $142 \mathrm{~N}$ & HGMD & K595E & HGMD & L404Q & HGMD & L1560F & HGMD \\
\hline E146G & HGMD & K318N & ClinVar, HGMD & Y43C & HGMD & $\mathrm{P} 596 \mathrm{H}$ & ClinVar, HGMD & N406K & HGMD & I1593M & HGMD \\
\hline E146K & HGMD & P320A & HGMD & Y43D & HGMD & P596A & ClinVar, HGMD & L409P & HGMD & F1594S & HGMD \\
\hline A150G & HGMD & T322K & HGMD & $\mathrm{C} 44 \mathrm{~F}$ & HGMD & P596R & HGMD & L409V & HGMD & V1597M & HGMD \\
\hline T153M & HGMD & T322A & ClinVar, HGMD & C44W & HGMD & P596L & HGMD & V411M & ClinVar, HGMD & S1609W & HGMD \\
\hline F157C & HGMD & T322M & ClinVar, HGMD & C44Y & HGMD & P596S & HGMD & A413E & HGMD & $\mathrm{R} 1623 \mathrm{Q}$ & ClinVar, HGMD \\
\hline E160K & HGMD & G325R & ClinVar, HGMD & N45D & HGMD & P596T & HGMD & $\mathrm{R} 504 \mathrm{~T}^{\star}$ & HGMD & R1623L & HGMD \\
\hline E160V & HGMD & G325E & HGMD & N45S & HGMD & Y597C & HGMD & M506K & HGMD & $\mathrm{R} 1626 \mathrm{P}$ & HGMD \\
\hline G168R & ClinVar, HGMD & G325W & HGMD & N45Y & HGMD & G604D & HGMD & F530V* & HGMD & $\mathrm{R} 1644 \mathrm{H}$ & ClinVar, HGMD \\
\hline T169R & HGMD & S338F & HGMD & D46Y & HGMD & G604S & ClinVar, HGMD & D536 $\mathrm{H}^{\star}$ & HGMD & L1646R & HGMD \\
\hline T169K & HGMD & F339S & HGMD & G47D & HGMD & P605S & HGMD & R569W* & HGMD & L1650F & HGMD \\
\hline E170G & HGMD & F339V & HGMD & G47V & HGMD & S606F & HGMD & Q573E ${ }^{\star}$ & HGMD & M1652R & HGMD \\
\hline V173D & HGMD & F340L & HGMD & C49Y & HGMD & S606P & HGMD & G579R* $^{*}$ & HGMD & M1652T & HGMD \\
\hline $\mathrm{R} 174 \mathrm{C}$ & ClinVar, HGMD & A341G & HGMD & G53R & HGMD & D609G & HGMD & P637L* & HGMD & P1725L & HGMD \\
\hline $\mathrm{R} 174 \mathrm{H}$ & ClinVar, HGMD & A341V & ClinVar, HGMD & G53D & HGMD & $\mathrm{D} 609 \mathrm{H}$ & HGMD & E654K* & HGMD & $\mathrm{A} 1746 \mathrm{~T}$ & HGMD \\
\hline W176R & HGMD & A341E & ClinVar, HGMD & G53S & HGMD & D609N & ClinVar, HGMD & A665S* & HGMD & I1758V & HGMD \\
\hline A178T & HGMD & L342F & HGMD & G53V & HGMD & D609Y & HGMD & $\mathrm{R} 69 \mathrm{C}^{\star}$ & HGMD & $\mathrm{L} 1761 \mathrm{H}$ & HGMD \\
\hline A178P & ClinVar, HGMD & P343R & HGMD & $\mathrm{Y} 54 \mathrm{H}$ & HGMD & K610N & HGMD & G709V* & HGMD & L1761F & HGMD \\
\hline K183R & HGMD & P343L & HGMD & S55L & ClinVar, HGMD & Y611D & HGMD & T731I & HGMD & V1763L & HGMD \\
\hline K183M & HGMD & P343S & HGMD & $\mathrm{R} 56 \mathrm{Q}$ & HGMD & Y611H & ClinVar, HGMD & Q750R & HGMD & V1763M & ClinVar, HGMD \\
\hline Y184C & HGMD & A344E & HGMD & A57P & HGMD & V612L & HGMD & Q779K & HGMD & M1766L & ClinVar, HGMD \\
\hline Y184H & HGMD & A344V & ClinVar, HGMD & E58A & HGMD & V612M & HGMD & $\mathrm{R} 800 \mathrm{~L}^{*}$ & HGMD & M1766V & HGMD \\
\hline Y184S & HGMD & G345R & ClinVar, HGMD & E58D & HGMD & T613A & HGMD & R808P & HGMD & Y1767C & HGMD \\
\hline G186R & HGMD & G345E & ClinVar, HGMD & E58G & ClinVar, HGMD & T613K & HGMD & F816Y & HGMD & I1768V & ClinVar, HGMD \\
\hline G189A & HGMD & G345A & HGMD & E58K & HGMD & T613M & ClinVar, HGMD & L828V & HGMD & L1772V & HGMD \\
\hline G189E & HGMD & G345V & HGMD & Q61R & HGMD & A614V & ClinVar, HGMD & N834D & HGMD & N1774D & HGMD \\
\hline G189R & ClinVar, HGMD & L347P & HGMD & $\mathrm{R} 62 \mathrm{Q}$ & HGMD & L615F & HGMD & G840R & HGMD & E1781G & HGMD \\
\hline $\mathrm{R} 190 \mathrm{Q}$ & ClinVar, HGMD & S349W & ClinVar, HGMD & $\mathrm{C} 64 \mathrm{~W}$ & HGMD & Y616C & HGMD & T843A & ClinVar, HGMD & E1784K & HGMD \\
\hline R190L & HGMD & S349P & HGMD & C64Y & HGMD & F617L & HGMD & Q912R & HGMD & D1790G & HGMD \\
\hline R190W & HGMD & G350R & HGMD & T65P & ClinVar, HGMD & F617V & HGMD & S941N & ClinVar & Y1795C & ClinVar, HGMD \\
\hline L191P & HGMD & G350V & HGMD & C66G & HGMD & T618S & HGMD & Q960K* & HGMD & P1824A & HGMD \\
\hline R192P & HGMD & F351L & ClinVar & F68L & HGMD & S620N & HGMD & $\mathrm{R} 975 \mathrm{~W}^{*}$ & HGMD & D1839G & HGMD \\
\hline F193L & HGMD & F351S & HGMD & L69P & HGMD & S620G & HGMD & $\mathrm{C} 981 F^{\star}$ & HGMD & R1860S & HGMD \\
\hline R195P & HGMD & L353P & ClinVar, HGMD & $\mathrm{H} 70 \mathrm{R}$ & HGMD & S621R & HGMD & P1021S* & HGMD & A1870T & HGMD \\
\hline K196T & HGMD & K354R & HGMD & $\mathrm{H} 70 \mathrm{~N}$ & HGMD & S621N & HGMD & D1166N* & HGMD & R1897W* & HGMD \\
\hline P197S & HGMD & Q357R & HGMD & G71E & HGMD & T623I & HGMD & $\mathrm{R} 1175 \mathrm{C}^{*}$ & HGMD & E1901Q* & HGMD \\
\hline I198V & ClinVar, HGMD & R360M & HGMD & G71R & ClinVar, HGMD & V625A & HGMD & P1177L* & HGMD & A1949S* & HGMD \\
\hline S199A & HGMD & R360T & HGMD & G71W & HGMD & V625E & HGMD & Y1199S* & HGMD & E1954K* & HGMD \\
\hline I200N & HGMD & K362R & HGMD & P72L & ClinVar, HGMD & G626A & HGMD & Y1241S & HGMD & Y1977N* & HGMD \\
\hline D202G & ClinVar & H363N & ClinVar, HGMD & $\mathrm{P} 72 \mathrm{~T}$ & HGMD & G626D & HGMD & I1278N & HGMD & $\mathrm{L}_{1988 R^{*}}$ & HGMD \\
\hline D202H & HGMD & $\mathrm{N} 365 \mathrm{H}$ & HGMD & $\mathrm{T} 74 \mathrm{P}$ & HGMD & G626S & HGMD & N1325S & ClinVar, HGMD & $R 1991 Q^{\star}$ & HGMD \\
\hline L203P & HGMD & R366Q & ClinVar & $\mathrm{T} 74 \mathrm{R}$ & ClinVar, HGMD & G626V & HGMD & A1326S & ClinVar, HGMD & F2004V* & HGMD \\
\hline
\end{tabular}


TABLE 1 | Continued

\begin{tabular}{|c|c|c|c|c|c|c|c|c|c|c|c|}
\hline \multicolumn{4}{|c|}{ KCNQ1 } & \multicolumn{4}{|c|}{ hERG } & \multicolumn{4}{|c|}{ SCN5A } \\
\hline Mutant & Database & Mutant & Database & Mutant & Database & Mutant & Database & Mutant & Database & Mutant & Database \\
\hline I204M & HGMD & R366P & HGMD & A78V & HGMD & F627I & HGMD & & & & \\
\hline I204F & HGMD & R366W & HGMD & A78T & HGMD & F627L & HGMD & & & & \\
\hline V205M & ClinVar, HGMD & Q367H & HGMD & A80P & HGMD & G628R & HGMD & & & & \\
\hline S209F & HGMD & А371T & HGMD & A85P & HGMD & G628D & HGMD & & & & \\
\hline K218E & HGMD & A372D & HGMD & A85V & HGMD & G628S & ClinVar, HGMD & & & & \\
\hline T224M & HGMD & S373P & HGMD & L86R & HGMD & G628V & HGMD & & & & \\
\hline S225L & HGMD & W379G & HGMD & L86P & HGMD & G628A & HGMD & & & & \\
\hline A226V & HGMD & W379S & HGMD & A89V & HGMD & N629D & ClinVar, HGMD & & & & \\
\hline I227L & HGMD & R380G & HGMD & E90K & HGMD & N629| & HGMD & & & & \\
\hline G229D & HGMD & R380S & HGMD & V94M & HGMD & N629K & HGMD & & & & \\
\hline R231C & HGMD & E385K & HGMD & E95G & HGMD & N629S & ClinVar, HGMD & & & & \\
\hline $\mathrm{R} 231 \mathrm{H}$ & ClinVar, HGMD & S389P & HGMD & I96T & HGMD & N629T & HGMD & & & & \\
\hline I235N & ClinVar, HGMD & S389Y & HGMD & F98S & HGMD & V630A & HGMD & & & & \\
\hline L236R & HGMD & T391I & HGMD & Y99S & HGMD & V630L & HGMD & & & & \\
\hline L236P & HGMD & W392R & HGMD & $\mathrm{R} 100 \mathrm{Q}$ & ClinVar, HGMD & S631A & HGMD & & & & \\
\hline L239P & HGMD & Y395S & ClinVar & R100W & HGMD & P632A & HGMD & & & & \\
\hline V241G & HGMD & $\mathrm{K} 422 \mathrm{~T}^{*}$ & HGMD & K101E & HGMD & P632S & HGMD & & & & \\
\hline D242N & HGMD & $\mathrm{T}_{444 \mathrm{M}^{*}}$ & HGMD & D102A & HGMD & N633D & HGMD & & & & \\
\hline D242Y & HGMD & D446E* & HGMD & $\mathrm{D} 102 \mathrm{H}$ & HGMD & N633I & HGMD & & & & \\
\hline $\mathrm{R} 243 \mathrm{C}$ & ClinVar, HGMD & $\mathrm{H} 455 \mathrm{Y}^{*}$ & HGMD & D102V & HGMD & N633K & HGMD & & & & \\
\hline R243P & ClinVar, HGMD & R511W & HGMD & F106L & HGMD & N633S & HGMD & & & & \\
\hline G245V & HGMD & T513S & HGMD & F106Y & HGMD & T634A & HGMD & & & & \\
\hline W248C & HGMD & I517T & HGMD & C108R & HGMD & T634I & HGMD & & & & \\
\hline $\mathrm{L} 250 \mathrm{H}$ & HGMD & M520R & HGMD & C108Y & HGMD & N635D & HGMD & & & & \\
\hline L250P & HGMD & Y522S & HGMD & L109R & HGMD & N635I & HGMD & & & & \\
\hline L251Q & HGMD & V524G & HGMD & L109P & HGMD & N635K & HGMD & & & & \\
\hline L251P & ClinVar, HGMD & A525T & HGMD & D111V & HGMD & E637D & HGMD & & & & \\
\hline G252D & HGMD & A525V & HGMD & P114S & HGMD & E637G & HGMD & & & & \\
\hline S253C & ClinVar & R539W & ClinVar, HGMD & M124R & HGMD & E637K & ClinVar, HGMD & & & & \\
\hline V254M & ClinVar, HGMD & E543K & HGMD & M124T & HGMD & K638N & HGMD & & & & \\
\hline V254L & HGMD & S546L & HGMD & F129I & HGMD & K638E & HGMD & & & & \\
\hline H258N & HGMD & Q547R & HGMD & D219V* & ClinVar & F640L & ClinVar, HGMD & & & & \\
\hline H258P & HGMD & G548D & HGMD & P334L* & ClinVar & F640V & HGMD & & & & \\
\hline H258R & ClinVar, HGMD & V554A & HGMD & I400N & HGMD & V644L & HGMD & & & & \\
\hline R259C & ClinVar, HGMD & R555C & ClinVar, HGMD & W410S & HGMD & V644F & HGMD & & & & \\
\hline R259L & ClinVar, HGMD & $\mathrm{R} 555 \mathrm{H}$ & HGMD & L413P & HGMD & M645L & HGMD & & & & \\
\hline $\mathrm{R} 259 \mathrm{H}$ & HGMD & R555S & HGMD & Y420C & HGMD & M645V & ClinVar, HGMD & & & & \\
\hline E261Q & HGMD & K557E & HGMD & A422D & HGMD & G648S & HGMD & & & & \\
\hline E261K & HGMD & R561G & HGMD & $\mathrm{A} 422 \mathrm{~T}$ & HGMD & S649P & HGMD & & & & \\
\hline E261V & HGMD & R562M & HGMD & $\mathrm{P} 426 \mathrm{H}$ & HGMD & M651R & HGMD & & & & \\
\hline L262V & HGMD & R562S & HGMD & Y427C & HGMD & S654G & HGMD & & & & \\
\hline T265I & ClinVar, HGMD & L563P & HGMD & Y427H & HGMD & F656C & HGMD & & & & \\
\hline L266P & HGMD & S566F & HGMD & Y427S & HGMD & F656L & HGMD & & & & \\
\hline G269S & ClinVar, HGMD & S566P & HGMD & S428L & HGMD & G657R & HGMD & & & & \\
\hline G269D & ClinVar, HGMD & I567F & HGMD & S428P & HGMD & G657C & HGMD & & & & \\
\hline G272D & ClinVar & I567S & HGMD & A429P & HGMD & G657S & ClinVar, HGMD & & & & \\
\hline G272V & HGMD & I567T & HGMD & P451L & HGMD & I662T & HGMD & & & & \\
\hline L273F & ClinVar, HGMD & G568A & ClinVar, HGMD & D456Y & HGMD & $\mathrm{R} 685 \mathrm{H}$ & HGMD & & & & \\
\hline L273R & HGMD & G568R & HGMD & L457P & HGMD & H687Y & HGMD & & & & \\
\hline F275S & HGMD & $\mathrm{K} 699 \mathrm{E}^{*}$ & HGMD & D460Y & HGMD & $\mathrm{R} 694 \mathrm{H}$ & HGMD & & & & \\
\hline S277L & ClinVar, HGMD & S571L* & HGMD & F463L & HGMD & R696P & HGMD & & & & \\
\hline S277P & HGMD & F573L* & HGMD & D466Y & HGMD & S706C & HGMD & & & & \\
\hline S277W & HGMD & $\mathrm{R}_{583 C^{*}}$ & HGMD & N470D & ClinVar, HGMD & A715V & HGMD & & & & \\
\hline Y278H & HGMD & $R 583 G^{*}$ & HGMD & $\mathrm{T} 473 \mathrm{~N}$ & HGMD & P721L & HGMD & & & & \\
\hline Y281C & HGMD & N586D & HGMD & T473P & HGMD & I728F & HGMD & & & & \\
\hline L282P & HGMD & N586S & HGMD & T474I & ClinVar, HGMD & $\mathrm{R} 744 \mathrm{P}$ & HGMD & & & & \\
\hline A283T & HGMD & T587M & ClinVar, HGMD & Y475C & HGMD & $\mathrm{R} 752 \mathrm{Q}$ & HGMD & & & & \\
\hline АЗ302Т & HGMD & G589D & ClinVar, HGMD & E480V & HGMD & $\mathrm{R} 752 \mathrm{~W}$ & HGMD & & & & \\
\hline $\mathrm{A} 302 \mathrm{~V}$ & HGMD & T587R & HGMD & I489F & HGMD & A753S & HGMD & & & & \\
\hline L303P & HGMD & A590T & HGMD & A490P & HGMD & K757N & HGMD & & & & \\
\hline W304R & HGMD & R591C & HGMD & A490T & ClinVar, HGMD & D767Y & HGMD & & & & \\
\hline
\end{tabular}


TABLE 1 | Continued

\begin{tabular}{|c|c|c|c|c|c|c|c|c|c|c|c|}
\hline \multicolumn{4}{|c|}{ KCNQ1 } & \multicolumn{4}{|c|}{ hERG } & \multicolumn{4}{|c|}{ SCN5A } \\
\hline Mutant & Database & Mutant & Database & Mutant & Database & Mutant & Database & Mutant & Database & Mutant & Database \\
\hline W305R & HGMD & $\mathrm{R} 591 \mathrm{H}$ & ClinVar, HGMD & H492Y & HGMD & V770A & HGMD & & & & \\
\hline W305L & ClinVar, HGMD & R591L & HGMD & Y493C & HGMD & D774Y & HGMD & & & & \\
\hline G306R & ClinVar, HGMD & $\mathrm{R} 594 \mathrm{Q}$ & HGMD & Y493F & HGMD & G785A & HGMD & & & & \\
\hline G306V & HGMD & E596K & HGMD & Y493S & HGMD & G785V & HGMD & & & & \\
\hline V308D & HGMD & L602P & HGMD & W497L & HGMD & G785D & HGMD & & & & \\
\hline T309R & HGMD & $1609 \mathrm{~N}$ & HGMD & D501N & HGMD & E788D & HGMD & & & & \\
\hline Т309| & HGMD & D611Y & HGMD & D501G & ClinVar, HGMD & E788K & HGMD & & & & \\
\hline V310I & HGMD & $\mathrm{G} 635 \mathrm{R}^{\star}$ & HGMD & D501H & HGMD & V795I & HGMD & & & & \\
\hline \multirow[t]{30}{*}{ T311A } & HGMD & & & G522R & HGMD & G800A & ClinVar & & & & \\
\hline & & & & K525N & HGMD & G800E & HGMD & & & & \\
\hline & & & & $\mathrm{R} 528 \mathrm{P}$ & HGMD & G800W & HGMD & & & & \\
\hline & & & & $\mathrm{R} 531 \mathrm{Q}$ & HGMD & M801I & ClinVar & & & & \\
\hline & & & & $\mathrm{R} 534 \mathrm{C}$ & ClinVar, HGMD & D803Y & HGMD & & & & \\
\hline & & & & R534L & HGMD & F805C & HGMD & & & & \\
\hline & & & & R537W & HGMD & F805S & ClinVar, HGMD & & & & \\
\hline & & & & L552S & ClinVar, HGMD & G806E & ClinVar, HGMD & & & & \\
\hline & & & & E544A & HGMD & P815L & HGMD & & & & \\
\hline & & & & A558E & HGMD & G816V & HGMD & & & & \\
\hline & & & & A558P & ClinVar, HGMD & S818L & HGMD & & & & \\
\hline & & & & L559H & HGMD & S818P & HGMD & & & & \\
\hline & & & & A561P & ClinVar, HGMD & S818W & HGMD & & & & \\
\hline & & & & A561T & ClinVar, HGMD & G820E & HGMD & & & & \\
\hline & & & & A561V & HGMD & G820R & ClinVar, HGMD & & & & \\
\hline & & & & H562R & HGMD & V822I & ClinVar & & & & \\
\hline & & & & W563C & HGMD & V822L & HGMD & & & & \\
\hline & & & & W563G & HGMD & V822M & HGMD & & & & \\
\hline & & & & L564P & HGMD & R823W & HGMD & & & & \\
\hline & & & & A565T & HGMD & T826I & ClinVar, HGMD & & & & \\
\hline & & & & C566F & HGMD & R835W & HGMD & & & & \\
\hline & & & & C566S & HGMD & D837N & HGMD & & & & \\
\hline & & & & W568R & HGMD & D837G & HGMD & & & & \\
\hline & & & & W568C & HGMD & $\mathrm{D} 837 \mathrm{H}$ & HGMD & & & & \\
\hline & & & & Y569C & HGMD & D837Y & HGMD & & & & \\
\hline & & & & Y569H & HGMD & V841L & HGMD & & & & \\
\hline & & & & I571L & HGMD & P846T & HGMD & & & & \\
\hline & & & & I571M & HGMD & I858T & HGMD & & & & \\
\hline & & & & G572D & HGMD & $\mathrm{N} 861 \mathrm{H}$ & HGMD & & & & \\
\hline & & & & G572C & HGMD & N861I & ClinVar, HGMD & & & & \\
\hline
\end{tabular}

Curated mutations classified as pathogenic in the ClinVar (Harrison et al., 2016) and HGMD (Stenson et al., 2012) databases. Mutations were cross-referenced to remove any mutations with conflicting classifications. Asterisked mutations are not mapped onto the channel structures presented in Figures 9-11 because those positions are not resolved in the final models. The Excel file for this table can be found as "Table 1" in the Supplementary Material.

mutation, but also cellular quality control responses to these changes.

The VSD of hERG has fewer pathogenic mutations than that of KCNQ1 (Figure 10B), perhaps an indication that the hERG VSD has a higher folding stability. Indeed, when a set of hERG VSD mutations were assessed for membrane insertion efficiency, the majority of mutations were predicted to have no change in the free energy of insertion (Anderson et al., 2014). However, there are a surprising number of disease mutations at the extracellular interface between S1 and S5, specifically at the A422, P426, Y427, S428, H562, W563, C566, and Y569 positions (Figures 10B, C). This interface has not been studied extensively. However, we speculate that it may be important to channel stability by providing an additional contact surface between the VSD and the PD distinct from the S4-S5 linker. Destabilization of this interface may therefore lead to mistrafficking. Mutation Indeed, A422T results in ER retention but was rescued with a pharmacological chaperone (Guo et al., 2012). Other mutations in S1 alter gating properties in addition to trafficking (Anderson et al., 2014; Phan et al., 2017), indicating that gating properties may also be affected. Further studies are needed to determine the impact of mutations in this interface on folding, trafficking, and gating properties of hERG.

A number of mutations are found in the PAS and CNBHD, particularly in the PAS and at the interface between the two domains (Figures 10A, D). The structural stability of both domains impacts trafficking (Akhavan et al., 2005; Harley et al., 2012; Ke et al., 2013; Ke et al., 2014), and several trafficking-defective mutants have been characterized at the PAS/CNBHD interface (F29L, I31S, I42N, Y43C, R56Q, M124R), suggesting that occlusion of a PAS hydrophobic patch from the cytoplasm through interaction with the CNBHD is critical for proper trafficking (Ke et al., 2013). Trafficking defects due to destabilization of the PAS, the $\mathrm{CNBHD}$, or their interaction may thus be a prominent mechanism 
in LQT2. The direct interaction of these two domains has also been implicated in slow deactivation of the channel ( $\mathrm{Ng}$ et al., 2014; Kume et al., 2018), as deactivation defects have been noted in channels with mutations at this interface (Anderson et al., 2014). Deactivation defects may be a secondary source of LQT2. Detailed structural analysis of mutation-induced destabilization of the PAS and the CNBHD as well as studies of cellular responses to PAS/ CNBHD destabilization would be highly informative to our understanding of LQT2. Indeed, similar studies have already revealed differential cellular responses to LQT2 PAS mutations, depending on the severity of PAS destabilization (Foo et al., 2019).

\section{SCN5A}

LQT3 mutations induce channel gain-of-function in SCN5A (Wilde and Amin, 2018; Skinner et al., 2019). Accordingly, mutations that interfere with SCN5A folding and trafficking do not cause LQT3 (Bohnen et al., 2017; Wilde and Amin, 2018). The primary mechanism leading to LQT3 is rather impairment of fast inactivation (Bohnen et al., 2017; Wilde and Amin, 2018). The results of our analysis are consistent with this notion. The site where the S5 and S6 helices of repeats III and IV cross, in the binding pocket for the IFM motif, contains the majority of the mutations curated in this study (Figures 11A, C). These mutations may impact interaction of the IFM motif with other channel structures, hindering fast inactivation and generating a persistent sodium current. For instance, A1326 (A1328 in rSCN5A) and G1329 (G1331 in rSCN5A) make critical backbone interactions to help form a tight hydrophobic pocket for IFM interactions (Jiang et al., 2020). Mutations A1326P and G1329S (Table 1) may thus compromise the stability of this pocket, hindering inactivation. Additionally, S6 mutations surrounding this putative inactivation gate also map to the constriction site of the channel pore, with additional mutations in $S 6_{I}$ and $S 6_{\text {II }}$ (Figure 11D). It has been proposed that binding of the IFM motif causes a rotation in the $\mathrm{S} 6$ helices that closes the pore (Yan et al., 2017b). Therefore mutations in S6 may also alter inactivation by preventing helix rotation. Indeed, residues important to defining the constriction site of the $\mathrm{Na}_{\mathrm{v}} 1.7$ pore cause LQTS upon mutation in SCN5A (L398 and Y1755) (Shen et al., 2019). A few mutations also map to the CTD of SCN5A (Figure 11B), which could alter interactions with the III/IV linker and hinder fast inactivation.

The recent structure of the rat homolog of SCN5A (Jiang et al., 2020) suggests another gain-of-function mechanism, namely that VSD mutant R225P could promote current leak through the VSD (gating pore current). Experimental evidence supporting this mechanism exists for other SCN5A (Moreau et al., 2015) and $\mathrm{Na}_{\mathrm{V}} 1.4$ (Sokolov et al., 2007) VSD mutations.

\section{STRUCTURAL BASIS FOR CHANNEL PHARMACOLOGY}

Pharmacological management of LQTS aims to minimize symptomatic arrhythmia and prevent life-threatening cardiac events. According to consensus guidelines (Zipes et al., 2006; Priori et al., 2013), treatment with $\beta$-blockers is beneficial and effective in the case of LQTS diagnosis, but should be considered only after additional diagnostics for carriers of LQTS mutations. $\beta$-blockers work by inhibiting $\beta$-adrenergic receptors, consequently slowing heart rate and preventing arrhythmias triggered by activation of the sympathetic nervous system (Bohnen et al., 2017). However, the efficacy of $\beta$-blockers is strongly genotype- and trigger-specific (Moss et al., 2000; Schwartz et al., 2001; Bohnen et al., 2017). $\beta$-blockers reduce the risk of exercise-induced arrhythmia by $78 \%$ and $71 \%$ in patients with LQT1 and LQT2, respectively, but neither are protective against cardiac events triggered by emotional arousal or during rest (Kim et al., 2010; Goldenberg et al., 2012). Patients with LQT3 tend to experience the highest breakthrough rate for cardiac events $(10-15 \%)$ with $\beta$-blocker treatment (Giudicessi and Ackerman, 2013). Use of local anesthetic-type antiarrhythmic agents (e.g. mexiletine) for clinical treatment of LQT3, however, can be accompanied by undesirable side effects (Moreno et al., 2011). The need for improved LQTS pharmacotherapy is further supported by the difficulties surrounding hERG modulation, as several clinically-used drugs can cause drug-induced LQTS by blocking hERG and suppressing $\mathrm{I}_{\mathrm{Kr}}$ (Vandenberg et al., 2012; Kalyaanamoorthy and Barakat, 2018). This section summarizes some known pharmacological agents and the modes of interaction with KCNQ1, hERG, and SCN5A. Special focus is dedicated toward unique structural features of hERG that have been implicated in the promiscuity of this channel for pro-arrhythmic drugs.

\section{KCNQ1}

KCNQ1 channels are targeted by several non-selective antiarrhythmic drugs such as quinidine (Balser et al., 1991), amiodarone (Balser et al., 1991; Kamiya et al., 2001), azimilide (Busch et al., 1997), and clofilium (Yang et al., 1997). More specific KCNQ1 blockers include chromanol 293B (Bosch et al., 1998), and the benzodiazepine L-735821 (Lengyel et al., 2001). The binding site of L-735821 has been located to the SF and S6 (Seebohm et al., 2003a).

Benzodiazepine R-L3 was the first KCNQ1 activator to be described. The compound acts to slow the deactivation rate and causes a hyperpolarizing shift in the voltage-dependence of channel activation (Salata et al., 1998). R-L3 appears to bind residues on S5 and S6 lining the membrane-exposed surface of the PD (Seebohm et al., 2003b) rather than to the inner channel cavity. Drug binding outside of the PD cavity was also reported for quinidine (Yang et al., 2013), which occupies a pocket between S6 and the S4-S5 linker and is postulated to elicit an allosteric channel blocking mechanism. More recently, ML277 was identified as a potent KCNQ1 agonist (Mattmann et al., 2012). ML277 has been proposed to bind to a side pocket surrounded by the S2-S3 and S4-S5 linkers on the intracellular side and helices S4 and S6 on the intramembrane lateral side (Xu et al., 2015). Binding of ML277 may selectively alter VSD/PD coupling to stabilize the $\mathrm{AO}$ state relative to the IO state (Hou et al., 2019). 
A
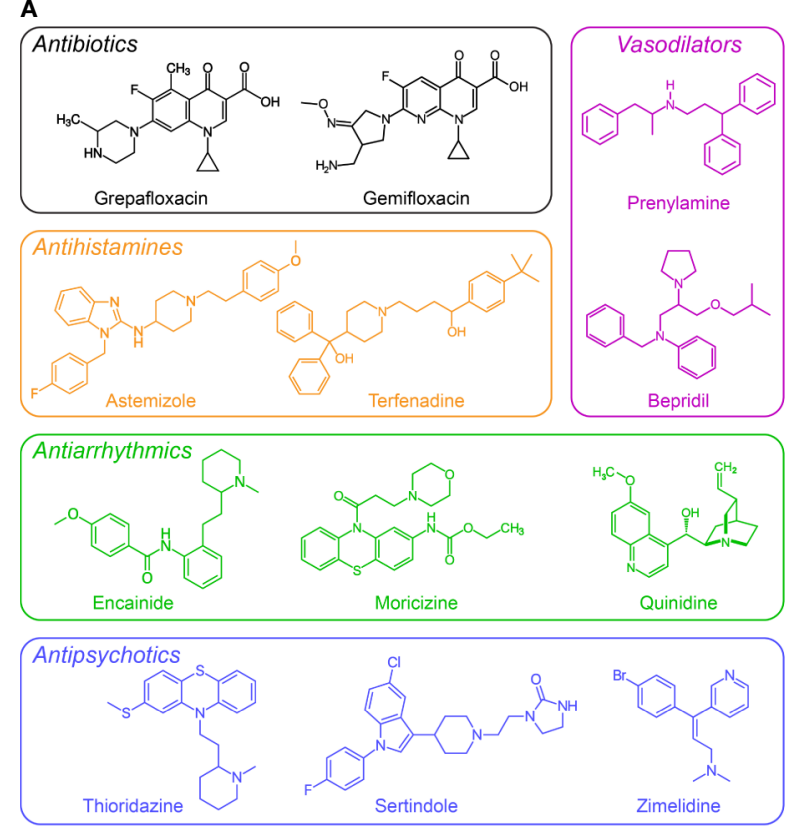

B
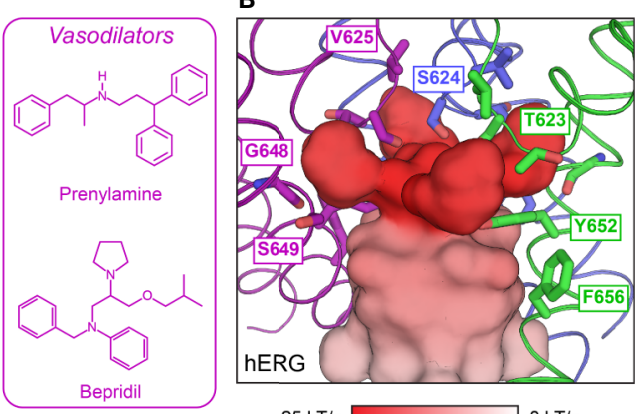

$-25 \mathrm{kT} / \mathrm{e} \square 0 \mathrm{kT} / \mathrm{e}$

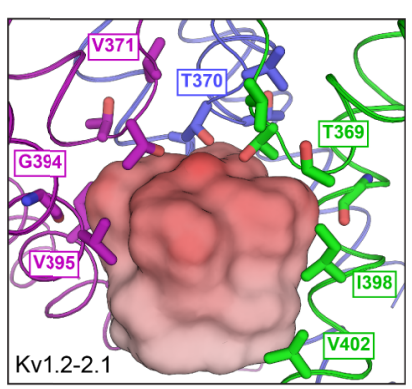

FIGURE 12 | Structural features of the drug-binding cavity of the hERG channel pore. (A) Examples of drugs discontinued by the US FDA because of side effects related to hERG inhibition (adapted from Table $\mathbf{1}$ in (Kalyaanamoorthy and Barakat, 2018)). (B) Internal molecular surface of the central cavity below the SF in hERG (PDB: 5VA1)(Wang and Mackinnon, 2017) (top) and the KV1.2-2.1 chimeric channel (PDB: 2R9R)(Long et al., 2007) (bottom). The surface is colored by the electrostatic potential calculated with the APBS tool in PyMOL (The PyMOL Molecular Graphics System, Version 2.2 Schrödinger, LLC.). Residues related to drug binding by hERG and the corresponding residues in the $\mathrm{K}_{\mathrm{v}} 1.2-2.1$ structure are shown as sticks. This panel is inspired by Figure $\mathbf{5}$ in (Wang and Mackinnon, 2017$)$.

\section{hERG}

hERG channels can be blocked by a wide spectrum of compounds, leading to drug-induced LQTS (Cubeddu, 2016). The list of drugs that inhibit hERG includes, among others, antibiotics (grepafloxacin (Bischoff et al., 2000)), antihistamines (astemizole (Zhou et al., 1999)), anti-arrhythmics (quinidine (Roden et al., 1986), dofetilide (Jurkiewicz and Sanguinetti, 1993)), antipsychotics (sertindole (Rampe et al., 1998)), and gastroprokinetic agents (cisapride (Vitola et al., 1998)) (Figure 12A). Several prescription drugs have been withdrawn from the market because of cardiotoxic side effects triggered by off-target interaction with hERG (Kalyaanamoorthy and Barakat, 2018). In fact, an estimated $15 \%$ of drugs still on the market may cause QT phase prolongation, and $60 \%$ of drugs in development show hERG liability (Vandenberg et al., 2017). It is now required by the US FDA that all compounds considered for advancement to clinical trials be tested for their impact on hERG channel function as part of preclinical toxicity assessments (Center for Drug Evaluation and Research, 2005a; Center for Drug Evaluation and Research, I.C.O.H., 2005b).

Considerable progress has been made in understanding the basic features of drug binding to hERG, and a plausible mechanism for promiscuity of drug binding has emerged. Proarrhythmic drugs commonly bind to the central inner cavity of the PD in hERG. Two pore-lining aromatic residues in S6 (Y652 and F656) are the most crucial for drug binding (Mitcheson et al.,
2000), although additional residues at the end of the pore helix (T623, S624, and V625) contribute to binding for some drugs (Mitcheson et al., 2000), and mutations at F557 in the S5 helix can also affect binding (Saxena et al., 2016). The side chains of the S6 and pore helix residues point towards a putative drug binding site located in the central cavity below the SF. This cavity is slightly narrower in hERG than in the Shaker family $\mathrm{K}_{\mathrm{V}} 1.2-2.1$ chimeric channel (Long et al., 2007). As a consequence, there is a greater negative electrostatic potential in this region (Wang and Mackinnon, 2017), which could enhance drug binding capabilities (Figure 12B). In addition, four lateral hydrophobic pockets, which are not observed in the $\mathrm{K}_{\mathrm{V}} 1.2-2.1$ structure, extend outwards from that central constriction site, generating additional space to trap chemical moieties (Wang and Mackinnon, 2017).

Quantitative structure-activity relationship studies have identified a pharmacophore for drugs that bind to hERG (Cavalli et al., 2002). The pharmacophore consists of three centers of mass (usually aromatic rings) and an amino group that together form a flattened tetrahedron. It has been suggested that the aromatic rings could fit into one or more lateral hydrophobic pockets in hERG and/or bind by pi-pi stacking or hydrophobic interactions with Y652 and F656 (Chen et al., 2002). Likewise, the greater negative electrostatic potential in the central cavity could explain why many drugs that bind hERG, including off-targets, contain a positive charge (Fernandez et al., 2004). Additionally, a number of docking models for drug- 
bound hERG, with and without this pharmacophore, are being generated (Wacker et al., 2017; Helliwell et al., 2018; Negami et al., 2019), making possible further delineation of drug binding modes within this pocket and the structural basis of their affinity for hERG.

The majority of high-affinity drugs that bind hERG off-target preferentially bind to the inactivated state rather the open state, with affinity differences ranging from 2- to 100-fold (Perrin et al., 2008). Interestingly, the related EAG1 channel (Whicher and Mackinnon, 2016) is less sensitive to inhibition by most of hERG-blocking drugs even though they share the same porelining aromatic residues. One major difference between hERG and EAG1 is that EAG1 channels undergo minimal inactivation (Garg et al., 2012) whereas hERG channels undergo rapid and complete inactivation (Vandenberg et al., 2012). The contribution of inactivation to high-affinity binding is further substantiated by the observation that inactivating EAG1-hERG chimeras, containing the upper half of the hERG PD, can bind drugs with almost hERG-like affinity (Herzberg et al., 1998). While cryo-EM structures have been determined for both hERG and EAG1 (Whicher and Mackinnon, 2016; Wang and Mackinnon, 2017), the structural basis of the functional and pharmacological differences requires higher resolution (Wang and Mackinnon, 2017).

\section{SCN5A}

Limited progress has been made in the development of SCN5Atargeted treatment strategies. Classical non-selective sodium channel blockers like the Vaughan-Williams class I antiarrhythmic drugs quinidine, lidocaine, and propafenone have only limited clinical applicability. Ranolazine is a more selective SCN5A blocker (Belardinelli et al., 2006; Zaza et al., 2008) and has been shown to attenuate action potential duration and reduce or prevent intracellular calcium overload in LQT3 models (Wu et al., 2004; Lindegger et al., 2009). Mexiletine is a potent inhibitor of SCN5A and may be beneficial for treatment of LQT3 (Mazzanti et al., 2016), but effectiveness may depend on the baseline QT prolongation of the mutant channel (Li and Zhang, 2018).

Despite the clinical relevance of SCN5A-targeting drugs, the molecular mechanisms of promising and potentially problematic drugs are not well understood at a structural level, hindering drug design. However, key residues for apparent drug binding have been suggested within the sodium channel pore domain. Specifically, mutations of two conserved aromatic residues in S6 $6_{\text {IV }}$, F1760 and Y1767, (Ragsdale et al., 1994; Ragsdale et al., 1996) or other S6 residues in repeats I and III (Yarov-Yarovoy et al., 2001; Yarov-Yarovoy et al., 2002) may affect drug binding. Using fluorinated phenylalanine derivatives incorporated at F1760 (Pless et al., 2011a), a strong cation-pi interaction between F1760 and the protonated amine group in class Ib anti-arrhythmic drugs was observed, defining the molecular basis for inhibition of SCN5A by this group of drugs. Additionally, mutations within the SF region can affect apparent drug binding either by enhancing slow inactivation or forming an alternative access pathway (Sunami et al., 1997; Lee et al., 2001; Tsang et al., 2005). Interactions of anti-arrhythmic and local anesthetic drugs (lidocaine, QX-314, etidocaine, flecainide, and ranolazine) docked into an inactivated state model of SCN5A (Nguyen et al., 2019) revealed several key drug binding sites in the inner pore lumen that can simultaneously accommodate up to two drug molecules. Subsequent MD simulations suggested alternative access mechanisms for drugs into the SCN5A lumen - a hydrophilic pathway through the intracellular gate and a hydrophobic pathway through a membrane-exposed fenestration between repeats III and IV.

The recent structure of rat $\mathrm{Na}_{\mathrm{v}} 1.5$ (Jiang et al., 2020) has provided insight into the binding mode of the class Ic antiarrhythmic drug flecainide, which has higher affinity for the open conformation of SCN5A (Ramos and O'leary M, 2004). Flecainide binds in the central cavity on the intracellular side of the SF. The piperidine ring of flecainide lies across the top of the central cavity with the positively-charged nitrogen pointing towards the exit of the SF and its hydrophobic edge extending towards the phenyl ring of F1762 (F1760 in human $\left.\mathrm{Na}_{\mathrm{v}} 1.5\right)$. The trifluoroethoxy tails of flecainide extend into fenestrations formed between the pore helices of repeats I and II as well as repeats II and III, through which the central cavity is accessible from the lipid bilayer. These fenestrations represent possible entry points for flecainide and similar class Ic drugs.

\section{FUTURE DIRECTIONS}

The growing number of structural and functional studies of KCNQ1, hERG, and SCN5A have provided new insight into channel molecular physiology and channel dysfunction in congenital LQTS. This further motivates the study of fundamental biophysical mechanisms in ion channels. One of these mechanisms, electromechanical coupling, remains enigmatic due to a dearth of experimental resting state structures and robust methods to identify allosteric pathways, which cannot easily be discerned from ion channel structures resolved in a single conformation. However, interaction energy and MD dynamical network analysis in the Shaker $\mathrm{K}^{+}$channel (Fernandez-Marino et al., 2018) has suggested that movements in the VSD and PD are linked by two distinct pathways - a canonical pathway through the S4-S5 linker and a hitherto unknown noncanonical pathway involving contacts at the interface between S4 and S5. Such work may provide a useful alternative approach to explore electromechanical coupling in detail.

Another major effort involves the experimental characterization of hundreds of previously uncharacterized variants of unknown significance (VUS) and LQTS mutations in KCNQ1, hERG, and SCN5A. Widespread use of whole genome and exome sequencing has provided a nearly complete catalog of common sequence variations in protein-coding genes (Lek et al., 2016), although rare sequence variants continue to be discovered. For many LQTS mutations, the molecular mechanisms responsible for impaired channel function remain poorly understood. Such mutations may cause potassium channel loss-of-function or dysfunction by promoting misfolding, mistrafficking, aggregation, or improper gating of the channel protein, as discussed above. Continued experimental testing will be required to complete functional annotation of KCNQ1, hERG, and SCN5A variants 
and to elucidate how these mutations lead to channel dysfunction. In this regard, new techniques such as high-throughput patch clamp recording (Vanoye et al., 2018; Toh et al., 2020), which allows rapid screening of ion channel variants found in patients, and the use of induced-pluripotent stem cell-derived cardiomyocytes (Moretti et al., 2010; Ma et al., 2013; Terrenoire et al., 2013; Jouni et al., 2015) developed from cells of healthy or mutation-carrying patients, represent helpful tools for large-scale examination of ion channel variants.

Finally, experimentally-informed bioinformatics and modeling approaches are being developed to predict variant pathogenicity (Kroncke et al., 2015; Li et al., 2017; Kroncke et al., 2019). These computational algorithms can help to decrypt newly discovered VUS for which there is not enough data to determine pathogenicity. Decrypting VUS can inform medical practice and has the potential to improve LQTS therapy, both for the prevention of cardiac events in susceptible patients and as a basis for avoiding unneeded treatment in healthy patients. With improved understanding of the molecular pathophysiology and the role of mutations associated with distinct LQTS subtypes, the ultimate hope is that identifying a patient's genotype will lead to more specific treatment considerations and the delivery of optimal care.

\section{AUTHOR CONTRIBUTIONS}

$\mathrm{KB}$ and $\mathrm{GK}$ wrote the primary draft of this article, with edits by $\mathrm{KB}, \mathrm{GK}, \mathrm{JM}, \mathrm{CV}, \mathrm{AG}$, and CS. KB and GK contributed equally to this manuscript.

\section{FUNDING}

This work was supported by US NIH grant RO1 HL122010. GK was supported by a postdoctoral fellowship from the American Heart Association (18POST34080422) and KB was supported by $\mathrm{NIH}$ training grant T32 GM008320.

\section{SUPPLEMENTARY MATERIAL}

The Supplementary Material for this article can be found online at: https://www.frontiersin.org/articles/10.3389/fphar.2020.00550 /full\#supplementary-material

TABLE 1 | LQTS mutations in KCNQ1, hERG, and SCN5A.

\section{REFERENCES}

Abbott, G. W., Sesti, F., Splawski, I., Buck, M. E., Lehmann, M. H., Timothy, K. W., et al. (1999). MiRP1 forms IKr potassium channels with HERG and is associated with cardiac arrhythmia. Cell 97, 175-187. doi: 10.1016/S0092-8674(00)80728-X

Abbott, G. W., Xu, X., and Roepke, T. K. (2007). Impact of ancillary subunits on ventricular repolarization. J. Electrocardiol. 40, S42-S46. doi: 10.1016/ j.jelectrocard.2007.05.021

Abbott, G. W. (2014). Biology of the KCNQ1 Potassium Channel. New J. Sci. 2014, 26. doi: $10.1155 / 2014 / 237431$

Ackerman, M. J. (2005). Genotype-phenotype relationships in congenital long QT syndrome. J. Electrocardiol. 38, 64-68. doi: 10.1016/j.jelectrocard.2005.06.018

Adler, A., Novelli, V., Amin, A. S., Abiusi, E., Care, M., Nannenberg, E. A., et al. (2020). An International, Multicentered, Evidence-Based Reappraisal of Genes Reported to Cause Congenital Long QT Syndrome. Circulation 141, 418-428. doi: 10.1161/CIRCULATIONAHA.119.043132

Aggarwal, S. K., and Mackinnon, R. (1996). Contribution of the S4 segment to gating charge in the Shaker K+ channel. Neuron 16, 1169-1177. doi: 10.1016/ S0896-6273(00)80143-9

Ahern, C. A., Payandeh, J., Bosmans, F., and Chanda, B. (2016). The hitchhiker's guide to the voltage-gated sodium channel galaxy. J. Gen. Physiol. 147, 1-24. doi: 10.1085/jgp.201511492

Akhavan, A., Atanasiu, R., Noguchi, T., Han, W., Holder, N., and Shrier, A. (2005). Identification of the cyclic-nucleotide-binding domain as a conserved determinant of ion-channel cell-surface localization. J. Cell Sci. 118, 28032812. doi: $10.1242 /$ jcs. 02423

Anantharam, A., and Abbott, G. W. (2005). Does hERG coassemble with a beta subunit? Evidence for roles of MinK and MiRP1. Novartis Found Symp 266, 100-112discussion 112-107, 155-108. doi: 10.1002/047002142X.ch9

Anderson, C. L., Kuzmicki, C. E., Childs, R. R., Hintz, C. J., Delisle, B. P., and January, C. T. (2014). Large-scale mutational analysis of Kv11.1 reveals molecular insights into type 2 long QT syndrome. Nat. Commun. 5, 5535. doi: $10.1038 /$ ncomms6535

Apaja, P. M., Foo, B., Okiyoneda, T., Valinsky, W. C., Barriere, H., Atanasiu, R., et al. (2013). Ubiquitination-dependent quality control of hERG $\mathrm{K}+$ channel with acquired and inherited conformational defect at the plasma membrane. Mol. Biol. Cell 24, 3787-3804. doi: 10.1091/mbc.e13-07-0417

Balser, J. R., Bennett, P. B., Hondeghem, L. M., and Roden, D. M. (1991) Suppression of Time-Dependent Outward Current in Guinea-Pig Ventricular Myocytes - Actions of Quinidine and Amiodarone. Circ. Res. 69, 519-529. doi: 10.1161/01.RES.69.2.519

Barhanin, J., Lesage, F., Guillemare, E., Fink, M., Lazdunski, M., and Romey, G. (1996). K(v)LQT1 and IsK (minK) proteins associate to form the I-Ks cardiac potassium current. Nature 384, 78-80. doi: 10.1038/384078a0

Baroni, D., Picco, C., Barbieri, R., and Moran, O. (2014). Antisense-mediated posttranscriptional silencing of SCN1B gene modulates sodium channel functional expression. Biol. Cell 106, 13-29. doi: 10.1111/boc.201300040

Barros, F., Dominguez, P., and De La Pena, P. (2018). Relative positioning of Kv11.1 (hERG) $\mathrm{K}(+)$ channel cytoplasmic domain-located fluorescent tags toward the plasma membrane. Sci. Rep. 8, 15494. doi: 10.1038/s41598-018-33492-x

Barros, F., Pardo, L. A., Dominguez, P., Sierra, L. M., and De La Pena, P. (2019). New Structures and Gating of Voltage-Dependent Potassium (Kv) Channels and Their Relatives: A Multi-Domain and Dynamic Question. Int. J. Mol. Sci. 20, pii: E248. doi: 10.3390/ijms20020248

Barro-Soria, R., Rebolledo, S., Liin, S. I., Perez, M. E., Sampson, K. J., Kass, R. S., et al. (2014). KCNE1 divides the voltage sensor movement in KCNQ1/KCNE1 channels into two steps. Nat. Commun. 5, 3750. doi: 10.1038/ncomms4750

Barro-Soria, R., Ramentol, R., Liin, S. I., Perez, M. E., Kass, R. S., and Larsson, H. P. (2017). KCNE1 and KCNE3 modulate KCNQ1 channels by affecting different gating transitions. Proc. Natl. Acad. Sci. U. S. A 114, E7367-E7376. doi: 10.1073/pnas.1710335114

Belardinelli, L., Shryock, J. C., and Fraser, H. (2006). Inhibition of the late sodium current as a potential cardioprotective principle: effects of the late sodium current inhibitor ranolazine. Heart 92 Suppl 4, iv6-iv14. doi: 10.1136/ hrt.2005.078790

Bernardo-Seisdedos, G., Nunez, E., Gomis-Perez, C., Malo, C., Villarroel, A., and Millet, O. (2018). Structural basis and energy landscape for the $\mathrm{Ca}(2+)$ gating and calmodulation of the Kv7.2 K(+) channel. Proc. Natl. Acad. Sci. U. S. A 115, 2395-2400. doi: 10.1073/pnas.1800235115

Bezanilla, F. (2005). Voltage-gated ion channels. IEEE Trans. Nanobiosci. 4, 34-48. doi: 10.1109/TNB.2004.842463

Bhate, M. P., Wylie, B. J., Tian, L., and Mcdermott, A. E. (2010). Conformational dynamics in the selectivity filter of KcsA in response to potassium ion concentration. J. Mol. Biol. 401, 155-166. doi: 10.1016/j.jmb.2010.06.031 
Bian, J., Cui, J., and Mcdonald, T. V. (2001). HERG K(+) channel activity is regulated by changes in phosphatidyl inositol 4,5-bisphosphate. Circ. Res. 89, 1168-1176. doi: 10.1161/hh2401.101375

Bian, J. S., Kagan, A., and Mcdonald, T. V. (2004). Molecular analysis of PIP2 regulation of HERG and IKr. Am. J. Physiol. Heart Circ. Physiol. 287, H2154H2163. doi: 10.1152/ajpheart.00120.2004

Bidaud, I., and Lory, P. (2011). Hallmarks of the channelopathies associated with L-type calcium channels: a focus on the Timothy mutations in $\mathrm{Ca}(\mathrm{v}) 1.2$ channels. Biochimie 93, 2080-2086. doi: 10.1016/j.biochi.2011.05.015

Bischoff, U., Schmidt, C., Netzer, R., and Pongs, O. (2000). Effects of fluoroquinolones on HERG currents. Eur. J. Pharmacol. 406, 341-343. doi: 10.1016/S0014-2999(00)00693-2

Bohnen, M. S., Peng, G., Robey, S. H., Terrenoire, C., Iyer, V., Sampson, K. J., et al. (2017). Molecular Pathophysiology of Congenital Long QT Syndrome. Physiol. Rev. 97, 89-134. doi: 10.1152/physrev.00008.2016

Boland, L. M., and Drzewiecki, M. M. (2008). Polyunsaturated fatty acid modulation of voltage-gated ion channels. Cell Biochem. Biophys. 52, 59-84. doi: 10.1007/s12013-008-9027-2

Bosch, R. F., Gaspo, R., Busch, A. E., Lang, H. J., Li, G. R., and Nattel, S. (1998). Effects of the chromanol 293B, a selective blocker of the slow, component of the delayed rectifier $\mathrm{K}+$ current, on repolarization in human and guinea pig ventricular myocytes. Cardiovasc. Res. 38, 441-450. doi: 10.1016/S0008-6363(98)00021-2

Boulet, I. R., Raes, A. L., Ottschytsch, N., and Snyders, D. J. (2006). Functional effects of a KCNQ1 mutation associated with the long QT syndrome. Cardiovasc. Res. 70, 466-474. doi: 10.1016/j.cardiores.2006.02.006

Boulet, I. R., Labro, A. J., Raes, A. L., and Snyders, D. J. (2007). Role of the S6 Cterminus in KCNQ1 channel gating. J. Physiol. 585, 325-337. doi: 10.1113/ jphysiol.2007.145813

Brackenbury, W. J., and Isom, L. L. (2011). Na Channel beta Subunits: Overachievers of the Ion Channel Family. Front. Pharmacol. 2, 53. doi: 10.3389/fphar.2011.00053

Burgess, D. E., Bartos, D. C., Reloj, A. R., Campbell, K. S., Johnson, J. N., Tester, D. J., et al. (2012). High-risk long QT syndrome mutations in the Kv7.1 (KCNQ1) pore disrupt the molecular basis for rapid $\mathrm{K}(+)$ permeation. Biochemistry 51, 9076-9085. doi: 10.1021/bi3009449

Busch, A. E., Busch, G. L., Ford, E., Suessbrich, H., Lang, H. J., Greger, R., et al. (1997). The role of the IsK protein in the specific pharmacological properties of the IKs channel complex. Br. J. Pharmacol. 122, 187-189. doi: 10.1038/ sj.bjp. 0701434

Calhoun, J. D., and Isom, L. L. (2014). The role of non-pore-forming beta subunits in physiology and pathophysiology of voltage-gated sodium channels. Handb. Exp. Pharmacol. 221, 51-89. doi: 10.1007/978-3-642-41588-3_4

Capes, D. L., Goldschen-Ohm, M. P., Arcisio-Miranda, M., Bezanilla, F., and Chanda, B. (2013). Domain IV voltage-sensor movement is both sufficient and rate limiting for fast inactivation in sodium channels. J. Gen. Physiol. 142, 101112. doi: 10.1085/jgp.201310998

Catterall, W. A. (1986). Molecular properties of voltage-sensitive sodium channels. Annu. Rev. Biochem. 55, 953-985. doi: 10.1146/annurev.bi.55.070186.004513

Cavalli, A., Poluzzi, E., De Ponti, F., and Recanatini, M. (2002). Toward a pharmacophore for drugs inducing the long QT syndrome: insights from a CoMFA study of HERG K(+) channel blockers. J. Med. Chem. 45, 3844-3853. doi: $10.1021 / \mathrm{jm} 0208875$

Chakrabarti, N., Ing, C., Payandeh, J., Zheng, N., Catterall, W. A., and Pomes, R. (2013). Catalysis of $\mathrm{Na}+$ permeation in the bacterial sodium channel $\mathrm{Na}(\mathrm{V}) \mathrm{Ab}$. Proc. Natl. Acad. Sci. U. S. A 110, 11331-11336. doi: 10.1073/pnas.1309452110

Chan, P. J., Osteen, J. D., Xiong, D., Bohnen, M. S., Doshi, D., Sampson, K. J., et al. (2012). Characterization of KCNQ1 atrial fibrillation mutations reveals distinct dependence on KCNE1. J. Gen. Physiol. 139, 135-144. doi: 10.1085/ jgp.201110672

Chanda, B., and Bezanilla, F. (2002). Tracking voltage-dependent conformational changes in skeletal muscle sodium channel during activation. J. Gen. Physiol. 120, 629-645. doi: 10.1085/jgp.20028679

Chang, A., Abderemane-Ali, F., Hura, G. L., Rossen, N. D., Gate, R. E., and Minor, D. L.Jr. (2018). A Calmodulin C-Lobe Ca(2+)-Dependent Switch Governs Kv7 Channel Function. Neuron 97, 836-852 e836. doi: 10.1016/j.neuron.2018.01.035

Chen, J., Seebohm, G., and Sanguinetti, M. C. (2002). Position of aromatic residues in the S6 domain, not inactivation, dictates cisapride sensitivity of HERG and eag potassium channels. Proc. Natl. Acad. Sci. U. S. A 99, 12461-12466. doi: 10.1073/pnas.192367299

Chen, J., Weber, M., Um, S. Y., Walsh, C. A., Tang, Y., and Mcdonald, T. V. (2011). A dual mechanism for I(Ks) current reduction by the pathogenic mutation KCNQ1-S277L. Pacing Clin. Electrophysiol. 34, 1652-1664. doi: 10.1111/j.1540-8159.2011.03190.x

Chen, L., Zhang, Q., Qiu, Y., Li, Z., Chen, Z., Jiang, H., et al. (2015). Migration of PIP2 lipids on voltage-gated potassium channel surface influences channel deactivation. Sci. Rep. 5, 15079. doi: 10.1038/srep15079

Chen, X. M., Guo, K., Li, H., Lu, Q. F., Yang, C., Yu, Y., et al. (2019). A novel mutation KCNQ1p.Thr312del is responsible for long QT syndrome type 1. Heart Vessels 34, 177-188. doi: 10.1007/s00380-018-1223-4

Cheng, Y. M., and Claydon, T. W. (2012). Voltage-dependent gating of HERG potassium channels. Front. Pharmacol. 3, 83. doi: 10.3389/fphar.2012.00083

Cheng, J. H., and Kodama, I. (2004). Two components of delayed rectifier K+ current in heart: molecular basis, functional diversity, and contribution to repolarization. Acta Pharmacol. Sin. 25, 137-145.

Choveau, F. S., and Shapiro, M. S. (2012). Regions of KCNQ K(+) channels controlling functional expression. Front. Physiol. 3, 397. doi: 10.3389/ fphys.2012.00397

Chung, D. Y., Chan, P. J., Bankston, J. R., Yang, L., Liu, G., Marx, S. O., et al. (2009). Location of KCNE1 relative to KCNQ1 in the I(KS) potassium channel by disulfide cross-linking of substituted cysteines. Proc. Natl. Acad. Sci. U. S. A 106, 743-748. doi: 10.1073/pnas.0811897106

Clairfeuille, T., Cloake, A., Infield, D. T., Llongueras, J. P., Arthur, C. P., Li, Z. R., et al. (2019). Structural basis of alpha-scorpion toxin action on Nav channels. Science 363, pii: eaav8573. doi: 10.1126/science.aav8573

Codding, S. J., and Trudeau, M. C. (2019). The hERG potassium channel intrinsic ligand regulates N- and C-terminal interactions and channel closure. J. Gen. Physiol. 151, 478-488. doi: 10.1085/jgp.201812129

Cubeddu, L. X. (2016). Drug-induced Inhibition and Trafficking Disruption of ion Channels: Pathogenesis of QT Abnormalities and Drug-induced Fatal Arrhythmias. Curr. Cardiol. Rev. 12, 141-154. doi: 10.2174/ 1573403X12666160301120217

Cuello, L. G., Jogini, V., Cortes, D. M., and Perozo, E. (2010). Structural mechanism of C-type inactivation in $\mathrm{K}(+)$ channels. Nature 466, 203-208. doi: 10.1038/nature09153

Cui, J. (2016). Voltage-Dependent Gating: Novel Insights from KCNQ1 Channels. Biophys. J. 110, 14-25. doi: 10.1016/j.bpj.2015.11.023

De La Pena, P., Alonso-Ron, C., Machin, A., Fernandez-Trillo, J., Carretero, L., Dominguez, P., et al. (2011). Demonstration of physical proximity between the $\mathrm{N}$ terminus and the S4-S5 linker of the human ether-a-go-go-related gene (hERG) potassium channel. J. Biol. Chem. 286, 19065-19075. doi: 10.1074/ jbc.M111.238899

Dehghani-Samani, A., Madreseh-Ghahfarokhi, S., and Dehghani-Samani, A. (2019). Mutations of Voltage-Gated Ionic Channels and Risk of Severe Cardiac Arrhythmias. Acta Cardiol. Sin. 35, 99-110. doi: 10.6515/ ACS.201903_35(2).20181028A

Detta, N., Frisso, G., and Salvatore, F. (2015). The multi-faceted aspects of the complex cardiac Nav1.5 protein in membrane function and pathophysiology. Biochim. Biophys. Acta 1854, 1502-1509. doi: 10.1016/j.bbapap.2015.07.009

Dhar Malhotra, J., Chen, C., Rivolta, I., Abriel, H., Malhotra, R., Mattei, L. N., et al. (2001). Characterization of sodium channel alpha- and beta-subunits in rat and mouse cardiac myocytes. Circulation 103, 1303-1310. doi: 10.1161/ 01.CIR.103.9.1303

Doolan, G. K., Panchal, R. G., Fonnes, E. L., Clarke, A. L., Williams, D. A., and Petrou, S. (2002). Fatty acid augmentation of the cardiac slowly activating delayed rectifier current (IKs) is conferred by hminK. FASEB J. 16, 1662-1664. doi: 10.1096/fj.02-0084fje

Doyle, D. A., Morais Cabral, J., Pfuetzner, R. A., Kuo, A., Gulbis, J. M., Cohen, S. L., et al. (1998). The structure of the potassium channel: molecular basis of $\mathrm{K}+$ conduction and selectivity. Science 280, 69-77. doi: 10.1126/ science. 280.5360 .6923

Earle, N., Crawford, J., Smith, W., Hayes, I., Shelling, A., Hood, M., et al. (2013). Community detection of long QT syndrome with a clinical registry: an alternative to ECG screening programs? Heart Rhythm. 10, 233-238. doi: 10.1016/j.hrthm.2012.10.043 
Eckey, K., Wrobel, E., Strutz-Seebohm, N., Pott, L., Schmitt, N., and Seebohm, G. (2014). Novel Kv7.1-phosphatidylinositol 4,5-bisphosphate interaction sites uncovered by charge neutralization scanning. J. Biol. Chem. 289, 22749-22758. doi: 10.1074/jbc.M114.589796

Fan, J. S., Jiang, M., Dun, W., Mcdonald, T. V., and Tseng, G. N. (1999). Effects of outer mouth mutations on hERG channel function: a comparison with similar mutations in the Shaker channel. Biophys. J. 76, 3128-3140. doi: 10.1016/ S0006-3495(99)77464-3

Favre, I., Moczydlowski, E., and Schild, L. (1996). On the structural basis for ionic selectivity among $\mathrm{Na}+\mathrm{K}+$, and $\mathrm{Ca} 2+$ in the voltage-gated sodium channel. Biophys. J. 71, 3110-3125. doi: 10.1016/S0006-3495(96)79505-X

Ferrer, T., Cordero-Morales, J. F., Arias, M., Ficker, E., Medovoy, D., Perozo, E., et al (2011). Molecular coupling in the human ether-a-go-go-related gene-1 (hERG1) K+ channel inactivation pathway. J. Biol. Chem. 286 (45), 3909139099. doi: 10.1074/jbc.M111.292060

Fernandez, D., Ghanta, A., Kauffman, G. W., and Sanguinetti, M. C. (2004). Physicochemical features of the HERG channel drug binding site. J. Biol. Chem. 279, 10120-10127. doi: 10.1074/jbc.M310683200

Fernandez-Marino, A. I., Harpole, T. J., Oelstrom, K., Delemotte, L., and Chanda, B. (2018). Gating interaction maps reveal a noncanonical electromechanical coupling mode in the Shaker K(+) channel. Nat. Struct. Mol. Biol. 25, 320-326. doi: 10.1038/s41594-018-0047-3

Ficker, E., Jarolimek, W., and Brown, A. M. (2001). Molecular determinants of inactivation and dofetilide block in ether a-go-go (EAG) channels and EAGrelated K(+) channels. Mol. Pharmacol. 60, 1343-1348. doi: 10.1124/mol.60.6.1343

Ficker, E., Dennis, A. T., Wang, L., and Brown, A. M. (2003). Role of the cytosolic chaperones Hsp70 and Hsp90 in maturation of the cardiac potassium channel HERG. Circ. Res. 92, e87-100. doi: 10.1161/01.RES.0000079028.31393.15

Foo, B., Barbier, C., Guo, K., Vasantharuban, J., Lukacs, G. L., and Shrier, A. (2019). Mutation-specific peripheral and ER quality control of hERG channel cell-surface expression. Sci. Rep. 9, 6066. doi: 10.1038/s41598-019-42331-6

Franqueza, L., Lin, M., Shen, J., Splawski, I., Keating, M. T., and Sanguinetti, M. C. (1999). Long QT syndrome-associated mutations in the S4-S5 linker of KvLQT1 potassium channels modify gating and interaction with $\mathrm{minK}$ subunits. J. Biol. Chem. 274, 21063-21070. doi: 10.1074/jbc.274.30.21063

Gabelli, S. B., Boto, A., Kuhns, V. H., Bianchet, M. A., Farinelli, F., Aripirala, S., et al. (2014). Regulation of the NaV1.5 cytoplasmic domain by calmodulin. Nat. Commun. 5, 5126. doi: 10.1038/ncomms6126

Gardill, B. R., Rivera-Acevedo, R. E., Tung, C. C., and Van Petegem, F. (2019). Crystal structures of $\mathrm{Ca}(2+)$-calmodulin bound to $\mathrm{NaV}$ C-terminal regions suggest role for EF-hand domain in binding and inactivation. Proc. Natl. Acad. Sci. U. S. A 116, 10763-10772. doi: 10.1073/pnas.1818618116

Garg, V., Sachse, F. B., and Sanguinetti, M. C. (2012). Tuning of EAG K(+) channel inactivation: molecular determinants of amplification by mutations and a small molecule. J. Gen. Physiol. 140, 307-324. doi: 10.1085/ jgp.201210826

George, A. L. Jr. (2013). Molecular and genetic basis of sudden cardiac death. J. Clin. Invest. 123, 75-83. doi: 10.1172/JCI62928

Ghosh, S., Nunziato, D. A., and Pitt, G. S. (2006). KCNQ1 assembly and function is blocked by long-QT syndrome mutations that disrupt interaction with calmodulin. Circ. Res. 98, 1048-1054. doi: 10.1161/01.RES.0000218863.44140.f2

Ghovanloo, M. R., Aimar, K., Ghadiry-Tavi, R., Yu, A., and Ruben, P. C. (2016). Physiology and Pathophysiology of Sodium Channel Inactivation. Curr. Top. Membr. 78, 479-509. doi: 10.1016/bs.ctm.2016.04.001

Giudicessi, J. R., and Ackerman, M. J. (2013). Genotype- and phenotype-guided management of congenital long QT syndrome. Curr. Probl. Cardiol. 38, 417455. doi: 10.1016/j.cpcardiol.2013.08.001

Gofman, Y., Shats, S., Attali, B., Haliloglu, T., and Ben-Tal, N. (2012). How does KCNE1 regulate the Kv7.1 potassium channel? Model-structure, mutations, and dynamics of the Kv7.1-KCNE1 complex. Structure 20, 1343-1352. doi: 10.1016/j.str.2012.05.016

Goldenberg, I., Thottathil, P., Lopes, C. M., Moss, A. J., Mcnitt, S., O-Uchi, J., et al. (2012). Trigger-specific ion-channel mechanisms, risk factors, and response to therapy in type 1 long QT syndrome. Heart Rhythm. 9, 49-56. doi: 10.1016/ j.hrthm.2011.08.020

Goldschen-Ohm, M. P., Capes, D. L., Oelstrom, K. M., and Chanda, B. (2013). Multiple pore conformations driven by asynchronous movements of voltage sensors in a eukaryotic sodium channel. Nat. Commun. 4, 1350. doi: 10.1038/ ncomms 2356

Gordon, E., Panaghie, G., Deng, L., Bee, K. J., Roepke, T. K., Krogh-Madsen, T., et al. (2008). A KCNE2 mutation in a patient with cardiac arrhythmia induced by auditory stimuli and serum electrolyte imbalance. Cardiovasc. Res. 77, 98106. doi: $10.1093 / \mathrm{cvr} / \mathrm{cvm} 030$

Grant, A. O. (2009). Cardiac ion channels. Circ. Arrhythm Electrophysiol. 2, 185194. doi: 10.1161/CIRCEP.108.789081

Guo, J., Zhang, X., Hu, Z., Zhuang, Z., Zhu, Z., Chen, Z., et al. (2012). A422T mutation in HERG potassium channel retained in ER is rescurable by pharmacologic or molecular chaperones. Biochem. Biophys. Res. Commun. 422, 305-310. doi: 10.1016/j.bbrc.2012.04.153

Gustina, A. S., and Trudeau, M. C. (2011). hERG potassium channel gating is mediated by N- and C-terminal region interactions. J. Gen. Physiol. 137, 315325. doi: 10.1085/jgp.201010582

Haitin, Y., and Attali, B. (2008). The C-terminus of Kv7 channels: a multifunctional module. J. Physiol. 586, 1803-1810. doi: 10.1113/ jphysiol.2007.149187

Hancox, J. C., Whittaker, D. G., Du, C., Stuart, A. G., and Zhang, H. (2018). Emerging therapeutic targets in the short QT syndrome. Expert Opin. Ther. Targets 22, 439-451. doi: 10.1080/14728222.2018.1470621

Harley, C. A., Jesus, C. S., Carvalho, R., Brito, R. M., and Morais-Cabral, J. H. (2012). Changes in channel trafficking and protein stability caused by LQT2 mutations in the PAS domain of the HERG channel. PloS One 7, e32654. doi: 10.1371/journal.pone.0032654

Harrison, S. M., Riggs, E. R., Maglott, D. R., Lee, J. M., Azzariti, D. R., Niehaus, A., et al. (2016). Using ClinVar as a Resource to Support Variant Interpretation. Curr. Protoc. Hum. Genet. 898 16, 11-18 16 23. doi: 10.1002/0471142905.hg0816s89

Heijman, J., Spatjens, R. L., Seyen, S. R., Lentink, V., Kuijpers, H. J., Boulet, I. R., et al. (2012). Dominant-negative control of cAMP-dependent IKs upregulation in human long-QT syndrome type 1. Circ. Res. 110, 211-219. doi: 10.1161/ CIRCRESAHA.111.249482

Heitzmann, D., Grahammer, F., Von Hahn, T., Schmitt-Graff, A., Romeo, E., Nitschke, R., et al. (2004). Heteromeric KCNE2/KCNQ1 potassium channels in the luminal membrane of gastric parietal cells. J. Physiol. 561, 547-557. doi: 10.1113/jphysiol.2004.075168

Helliwell, M. V., Zhang, Y., El Harchi, A., Du, C., Hancox, J. C., and Dempsey, C. E. (2018). Structural implications of hERG $\mathrm{K}(+)$ channel block by a highaffinity minimally structured blocker. J. Biol. Chem. 293, 7040-7057. doi: 10.1074/jbc.RA117.000363

Henry, J. T., and Crosson, S. (2011). Ligand-binding PAS domains in a genomic, cellular, and structural context. Annu. Rev. Microbiol. 65, 261-286. doi: 10.1146/annurev-micro-121809-151631

Herzberg, I. M., Trudeau, M. C., and Robertson, G. A. (1998). Transfer of rapid inactivation and sensitivity to the class III antiarrhythmic drug E-4031 from HERG to M-eag channels. J. Physiol. 511 ( Pt 1), 3-14. doi: 10.1111/j.14697793.1998.003bi.x

Herzik, M. A.Jr., Fraser, J. S., and Lander, G. C. (2019). A Multi-model Approach to Assessing Local and Global Cryo-EM Map Quality. Structure 27344-358, e343. doi: 10.1016/j.str.2018.10.003

Hille, B. (1971). The hydration of sodium ions crossing the nerve membrane. Proc. Natl. Acad. Sci. U. S. A 68, 280-282. doi: 10.1073/pnas.68.2.280

Hoosien, M., Ahearn, M. E., Myerburg, R. J., Pham, T. V., Miller, T. E., Smets, M. J., et al. (2013). Dysfunctional potassium channel subunit interaction as a novel mechanism of long QT syndrome. Heart Rhythm. 10, 728-737. doi: 10.1016/ j.hrthm.2012.12.033

Hoshi, T., and Armstrong, C. M. (2013). C-type inactivation of voltage-gated K+ channels: pore constriction or dilation? J. Gen. Physiol. 141, 151-160. doi: 10.1085/jgp.201210888

Hosseini, S. M., Kim, R., Udupa, S., Costain, G., Jobling, R., Liston, E., et al. (2018). Reappraisal of Reported Genes for Sudden Arrhythmic Death: Evidence-Based Evaluation of Gene Validity for Brugada Syndrome. Circulation 138, 11951205. doi: 10.1161/CIRCULATIONAHA.118.035070

Hosseini, S. M. (2018). A useful hand mnemonic to demonstrate ion channel gating. Adv. Physiol. Educ. 42, 321-323. doi: 10.1152/advan.00080.2017

Hou, P., Eldstrom, J., Shi, J., Zhong, L., Mcfarland, K., Gao, Y., et al. (2017). Inactivation of KCNQ1 potassium channels reveals dynamic coupling between 
voltage sensing and pore opening. Nat. Commun. 8, 1730. doi: 10.1038/s41467017-01911-8

Hou, P., Shi, J., White, K. M., Gao, Y., and Cui, J. (2019). ML277 specifically enhances the fully activated open state of KCNQ1 by modulating VSD-pore coupling. Elife 8, e48576. doi: 10.7554/eLife.48576

Hou, P., Kang, P. W., Kongmeneck, A. D., Yang, N. D., Liu, Y., Shi, J., et al. (2020). Two-stage electro-mechanical coupling of a KV channel in voltage-dependent activation. Nat. Commun. 11, 676. doi: 10.1038/s41467-020-14406-w

Hovey, L., Fowler, C. A., Mahling, R., Lin, Z., Miller, M. S., Marx, D. C., et al. (2017). Calcium triggers reversal of calmodulin on nested anti-parallel sites in the IQ motif of the neuronal voltage-dependent sodium channel NaV1.2. Biophys. Chem. 224, 1-19. doi: 10.1016/j.bpc.2017.02.006

Howard, R. J., Clark, K. A., Holton, J. M., and Minor, D. L.Jr. (2007). Structural insight into KCNQ (Kv7) channel assembly and channelopathy. Neuron 53, 663-675. doi: 10.1016/j.neuron.2007.02.010

Hu, D., Barajas-Martinez, H., Burashnikov, E., Springer, M., Wu, Y., Varro, A., et al. (2009). A mutation in the beta 3 subunit of the cardiac sodium channel associated with Brugada ECG phenotype. Circ. Cardiovasc. Genet. 2, 270-278. doi: 10.1161/CIRCGENETICS.108.829192

Hu, D., Barajas-Martinez, H., Medeiros-Domingo, A., Crotti, L., Veltmann, C., Schimpf, R., et al. (2012). A novel rare variant in SCN1Bb linked to Brugada syndrome and SIDS by combined modulation of $\mathrm{Na}(\mathrm{v}) 1.5$ and $\mathrm{K}(\mathrm{v}) 4.3$ channel currents. Heart Rhythm. 9, 760-769. doi: 10.1016/j.hrthm.2011.12.006

Huang, H., Kuenze, G., Smith, J. A., Taylor, K. C., Duran, A. M., Hadziselimovic, A., et al. (2018). Mechanisms of KCNQ1 channel dysfunction in long QT syndrome involving voltage sensor domain mutations. Sci. Adv. 4, eaar2631. doi: 10.1126/sciadv.aar2631

Center for Drug Evaluation and Research. (2005a). "E14: clinical evaluation of QT/ QTc interval prolongation and proarrhythmic potential for non-arrhythmic drugs", (ed.) U.S.F.a.D. Administration.).

Center for Drug Evaluation and Research. (2005b). "S7B: The non-clinical evaluation of the potential for delayed ventricular depolarization (QT interval prolongation) by human pharmaceruticals", (ed.) U.S.F.a.D. Administration.)

Ikrar, T., Hanawa, H., Watanabe, H., Okada, S., Aizawa, Y., Ramadan, M. M., et al. (2008). A double-point mutation in the selectivity filter site of the KCNQ1 potassium channel results in a severe phenotype, LQT1, of long QT syndrome. J. Cardiovasc. Electrophysiol. 19, 541-549. doi: 10.1111/j.1540-8167.2007.01076.x

Iqbal, S. M., Aufy, M., Shabbir, W., and Lemmens-Gruber, R. (2018). Identification of phosphorylation sites and binding pockets for modulation of NaV 1.5 channel by Fyn tyrosine kinase. FEBS J. 285, 2520-2530. doi: 10.1111/febs.14496

Isbilen, B., Fraser, S. P., and Djamgoz, M. B. (2006). Docosahexaenoic acid (omega-3) blocks voltage-gated sodium channel activity and migration of MDA-MB-231 human breast cancer cells. Int. J. Biochem. Cell Biol. 38, 21732182. doi: $10.1016 / j . b i o c e l .2006 .06 .014$

Jiang, Y., Ruta, V., Chen, J., Lee, A., and Mackinnon, R. (2003). The principle of gating charge movement in a voltage-dependent $\mathrm{K}+$ channel. Nature $423,42-$ 48. doi: 10.1038 /nature 01581

Jiang, D., Shi, H., Tonggu, L., Gamal El-Din, T. M., Lenaeus, M. J., Zhao, Y., et al. (2020). Structure of the Cardiac Sodium Channel. Cell 180, 122-134 e110. doi: 10.1016/j.cell.2019.11.041

Johnson, C. N., Potet, F., Thompson, M. K., Kroncke, B. M., Glazer, A. M., Voehler, M. W., et al. (2018). A Mechanism of Calmodulin Modulation of the Human Cardiac Sodium Channel. Structure 26, 683-694 e683. doi: 10.1016/j.str.2018.03.005

Jouni, M., Si-Tayeb, K., Es-Salah-Lamoureux, Z., Latypova, X., Champon, B., Caillaud, A., et al. (2015). Toward Personalized Medicine: Using Cardiomyocytes Differentiated From Urine-Derived Pluripotent Stem Cells to Recapitulate Electrophysiological Characteristics of Type 2 Long QT Syndrome. J. Am. Heart Assoc. 4, e002159. doi: 10.1161/JAHA.115.002159

Jurkiewicz, N. K., and Sanguinetti, M. C. (1993). Rate-dependent prolongation of cardiac action potentials by a methanesulfonanilide class III antiarrhythmic agent. Specific block of rapidly activating delayed rectifier $\mathrm{K}+$ current by dofetilide. Circ. Res. 72, 75-83. doi: 10.1161/01.RES.72.1.75

Kalyaanamoorthy, S., and Barakat, K. H. (2018). Development of Safe Drugs: The hERG Challenge. Med. Res. Rev. 38, 525-555. doi: 10.1002/med.21445

Kamiya, K., Nishiyama, A., Yasui, K., Hojo, M., Sanguinetti, M. C., and Kodama, I. (2001). Short- and long-term effects of amiodarone on the two components of cardiac delayed rectifier K(+) current. Circulation 103, 1317-1324. doi: 10.1161/01.CIR.103.9.1317

Kang, J. X., Xiao, Y. F., and Leaf, A. (1995). ). Free, long-chain, polyunsaturated fatty acids reduce membrane electrical excitability in neonatal rat cardiac myocytes. Proc. Natl. Acad. Sci. U. S. A 92, 3997-4001. doi: 10.1073/ pnas.92.9.3997

Kang, J. X., Li, Y., and Leaf, A. (1997). Regulation of sodium channel gene expression by class I antiarrhythmic drugs and $\mathrm{n}-3$ polyunsaturated fatty acids in cultured neonatal rat cardiac myocytes. Proc. Natl. Acad. Sci. U. S. A 94, 2724-2728. doi: 10.1073/pnas.94.6.2724

Kang, C., Tian, C., Sonnichsen, F. D., Smith, J. A., Meiler, J., George, A. L.Jr., et al. (2008). Structure of KCNE1 and implications for how it modulates the KCNQ1 potassium channel. Biochemistry 47, 7999-8006. doi: 10.1021/bi800875q

Kanki, H., Kupershmidt, S., Yang, T., Wells, S., and Roden, D. M. (2004). A structural requirement for processing the cardiac K+ channel KCNQ1. J. Biol. Chem. 279, 33976-33983. doi: 10.1074/jbc.M404539200

Kapa, S., Tester, D. J., Salisbury, B. A., Harris-Kerr, C., Pungliya, M. S., Alders, M., et al. (2009). Genetic testing for long-QT syndrome: distinguishing pathogenic mutations from benign variants. Circulation 120, 1752-1760. doi: 10.1161/ CIRCULATIONAHA.109.863076

Kasimova, M. A., Zaydman, M. A., Cui, J., and Tarek, M. (2015). PIP2-dependent coupling is prominent in Kv7.1 due to weakened interactions between S4-S5 and S6. Sci. Rep. 5, 7474-7474. doi: 10.1038/srep07474

Ke, Y., Ng, C. A., Hunter, M. J., Mann, S. A., Heide, J., Hill, A. P., et al. (2013). Trafficking defects in PAS domain mutant Kv11.1 channels: roles of reduced domain stability and altered domain-domain interactions. Biochem. J. 454, 6977. doi: 10.1042/BJ20130328

Ke, Y., Hunter, M. J., Ng, C. A., Perry, M. D., and Vandenberg, J. I. (2014). Role of the cytoplasmic N-terminal Cap and Per-Arnt-Sim (PAS) domain in trafficking and stabilization of Kv11.1 channels. J. Biol. Chem. 289, 1378213791. doi: 10.1074/jbc.M113.531277

Khan, H. M., Tieleman, P. D., and Noskov, S. Y. (2020). "Refinement of Highresolution Cryo-EM Structure of hERG: What Can We Expect?", in The Biophysical Society 64th Annual Meeting (San Diego: Cell Press).

Kim, J., Ghosh, S., Liu, H., Tateyama, M., Kass, R. S., and Pitt, G. S. (2004). Calmodulin mediates Ca2+ sensitivity of sodium channels. J. Biol. Chem. 279, 45004-45012. doi: 10.1074/jbc.M407286200

Kim, J. A., Lopes, C. M., Moss, A. J., Mcnitt, S., Barsheshet, A., Robinson, J. L., et al. (2010). Trigger-specific risk factors and response to therapy in long QT syndrome type 2. Heart Rhythm. 7, 1797-1805. doi: 10.1016/ j.hrthm.2010.09.011

Kleber Ag, R. Y. (2004). Basic mechanisms of cardiac impulse propagation and associated arrhythmias. Physiol. Rev. 84, 431-488. doi: 10.1152/ physrev.00025.2003

Kroncke Bm, E. A. (2019). Protein structure aids in predicting functional perturbation of missense variants of SCN5A and KCNQ1. Comput. Struct. Biotechnol. J. 17,206-214. doi: 10.1016/j.csbj.2019.01.008

Kroncke, B. M., Vanoye, C. G., Meiler, J., George, A. L. Jr., and Sanders, C. R. (2015). Personalized biochemistry and biophysics. Biochemistry 54, 25512559. doi: 10.1021/acs.biochem.5b00189

Kroncke, B. M., Van Horn, W. D., Smith, J., Kang, C., Welch, R. C., Song, Y., et al. (2016). Structural basis for KCNE3 modulation of potassium recycling in epithelia. Sci. Adv. 2, e1501228. doi: 10.1126/sciadv.1501228

Kroncke, B. M., Mendenhall, J., Smith, D. K., Sanders, C. R., Capra, J. A., George, A. L., et al. (2019). Protein structure aids predicting functional perturbation of missense variants in SCN5A and KCNQ1. Comput. Struct. Biotechnol. J. 17, 206-214. doi: 10.1016/j.csbj.2019.01.008

Kuenze, G., Duran, A. M., Woods, H., Brewer, K. R., Mcdonald, E. F., Vanoye, C. G., et al. (2019). Upgraded molecular models of the human KCNQ1 potassium channel. PloS One 14, e0220415. doi: 10.1371/journal.pone.0220415

Kume, S., Shimomura, T., Tateyama, M., and Kubo, Y. (2018). Two mutations at different positions in the CNBH domain of the hERG channel accelerate deactivation and impair the interaction with the EAG domain. J. Physiol. 596, 4629-4650. doi: 10.1113/JP276208

Labro, A. J., and Snyders, D. J. (2012). Being flexible: the voltage-controllable activation gate of kv channels. Front. Pharmacol. 3, 168. doi: 10.3389/ fphar.2012.00168 
Labro, A. J., Boulet, I. R., Choveau, F. S., Mayeur, E., Bruyns, T., Loussouarn, G., et al. (2011). The S4-S5 linker of KCNQ1 channels forms a structural scaffold with the S6 segment controlling gate closure. J. Biol. Chem. 286, 717-725. doi: 10.1074/jbc.M110.146977

Lee, P. J., Sunami, A., and Fozzard, H. A. (2001). Cardiac-specific external paths for lidocaine, defined by isoform-specific residues, accelerate recovery from use-dependent block. Circ. Res. 89, 1014-1021. doi: 10.1161/hh2301.100002

Leifert, W. R., Mcmurchie, E. J., and Saint, D. A. (1999). Inhibition of cardiac sodium currents in adult rat myocytes by $n-3$ polyunsaturated fatty acids. $J$. Physiol. 520 (Pt 3), 671-679. doi: 10.1111/j.1469-7793.1999.00671.x

Lek, M., Karczewski, K. J., Minikel, E. V., Samocha, K. E., Banks, E., Fennell, T., et al. (2016). Analysis of protein-coding genetic variation in 60,706 humans. Nature 536, 285-291. doi: 10.1038/nature19057

Lemaillet, G., Walker, B., and Lambert, S. (2003). Identification of a conserved ankyrin-binding motif in the family of sodium channel alpha subunits. J. Biol. Chem. 278, 27333-27339. doi: 10.1074/jbc.M303327200

Lengyel, C., Iost, N., Virag, L., Varro, A., Lathrop, D. A., and Papp, J. G. (2001). Pharmacological block of the slow component of the outward delayed rectifier current (I(Ks)) fails to lengthen rabbit ventricular muscle QT(c) and action potential duration. Br. J. Pharmacol. 132, 101-110. doi: 10.1038/sj.bjp.0703777

Li, G., and Zhang, L. (2018). The role of mexiletine in the management of long QT syndrome. J. Electrocardiol. 51, 1061-1065. doi: 10.1016/j.jelectrocard.2018.08.035

Li, P., Liu, H., Lai, C., Sun, P., Zeng, W., Wu, F., et al. (2014). Differential modulations of KCNQ1 by auxiliary proteins KCNE1 and KCNE2. Sci. Rep. 4, 4973. doi: $10.1038 /$ srep 04973

Li, B., Mendenhall, J. L., Kroncke, B. M., Taylor, K. C., Huang, H., Smith, D. K., et al. (2017). Predicting the Functional Impact of KCNQ1 Variants of Unknown Significance. Circ. Cardiovasc. Genet. 10, pii: e001754. doi: 10.1161/CIRCGENETICS.117.001754

Li, M. C. H., O'brien, T. J., Todaro, M., and Powell, K. L. (2019). Acquired cardiac channelopathies in epilepsy: Evidence, mechanisms, and clinical significance. Epilepsia 60, 1753-1767. doi: 10.1111/epi.16301

Liin, S. I., Silvera Ejneby, M., Barro-Soria, R., Skarsfeldt, M. A., Larsson, J. E., Starck Harlin, F., et al. (2015). Polyunsaturated fatty acid analogs act antiarrhythmically on the cardiac IKs channel. Proc. Natl. Acad. Sci. U. S. A 112, 5714-5719. doi: 10.1073/pnas.1503488112

Liin, S. I., Yazdi, S., Ramentol, R., Barro-Soria, R., and Larsson, H. P. (2018). Mechanisms Underlying the Dual Effect of Polyunsaturated Fatty Acid Analogs on Kv7.1. Cell Rep. 24, 2908-2918. doi: 10.1016/j.celrep.2018.08.031

Lin, X., Liu, N., Lu, J., Zhang, J., Anumonwo, J. M., Isom, L. L., et al. (2011). Subcellular heterogeneity of sodium current properties in adult cardiac ventricular myocytes. Heart Rhythm. 8, 1923-1930. doi: 10.1016/ j.hrthm.2011.07.016

Lindegger, N., Hagen, B. M., Marks, A. R., Lederer, W. J., and Kass, R. S. (2009). Diastolic transient inward current in long QT syndrome type 3 is caused by Ca2+ overload and inhibited by ranolazine. J. Mol. Cell Cardiol. 47, 326-334. doi: 10.1016/j.yjmcc.2009.04.003

Liu, D. W., and Antzelevitch, C. (1995). Characteristics of the delayed rectifier current (IKr and IKs) in canine ventricular epicardial, midmyocardial, and endocardial myocytes. A weaker IKs contributes to the longer action potential of the M cell. Circ. Res. 76, 351-365. doi: 10.1161/01.RES.76.3.351

Liu, C. J., Dib-Hajj, S. D., Renganathan, M., Cummins, T. R., and Waxman, S. G. (2003). Modulation of the cardiac sodium channel Nav1.5 by fibroblast growth factor homologous factor 1B. J. Biol. Chem. 278, 1029-1036. doi: 10.1074/jbc.M207074200

Long, S. B., Campbell, E. B., and Mackinnon, R. (2005). Crystal structure of a mammalian voltage-dependent Shaker family K+ channel. Science 309, 897903. doi: 10.1126/science. 1116269

Long, S. B., Tao, X., Campbell, E. B., and Mackinnon, R. (2007). Atomic structure of a voltage-dependent $\mathrm{K}+$ channel in a lipid membrane-like environment. Nature 450, 376-382. doi: 10.1038/nature06265

Loots, E., and Isacoff, E. Y. (1998). Protein rearrangements underlying slow inactivation of the Shaker K+ channel. J. Gen. Physiol. 112, 377-389. doi: 10.1085/jgp.112.4.377

Lorinczi, E., Gomez-Posada, J. C., De La Pena, P., Tomczak, A. P., FernandezTrillo, J., Leipscher, U., et al. (2015). Voltage-dependent gating of KCNH potassium channels lacking a covalent link between voltage-sensing and pore domains. Nat. Commun. 6, 6672. doi: 10.1038/ncomms7672
Loussouarn, G., Park, K. H., Bellocq, C., Baro, I., Charpentier, F., and Escande, D. (2003). Phosphatidylinositol-4,5-bisphosphate, PIP2, controls KCNQ1/ KCNE1 voltage-gated potassium channels: a functional homology between voltage-gated and inward rectifier K+ channels. EMBO J. 22, 5412-5421. doi: $10.1093 / \mathrm{emboj} / \mathrm{cdg} 526$

Lu, Z., Klem, A. M., and Ramu, Y. (2001). Ion conduction pore is conserved among potassium channels. Nature 413 (6858), 809-813. doi: 10.1038/ 35101535

Lu, Z., Klem, A. M., and Ramu,, Y. (2002). Coupling between voltage sensors and activation gate in voltage-gated K+ channels. J. Gen. Physiol. 120 (5), 663-676. doi: 10.1085 /jgp.20028696

Ma, L. J., Ohmert, I., and Vardanyan, V. (2011). Allosteric features of KCNQ1 gating revealed by alanine scanning mutagenesis. Biophys. J. 100, 885-894. doi: 10.1016/j.bpj.2010.12.3726

Ma, D., Wei, H., Zhao, Y., Lu, J., Li, G., Sahib, N. B., et al. (2013). Modeling type 3 long QT syndrome with cardiomyocytes derived from patient-specific induced pluripotent stem cells. Int. J. Cardiol. 168, 5277-5286. doi: 10.1016/ j.ijcard.2013.08.015

Makara, M. A., Curran, J., Little, S. C., Musa, H., Polina, I., Smith, S. A., et al. (2014). Ankyrin-G coordinates intercalated disc signaling platform to regulate cardiac excitability in vivo. Circ. Res. 115, 929-938. doi: 10.1161/ CIRCRESAHA.115.305154

Makita, N., Bennett, P. B., and George, A. L.Jr. (1996). Molecular determinants of beta 1 subunit-induced gating modulation in voltage-dependent $\mathrm{Na}+$ channels. J. Neurosci. 16, 7117-7127. doi: 10.1523/JNEUROSCI.16-22-07117.1996

Malhotra, J. D., Thyagarajan, V., Chen, C., and Isom, L. L. (2004). Tyrosinephosphorylated and nonphosphorylated sodium channel betal subunits are differentially localized in cardiac myocytes. J. Biol. Chem. 279, 40748-40754. doi: 10.1074/jbc.M407243200

Marionneau, C., Lichti, C. F., Lindenbaum, P., Charpentier, F., Nerbonne, J. M., Townsend, R. R., et al. (2012). Mass spectrometry-based identification of native cardiac Nav1.5 channel alpha subunit phosphorylation sites. J. Proteome Res. 11, 5994-6007. doi: 10.1021/pr300702c

Mattmann, M. E., Yu, H., Lin, Z., Xu, K., Huang, X., Long, S., et al. (2012). Identification of (R)-N-(4-(4-methoxyphenyl)thiazol-2-yl)-1-tosylpiperidine2-carboxamide, ML277, as a novel, potent and selective K(v)7.1 (KCNQ1) potassium channel activator. Bioorg. Med. Chem. Lett. 22, 5936-5941. doi: 10.1016/j.bmcl.2012.07.060

Mazhari, R., Greenstein, J. L., Winslow, R. L., Marban, E., and Nuss, H. B. (2001). Molecular interactions between two long-QT syndrome gene products, HERG and KCNE2, rationalized by in vitro and in silico analysis. Circ. Res. 89, 33-38. doi: 10.1161/hh1301.093633

Mazzanti, A., Maragna, R., Faragli, A., Monteforte, N., Bloise, R., Memmi, M., et al. (2016). Gene-Specific Therapy With Mexiletine Reduces Arrhythmic Events in Patients With Long QT Syndrome Type 3. J. Am. Coll. Cardiol. 67, 1053-1058. doi: 10.1016/j.jacc.2015.12.033

Mcdonald, T. V., Yu, Z., Ming, Z., Palma, E., Meyers, M. B., Wang, K. W., et al. (1997). A minK-HERG complex regulates the cardiac potassium current $\mathrm{I}(\mathrm{Kr})$. Nature 388, 289-292. doi: 10.1038/40882

Meadows, L., Malhotra, J. D., Stetzer, A., Isom, L. L., and Ragsdale, D. S. (2001). The intracellular segment of the sodium channel beta 1 subunit is required for its efficient association with the channel alpha subunit. J. Neurochem. 76, 18711878. doi: 10.1046/j.1471-4159.2001.00192.x

Medeiros-Domingo, A., Kaku, T., Tester, D. J., Iturralde-Torres, P., Itty, A., Ye, B., et al. (2007). SCN4B-encoded sodium channel beta4 subunit in congenital long-QT syndrome. Circulation 116, 134-142. doi: 10.1161/ CIRCULATIONAHA.106.659086

Melman, Y. F., Domenech, A., De La Luna, S., and Mcdonald, T. V. (2001). Structural determinants of KvLQT1 control by the KCNE family of proteins. J. Biol. Chem. 276, 6439-6444. doi: 10.1074/jbc.M010713200

Melman, Y. F., Krumerman, A., and Mcdonald, T. V. (2002). A single transmembrane site in the KCNE-encoded proteins controls the specificity of KvLQT1 channel gating. J. Biol. Chem. 277, 25187-25194. doi: 10.1074/ jbc.M200564200

Melman, Y. F., Um, S. Y., Krumerman, A., Kagan, A., and Mcdonald, T. V. (2004). KCNE1 binds to the KCNQ1 pore to regulate potassium channel activity. Neuron 42, 927-937. doi: 10.1016/j.neuron.2004.06.001 
Meza, U., Beqollari, D., and Bannister, R. A. (2018). Molecular mechanisms and physiological relevance of RGK proteins in the heart. Acta Physiol. (Oxf) 222, e13016. doi: 10.1111/apha.13016

Mitcheson, J. S., Chen, J., Lin, M., Culberson, C., and Sanguinetti, M. C. (2000). A structural basis for drug-induced long QT syndrome. Proc. Natl. Acad. Sci. U. S. A 97, 12329-12333. doi: 10.1073/pnas.210244497

Mohler, P. J., Rivolta, I., Napolitano, C., Lemaillet, G., Lambert, S., Priori, S. G., et al. (2004). Nav1.5 E1053K mutation causing Brugada syndrome blocks binding to ankyrin-G and expression of Nav1.5 on the surface of cardiomyocytes. Proc. Natl. Acad. Sci. U. S. A 101, 17533-17538. doi: 10.1073/pnas.0403711101

Morais Cabral, J. H., Lee, A., Cohen, S. L., Chait, B. T., Li, M., and Mackinnon, R. (1998). Crystal structure and functional analysis of the HERG potassium channel N terminus: a eukaryotic PAS domain. Cell 95, 649-655. doi: 10.1016/ S0092-8674(00)81635-9

Morais-Cabral, J. H., Zhou, Y., and Mackinnon, R. (2001). Energetic optimization of ion conduction rate by the K+ selectivity filter. Nature 414, 37-42. doi: $10.1038 / 35102000$

Moreau, A., Gosselin-Badaroudine, P., Delemotte, L., Klein, M. L., and Chahine, M. (2015). Gating pore currents are defects in common with two Nav1.5 mutations in patients with mixed arrhythmias and dilated cardiomyopathy. J. Gen. Physiol. 145, 93-106. doi: 10.1085/jgp.201411304

Moreno, J. D., Zhu, Z. I., Yang, P. C., Bankston, J. R., Jeng, M. T., Kang, C., et al. (2011). A computational model to predict the effects of class I anti-arrhythmic drugs on ventricular rhythms. Sci. Transl. Med. 3, 98ra83. doi: 10.1126/ scitranslmed.3002588

Moretti, A., Bellin, M., Welling, A., Jung, C. B., Lam, J. T., Bott-Flugel, L., et al. (2010). Patient-specific induced pluripotent stem-cell models for long-QT syndrome. N. Engl. J. Med. 363, 1397-1409. doi: 10.1056/NEJMoa0908679

Moss, A. J., and Kass, R. S. (2005). Long QT syndrome: from channels to cardiac arrhythmias. J. Clin. Invest. 115, 2018-2024. doi: 10.1172/JCI25537

Moss, A. J., Zareba, W., Hall, W. J., Schwartz, P. J., Crampton, R. S., Benhorin, J., et al. (2000). Effectiveness and limitations of beta-blocker therapy in congenital long-QT syndrome. Circulation 101, 616-623. doi: 10.1161/01.CIR.101.6.616

Moss, A. J., Shimizu, W., Wilde, A. A., Towbin, J. A., Zareba, W., Robinson, J. L., et al. (2007). Clinical aspects of type-1 long-QT syndrome by location, coding type, and biophysical function of mutations involving the KCNQ1 gene. Circulation 115, 2481-2489. doi: 10.1161/CIRCULATIONAHA.106.665406

Mousavi Nik, A., Gharaie, S., and Jeong Kim, H. (2015). Cellular mechanisms of mutations in Kv7.1: auditory functions in Jervell and Lange-Nielsen syndrome vs. Romano-Ward syndrome. Front. Cell Neurosci. 9, 32. doi: 10.3389/ fncel.2015.00032

Musa, H., Kline, C. F., Sturm, A. C., Murphy, N., Adelman, S., Wang, C., et al. (2015). SCN5A variant that blocks fibroblast growth factor homologous factor regulation causes human arrhythmia. Proc. Natl. Acad. Sci. U. S. A. 112, 1252812533. doi: $10.1073 /$ pnas. 1516430112

Muskett, F. W., Thouta, S., Thomson, S. J., Bowen, A., Stansfeld, P. J., and Mitcheson, J. S. (2011). Mechanistic insight into human ether-a-go-go-related gene (hERG) $\mathrm{K}+$ channel deactivation gating from the solution structure of the EAG domain. J. Biol. Chem. 286, 6184-6191. doi: 10.1074/jbc.M110.199364

Nakajima, T., Kaneko, Y., Dharmawan, T., and Kurabayashi, M. (2019). Role of the voltage sensor module in Nav domain IV on fast inactivation in sodium channelopathies: The implication of closed-state inactivation. Channels (Austin) 13, 331-343. doi: 10.1080/19336950.2019.1649521

Nakajo, K., and Kubo, Y. (2007). KCNE1 and KCNE3 stabilize and/or slow voltage sensing S4 segment of KCNQ1 channel. J. Gen. Physiol. 130, 269-281. doi: 10.1085/jgp.200709805

Nakajo, K., and Kubo, Y. (2014). Steric hindrance between S4 and S5 of the KCNQ1/KCNE1 channel hampers pore opening. Nat. Commun. 5, 4100-4100. doi: $10.1038 /$ ncomms5100

Naylor, C. E., Bagneris, C., Decaen, P. G., Sula, A., Scaglione, A., Clapham, D. E., et al. (2016). Molecular basis of ion permeability in a voltage-gated sodium channel. EMBO J. 35, 820-830. doi: 10.15252/embj.201593285

Negami, T., Araki, M., Okuno, Y., and Terada, T. (2019). Calculation of absolute binding free energies between the hERG channel and structurally diverse drugs. Sci. Rep. 9, 16586. doi: 10.1038/s41598-019-53120-6

Nerbonne, J. M., and Kass, R. S. (2005). Molecular physiology of cardiac repolarization. Physiol. Rev. 85, 1205-1253. doi: 10.1152/physrev.00002.2005
Ng, C. A., Phan, K., Hill, A. P., Vandenberg, J. I., and Perry, M. D. (2014). Multiple interactions between cytoplasmic domains regulate slow deactivation of Kv11.1 channels. J. Biol. Chem. 289, 25822-25832. doi: 10.1074/jbc.M114.558379

Nguyen, P. T., Demarco, K. R., Vorobyov, I., Clancy, C. E., and Yarov-Yarovoy, V. (2019). Structural basis for antiarrhythmic drug interactions with the human cardiac sodium channel. Proc. Natl. Acad. Sci. U. S. A 116, 2945-2954. doi: 10.1073/pnas.1817446116

O'malley, H. A., and Isom, L. L. (2015). Sodium channel beta subunits: emerging targets in channelopathies. Annu. Rev. Physiol. 77, 481-504. doi: 10.1146/ annurev-physiol-021014-071846

Olesen, M. S., Jespersen, T., Nielsen, J. B., Liang, B., Moller, D. V., Hedley, P., et al. (2011). Mutations in sodium channel beta-subunit SCN3B are associated with early-onset lone atrial fibrillation. Cardiovasc. Res. 89, 786-793. doi: 10.1093/ $\mathrm{cvr} / \mathrm{cvq} 348$

Osteen, J. D., Gonzalez, C., Sampson, K. J., Iyer, V., Rebolledo, S., Larsson, H. P., et al. (2010). KCNE1 alters the voltage sensor movements necessary to open the KCNQ1 channel gate. Proc. Natl. Acad. Sci. U. S. A 107, 22710-22715. doi: 10.1073/pnas. 1016300108

Osteen, J. D., Barro-Soria, R., Robey, S., Sampson, K. J., Kass, R. S., and Larsson, H. P. (2012). Allosteric gating mechanism underlies the flexible gating of KCNQ1 potassium channels. Proc. Natl. Acad. Sci. U. S. A 109, 7103-7108. doi: 10.1073/ pnas.1201582109

Pan, X., Li, Z., Zhou, Q., Shen, H., Wu, K., Huang, X., et al. (2018). Structure of the human voltage-gated sodium channel Nav1.4 in complex with beta1. Science 362, pii: eaau2486. doi: $10.1126 /$ science.aau2486.

Pan, X., Li, Z., Huang, X., Huang, G., Gao, S., Shen, H., et al. (2019). Molecular basis for pore blockade of human $\mathrm{Na}(+)$ channel $\mathrm{Nav1} .2$ by the mu-conotoxin KIIIA. Science 363, 1309-1313. doi: 10.1126/science.aaw2999

Panaghie, G., and Abbott, G. W. (2007). The role of S4 charges in voltagedependent and voltage-independent KCNQ1 potassium channel complexes. J. Gen. Physiol. 129, 121-133. doi: 10.1085/jgp.200609612

Panaghie, G., Tai, K.-K., and Abbott, G. W. (2006). Interaction of KCNE subunits with the KCNQ1 K+ channel pore. J. Physiol. 570, 455-467. doi: 10.1113/ jphysiol.2005.100644

Park, K. H., Piron, J., Dahimene, S., Merot, J., Baro, I., Escande, D., et al. (2005). Impaired KCNQ1-KCNE1 and phosphatidylinositol-4,5-bisphosphate interaction underlies the long QT syndrome. Circ. Res. 96, 730-739. doi: 10.1161/01.RES.0000161451.04649.a8

Perrin, M. J., Kuchel, P. W., Campbell, T. J., and Vandenberg, J. I. (2008). Drug binding to the inactivated state is necessary but not sufficient for high-affinity binding to human ether-a-go-go-related gene channels. Mol. Pharmacol. 74, 1443-1452. doi: 10.1124/mol.108.049056

Perry, M. D., Wong, S., Ng, C. A., and Vandenberg, J. I. (2013a). Hydrophobic interactions between the voltage sensor and pore mediate inactivation in Kv11.1 channels. J. Gen. Physiol. 142 (3), 275-288. doi: 10.1085/jgp.201310975

Perry, M. D., Wong, S., Ng, C. A., and Vandenberg, J. I. (2013b). Pore helices play a dynamic role as integrators of domain motion during Kv11.1 channel inactivation gating. J. Biol. Chem. 288 (16), 11482-11491. doi: 10.1074/jbc.M113.461442

Perry, M. D., Ng, C. A., Mann, S. A., Sadrieh, A., Imtiaz, M., Hill, A. P., et al. (2015). Getting to the heart of hERG K(+) channel gating. J. Physiol. 593, 25752585. doi: $10.1113 /$ JP270095

Phan, K., Ng, C. A., David, E., Shishmarev, D., Kuchel, P. W., Vandenberg, J. I., et al. (2017). The S1 helix critically regulates the finely tuned gating of Kv11.1 channels. J. Biol. Chem. 292, 7688-7705. doi: 10.1074/jbc.M117.779298

Pless, S. A., Galpin, J. D., Frankel, A., and Ahern, C. A. (2011a). Molecular basis for class Ib anti-arrhythmic inhibition of cardiac sodium channels. Nat. Commun. 2, 351. doi: 10.1038/ncomms1351

Pless, S. A., Galpin, J. D., Niciforovic, A. P., and Ahern, C. A. (2011b). Contributions of counter-charge in a potassium channel voltage-sensor domain. Nat. Chem. Biol. 7, 617-623. doi: 10.1038/nchembio.622

Poulsen, K. L., Hotait, M., Calloe, K., Klaerke, D. A., Rebeiz, A., Nemer, G., et al. (2015). The Mutation P.T613a in the Pore Helix of the Kv 11.1 Potassium Channel is Associated with Long QT Syndrome. Pacing Clin. Electrophysiol. 38, 1304-1309. doi: 10.1111/pace.12693

Preston, P., Wartosch, L., Gunzel, D., Fromm, M., Kongsuphol, P., Ousingsawat, J., et al. (2010). Disruption of the K+ channel beta-subunit KCNE3 reveals an important role in intestinal and tracheal Cl- transport. J. Biol. Chem. 285, 7165-7175. doi: 10.1074/jbc.M109.047829 
Priori, S. G., Wilde, A. A., Horie, M., Cho, Y., Behr, E. R., Berul, C., et al. (2013). HRS/EHRA/APHRS expert consensus statement on the diagnosis and management of patients with inherited primary arrhythmia syndromes: document endorsed by HRS, EHRA, and APHRS in May 2013 and by ACCF, AHA, PACES, and AEPC in June 2013. Heart Rhythm. 10, 19321963. doi: 10.1016/j.hrthm.2013.05.014

Pusch, M., Magrassi, R., Wollnik, B., and Conti, F. (1998). Activation and inactivation of homomeric KvLQT1 potassium channels. Biophys. J. 75, 785792. doi: 10.1016/S0006-3495(98)77568-X

Pusch, M., Bertorello, L., and Conti, F. (2000). Gating and flickery block differentially affected by rubidium in homomeric KCNQ1 and heteromeric KCNQ1/KCNE1 potassium channels. Biophys. J. 78, 211-226. doi: 10.1016/ S0006-3495(00)76586-6

Qu, Y., Isom, L. L., Westenbroek, R. E., Rogers, J. C., Tanada, T. N., Mccormick, K. A., et al. (1995). Modulation of cardiac Na+ channel expression in Xenopus oocytes by beta 1 subunits. J. Biol. Chem. 270, 25696-25701. doi: 10.1074/ jbc.270.43.25696

Ragsdale, D. S., Mcphee, J. C., Scheuer, T., and Catterall, W. A. (1994). Molecular determinants of state-dependent block of $\mathrm{Na}+$ channels by local anesthetics. Science 265, 1724-1728. doi: 10.1126/science. 8085162

Ragsdale, D. S., Mcphee, J. C., Scheuer, T., and Catterall, W. A. (1996). Common molecular determinants of local anesthetic, antiarrhythmic, and anticonvulsant block of voltage-gated Na+ channels. Proc. Natl. Acad. Sci. U. S. A 93, 92709275. doi: 10.1073/pnas.93.17.9270

Ramos, E., and O'leary M, E. (2004). State-dependent trapping of flecainide in the cardiac sodium channel. J. Physiol. 560, 37-49. doi: 10.1113/ jphysiol.2004.065003

Rampe, D., Murawsky, M. K., Grau, J., and Lewis, E. W. (1998). The antipsychotic agent sertindole is a high affinity antagonist of the human cardiac potassium channel HERG. J. Pharmacol. Exp. Ther. 286, 788-793.

Riuro, H., Campuzano, O., Arbelo, E., Iglesias, A., Batlle, M., Perez-Villa, F., et al. (2014). A missense mutation in the sodium channel betalb subunit reveals SCN1B as a susceptibility gene underlying long QT syndrome. Heart Rhythm. 11, 1202-1209. doi: 10.1016/j.hrthm.2014.03.044

Rocheleau, J. M., and Kobertz, W. R. (2008). KCNE peptides differently affect voltage sensor equilibrium and equilibration rates in KCNQ1 K+ channels. J. Gen. Physiol. 131, 59-68. doi: 10.1085/jgp.200709816

Roden, D. M., Woosley, R. L., and Primm, R. K. (1986). Incidence and clinical features of the quinidine-associated long QT syndrome: implications for patient care. Am. Heart J. 111, 1088-1093. doi: 10.1016/0002-8703(86) 90010-4

Roepke, T. K., Anantharam, A., Kirchhoff, P., Busque, S. M., Young, J. B., Geibel, J. P., et al. (2006). The KCNE2 potassium channel ancillary subunit is essential for gastric acid secretion. J. Biol. Chem. 281, 23740-23747. doi: 10.1074/ jbc.M604155200

Ruscic, K. J., Miceli, F., Villalba-Galea, C. A., Dai, H., Mishina, Y., Bezanilla, F., et al. (2013). IKs channels open slowly because KCNE1 accessory subunits slow the movement of S4 voltage sensors in KCNQ1 pore-forming subunits. Proc. Natl. Acad. Sci. U. S. A 110, E559-E566. doi: 10.1073/pnas.1222616110

Sachyani, D., Dvir, M., Strulovich, R., Tria, G., Tobelaim, W., Peretz, A., et al. (2014). Structural basis of a Kv7.1 potassium channel gating module: studies of the intracellular c-terminal domain in complex with calmodulin. Structure 22, 1582-1594. doi: 10.1016/j.str.2014.07.016

Salata, J. J., Jurkiewicz, N. K., Wang, J., Evans, B. E., Orme, H. T., and Sanguinetti, M. C. (1998). A novel benzodiazepine that activates cardiac slow delayed rectifier K+ currents. Mol. Pharmacol. 54, 220-230. doi: 10.1124/mol.54.1.220

Sanguinetti, M. C., Curran, M. E., Zou, A., Shen, J., Spector, P. S., Atkinson, D. L., et al. (1996). Coassembly of K(v)LQT1 and minK (IsK) proteins to form cardiac I-Ks potassium channel. Nature 384, 80-83. doi: 10.1038/384080a0

Saxena, P., Zangerl-Plessl, E. M., Linder, T., Windisch, A., Hohaus, A., Timin, E., et al. (2016). New potential binding determinant for hERG channel inhibitors. Sci. Rep. 6, 24182. doi: 10.1038/srep24182

Schoppa, N. E., Mccormack, K., Tanouye, M. A., and Sigworth, F. J. (1992). The size of gating charge in wild-type and mutant Shaker potassium channels. Science 255, 1712-1715. doi: 10.1126/science. 1553560

Schroeder, B. C., Waldegger, S., Fehr, S., Bleich, M., Warth, R., Greger, R., et al. (2000). A constitutively open potassium channel formed by KCNQ1 and KCNE3. Nature 403, 196-199. doi: 10.1038/35003200
Schwake, M., Jentsch, T. J., and Friedrich, T. (2003). A carboxy-terminal domain determines the subunit specificity of KCNQ K+ channel assembly. EMBO Rep. 4, 76-81. doi: 10.1038/sj.embor.embor715

Schwake, M., Athanasiadu, D., Beimgraben, C., Blanz, J., Beck, C., Jentsch, T. J., et al. (2006). Structural determinants of M-type KCNQ (Kv7) K+ channel assembly. J. Neurosci. 26, 3757-3766. doi: 10.1523/JNEUROSCI.5017-05.2006

Schwartz, P. J., Priori, S. G., Spazzolini, C., Moss, A. J., Vincent, G. M., Napolitano, C., et al. (2001). Genotype-phenotype correlation in the long-QT syndrome: gene-specific triggers for life-threatening arrhythmias. Circulation 103, 89-95. doi: 10.1161/01.CIR.103.1.89

Schwartz, P. J., Stramba-Badiale, M., Crotti, L., Pedrazzini, M., Besana, A., Bosi, G., et al. (2009). Prevalence of the congenital long-QT syndrome. Circulation 120, 1761-1767. doi: 10.1161/CIRCULATIONAHA.109.863209

Schwartz, P. J., Crotti, L., and Insolia, R. (2012). Long-QT syndrome: from genetics to management. Circ. Arrhythm Electrophysiol. 5, 868-877. doi: 10.1161/ CIRCEP.111.962019

Seebohm, G., Chen, J., Strutz, N., Culberson, C., Lerche, C., and Sanguinetti, M. C. (2003a). Molecular determinants of KCNQ1 channel block by a benzodiazepine. Mol. Pharmacol. 64, 70-77. doi: 10.1124/mol.64.1.70

Seebohm, G., Pusch, M., Chen, J., and Sanguinetti, M. C. (2003b). Pharmacological activation of normal and arrhythmia-associated mutant KCNQ1 potassium channels. Circ. Res. 93, 941-947. doi: 10.1161/01.RES.0000102866.67863.2B

Seebohm, G., Sanguinetti, M. C., and Pusch, M. (2003c). Tight coupling of rubidium conductance and inactivation in human KCNQ1 potassium channels. J. Physiol. London 552, 369-378. doi: 10.1113/jphysiol.2003.046490

Seebohm, G., Westenskow, P., Lang, F., and Sanguinetti, M. C. (2005). Mutation of colocalized residues of the pore helix and transmembrane segments S5 and S6 disrupt deactivation and modify inactivation of KCNQ1 K+ channels. J. Physiol. 563, 359-368. doi: 10.1113/jphysiol.2004.080887

Seebohm, G., Strutz-Seebohm, N., Ureche, O. N., Baltaev, R., Lampert, A., Kornichuk, G., et al. (2006). Differential roles of S6 domain hinges in the gating of KCNQ potassium channels. Biophys. J. 90, 2235-2244. doi: 10.1529/biophysj.105.067165

Selyanko, A. A., Hadley, J. K., Wood, I. C., Abogadie, F. C., Jentsch, T. J., and Brown, D. A. (2000). Inhibition of KCNQ1-4 potassium channels expressed in mammalian cells via M1 muscarinic acetylcholine receptors. J. Physiol. 522 (Pt 3), 349-355. doi: 10.1111/j.1469-7793.2000.t01-2-00349.x

Seoh, S. A., Sigg, D., Papazian, D. M., and Bezanilla, F. (1996). Voltage-sensing residues in the S2 and S4 segments of the Shaker K+ channel. Neuron 16, 11591167. doi: 10.1016/S0896-6273(00)80142-7

Sesti, F., Abbott, G. W., Wei, J., Murray, K. T., Saksena, S., Schwartz, P. J., et al. (2000). A common polymorphism associated with antibiotic-induced cardiac arrhythmia. Proc. Natl. Acad. Sci. U. States America 97, 10613-10618. doi: 10.1073/pnas. 180223197

Shen, H., Zhou, Q., Pan, X., Li, Z., Wu, J., and Yan, N. (2017). Structure of a eukaryotic voltage-gated sodium channel at near-atomic resolution. Science 355, pii: eaal4326. doi: $10.1126 /$ science.aal 4326

Shen, H., Liu, D., Wu, K., Lei, J., and Yan, N. (2019). Structures of human Nav1.7 channel in complex with auxiliary subunits and animal toxins. Science 363, 1303-1308. doi: 10.1126/science.aaw2493

Shimizu, W., and Antzelevitch, C. (1998). Cellular basis for the ECG features of the LQT1 form of the long-QT syndrome: effects of beta-adrenergic agonists and antagonists and sodium channel blockers on transmural dispersion of repolarization and torsade de pointes. Circulation 98, 2314-2322. doi: 10.1161/01.CIR.98.21.2314

Shimizu, W., Horie, M., Ohno, S., Takenaka, K., Yamaguchi, M., Shimizu, M., et al. (2004). Mutation site-specific differences in arrhythmic risk and sensitivity to sympathetic stimulation in the LQT1 form of congenital long QT syndrome: multicenter study in Japan. J. Am. Coll. Cardiol. 44, 117-125. doi: 10.1016/ j.jacc.2004.03.043

Shy, D., Gillet, L., and Abriel, H. (2013). Cardiac sodium channel NaV1.5 distribution in myocytes via interacting proteins: the multiple pool model. Biochim. Biophys. Acta 1833, 886-894. doi: 10.1016/j.bbamcr.2012.10.026

Skinner, J. R., Winbo, A., Abrams, D., Vohra, J., and Wilde, A. A. (2019). Channelopathies That Lead to Sudden Cardiac Death: Clinical and Genetic Aspects. Heart Lung Circ. 28, 22-30. doi: 10.1016/j.hlc.2018.09.007

Smith, P. L., Baukrowitz, T., and Yellen, G. (1996). The inward rectification mechanism of the HERG cardiac potassium channel. Nature 379, 833-836. doi: $10.1038 / 379833 \mathrm{a} 0$ 
Smith, J. L., Anderson, C. L., Burgess, D. E., Elayi, C. S., January, C. T., and Delisle, B. P. (2016). Molecular pathogenesis of long QT syndrome type 2. J. Arrhythm 32, 373-380. doi: 10.1016/j.joa.2015.11.009

Sokolov, S., Scheuer, T., and Catterall, W. A. (2007). Gating pore current in an inherited ion channelopathy. Nature 446, 76-78. doi: 10.1038/nature05598

Stansfeld, P. J., Grottesi, A., Sands, Z. A., Sansom, M. S., Gedeck, P., Gosling, M., et al. (2008). Insight into the mechanism of inactivation and $\mathrm{pH}$ sensitivity in potassium channels from molecular dynamics simulations. Biochemistry 47, 7414-7422. doi: 10.1021/bi800475j

Stenson, P. D., Ball, E. V., Mort, M., Phillips, A. D., Shaw, K., and Cooper, D. N. (2012). The Human Gene Mutation Database (HGMD) and its exploitation in the fields of personalized genomics and molecular evolution. Curr. Protoc. Bioinf. Chapter 1, Unit1 13. 1.13.1-1.13.20 doi: 10.1002/0471250953.bi0113s39

Strutz-Seebohm, N., Pusch, M., Wolf, S., Stoll, R., Tapken, D., Gerwert, K., et al. (2011). Structural basis of slow activation gating in the cardiac I Ks channel complex. Cell Physiol. Biochem. 27, 443-452. doi: 10.1159/000329965

Sun, J., and Mackinnon, R. (2017). Cryo-EM Structure of a KCNQ1/CaM Complex Reveals Insights into Congenital Long QT Syndrome. Cell 169, 1042-1050.e1049. doi: 10.1016/j.cell.2017.05.019

Sun, J., and Mackinnon, R. (2020). Structural Basis of Human KCNQ1 Modulation and Gating. Cell 180, 340-347 e349. doi: 10.1016/j.cell.2019.12.003

Sunami, A., Dudley, S. C.Jr., and Fozzard, H. A. (1997). Sodium channel selectivity filter regulates antiarrhythmic drug binding. Proc. Natl. Acad. Sci. U. S. A 94, 14126-14131. doi: 10.1073/pnas.94.25.14126

Tao, X., Lee, A., Limapichat, W., Dougherty, D. A., and Mackinnon, R. (2010). A gating charge transfer center in voltage sensors. Science 328, 67-73. doi: 10.1126/science. 1185954

Tapper, A. R., and George, A. L. (2001). Location and orientation of minK within the I-Ks potassium channel complex. J. Biol. Chem. 276, 38249-38254. doi: 10.1074/jbc.M103956200

Taylor, K. C., and Sanders, C. R. (2017). Regulation of KCNQ/Kv7 family voltagegated K(+) channels by lipids. Biochim. Biophys. Acta Biomembr 1859, 586597. doi: 10.1016/j.bbamem.2016.10.023

Taylor, K. C., Kang, P. W., Hou, P., Yang, N.-D., Kuenze, G., Smith, J. A., et al. (2020). Structure and Physiological Function of the Human KCNQ1 Channel Voltage Sensor Intermediate State. eLife 9, e53901. doi: 10.7554/eLife.53901

Terrenoire, C., Wang, K., Tung, K. W., Chung, W. K., Pass, R. H., Lu, J. T., et al. (2013). Induced pluripotent stem cells used to reveal drug actions in a long QT syndrome family with complex genetics. J. Gen. Physiol. 141, 61-72. doi: 10.1085/jgp.201210899

Tester, D. J., and Ackerman, M. J. (2014). Genetics of long QT syndrome. Methodist Debakey Cardiovasc. J. 10, 29-33. doi: 10.14797/mdcj-10-1-29

Thomas, D., Khalil, M., Alter, M., Schweizer, P. A., Karle, C. A., Wimmer, A. B., et al. (2010). Biophysical characterization of KCNQ1 P320 mutations linked to long QT syndrome 1. J. Mol. Cell Cardiol. 48, 230-237. doi: 10.1016/ j.yjmcc.2009.06.009

Thomas, A. M., Harmer, S. C., Khambra, T., and Tinker, A. (2011). Characterization of a binding site for anionic phospholipids on KCNQ1. J. Biol. Chem. 286, 2088-2100. doi: 10.1074/jbc.M110.153551

Tinel, N., Diochot, S., Borsotto, M., Lazdunski, M., and Barhanin, J. (2000). KCNE2 confers background current characteristics to the cardiac KCNQ1 potassium channel. EMBO J. 19, 6326-6330. doi: 10.1093/emboj/19.23.6326

Tobelaim, W. S., Dvir, M., Lebel, G., Cui, M., Buki, T., Peretz, A., et al. (2017a). Ca (2+)-Calmodulin and PIP2 interactions at the proximal C-terminus of Kv7 channels. Channels (Austin) 11, 686-695. doi: 10.1080/19336950.2017.1388478

Tobelaim, W. S., Dvir, M., Lebel, G., Cui, M., Buki, T., Peretz, A., et al. (2017b). Competition of calcified calmodulin N lobe and PIP2 to an LQT mutation site in Kv7.1 channel. Proc. Natl. Acad. Sci. U. S. A 114, E869-E878. doi: 10.1073/ pnas. 1612622114

Toh, M. F., Brooks, J. M., Strassmaier, T., Haedo, R. J., Puryear, C. B., Roth, B. L., et al. (2020). Application of High-Throughput Automated Patch-Clamp Electrophysiology to Study Voltage-Gated Ion Channel Function in Primary Cortical Cultures. SLAS Discovery, 2472555220902388. doi: 10.1177/ 2472555220902388

Toombes, G. E., and Swartz, K. J. (2016). STRUCTURAL BIOLOGY. Twists and turns in gating ion channels with voltage. Science 353, 646-647. doi: 10.1126/ science.aah4194
Tsang, S. Y., Tsushima, R. G., Tomaselli, G. F., Li, R. A., and Backx, P. H. (2005). A multifunctional aromatic residue in the external pore vestibule of $\mathrm{Na}+$ channels contributes to the local anesthetic receptor. Mol. Pharmacol. 67, 424-434. doi: 10.1124/mol.67.2.424

Ulmschneider, M. B., Bagneris, C., Mccusker, E. C., Decaen, P. G., Delling, M., Clapham, D. E., et al. (2013). Molecular dynamics of ion transport through the open conformation of a bacterial voltage-gated sodium channel. Proc. Natl. Acad. Sci. U. S. A 110, 6364-6369. doi: 10.1073/pnas.1214667110

Urrutia, J., Aguado, A., Muguruza-Montero, A., Nunez, E., Malo, C., Casis, O., et al. (2019). The Crossroad of Ion Channels and Calmodulin in Disease. Int. J. Mol. Sci. 20, pii: E400. doi: 10.3390/ijms20020400

Vaidyanathan, R., Reilly, L., and Eckhardt, L. L. (2018). Caveolin-3 Microdomain: Arrhythmia Implications for Potassium Inward Rectifier and Cardiac Sodium Channel. Front. Physiol. 9, 1548. doi: 10.3389/fphys.2018.01548

Valdivia, C. R., Medeiros-Domingo, A., Ye, B., Shen, W. K., Algiers, T. J., Ackerman, M. J., et al. (2010). Loss-of-function mutation of the SCN3Bencoded sodium channel \{beta\} 3 subunit associated with a case of idiopathic ventricular fibrillation. Cardiovasc. Res. 86, 392-400. doi: 10.1093/cvr/ cvp417

Van Horn, W. D., Vanoye, C. G., and Sanders, C. R. (2011). Working model for the structural basis for KCNE1 modulation of the KCNQ1 potassium channel. Curr. Opin. Struct. Biol. 21, 283-291. doi: 10.1016/j.sbi.2011.01.001

Vandenberg, J. I., Varghese, A., Lu, Y., Bursill, J. A., Mahaut-Smith, M. P., and Huang, C. L. (2006). Temperature dependence of human ether-a-go-go-related gene K+ currents. Am. J. Physiol. Cell Physiol. 291, C165-C175. doi: 10.1152/ ajpcell.00596.2005

Vandenberg, J. I., Perry, M. D., Perrin, M. J., Mann, S. A., Ke, Y., and Hill, A. P. (2012). hERG $\mathrm{K}(+)$ channels: structure, function, and clinical significance. Physiol. Rev. 92, 1393-1478. doi: 10.1152/physrev.00036.2011

Vandenberg, J. I., Perozo, E., and Allen, T. W. (2017). Towards a Structural View of Drug Binding to hERG K(+) Channels. Trends Pharmacol. Sci. 38, 899-907. doi: 10.1016/j.tips.2017.06.004

Vanoye, C. G., Desai, R. R., Fabre, K. L., Gallagher, S. L., Potet, F., Dekeyser, J. M., et al. (2018). High-Throughput Functional Evaluation of KCNQ1 Decrypts Variants of Unknown Significance. Circ. Genom. Precis Med. 11, e002345. doi: 10.1161/CIRCGEN.118.002345

Vardanyan, V., and Pongs, O. (2012). Coupling of voltage-sensors to the channel pore: a comparative view. Front. Pharmacol. 3, 145. doi: 10.3389/ fphar.2012.00145

Viswanathan, P. C., Shaw, R. M., and Rudy, Y. (1999). Effects of IKr and IKs heterogeneity on action potential duration and its rate dependence: a simulation study. Circulation 99, 2466-2474. doi: 10.1161/01.CIR.99.18.2466

Vitola, J., Vukanovic, J., and Roden, D. M. (1998). Cisapride-induced torsades de pointes. J. Cardiovasc. Electrophysiol. 9, 1109-1113. doi: 10.1111/j.15408167.1998.tb00888.x

Wacker, S., Noskov, S. Y., and Perissinotti, L. L. (2017). Computational Models for Understanding of Structure, Function and Pharmacology of the Cardiac Potassium Channel Kv11.1 (hERG). Curr. Top. Med. Chem. 17, 2681-2702. doi: 10.2174/1568026617666170414143430

Wallace, E., Howard, L., Liu, M., O'brien, T., Ward, D., Shen, S., et al. (2019). Long QT Syndrome: Genetics and Future Perspective. Pediatr. Cardiol. 40, 14191430. doi: $10.1007 / \mathrm{s} 00246-019-02151-\mathrm{x}$

Wang, W., and Mackinnon, R. (2017). Cryo-EM Structure of the Open Human Ether-a-go-go-Related $\mathrm{K}(+)$ Channel hERG. Cell 169, 422-430 e410. doi: 10.1016/j.cell.2017.03.048

Wang, Z., Tristani-Firouzi, M., Xu, Q., Lin, M., Keating, M. T., and Sanguinetti, M. C. (1999). Functional effects of mutations in KvLQT1 that cause long QT syndrome. J. Cardiovasc. Electrophysiol. 10, 817-826. doi: 10.1111/j.15408167.1999.tb00262.x

Wang, L., Dennis, A. T., Trieu, P., Charron, F., Ethier, N., Hebert, T. E., et al. (2009). Intracellular potassium stabilizes human ether-a-go-go-related gene channels for export from endoplasmic reticulum. Mol. Pharmacol. 75, 927937. doi: $10.1124 / \mathrm{mol} .108 .053793$

Wang, P., Yang, Q., Wu, X., Yang, Y., Shi, L., Wang, C., et al. (2010). Functional dominant-negative mutation of sodium channel subunit gene SCN3B associated with atrial fibrillation in a Chinese GeneID population. Biochem. Biophys. Res. Commun. 398, 98-104. doi: 10.1016/j.bbrc.2010.06.042 
Wang, Y. H., Jiang, M., Xu, X. L., Hsu, K.-L., Zhang, M., and Tseng, G.-N. (2011). Gating-related molecular motions in the extracellular domain of the IKs channel: implications for IKs channelopathy. J. Membr. Biol. 239, 137-156. doi: 10.1007/s00232-010-9333-7

Wang, C., Chung, B. C., Yan, H., Lee, S. Y., and Pitt, G. S. (2012). Crystal structure of the ternary complex of a NaV C-terminal domain, a fibroblast growth factor homologous factor, and calmodulin. Structure 20, 1167-1176. doi: 10.1016/ j.str.2012.05.001

Watanabe, H., Koopmann, T. T., Le Scouarnec, S., Yang, T., Ingram, C. R., Schott, J. J., et al. (2008). Sodium channel betal subunit mutations associated with Brugada syndrome and cardiac conduction disease in humans. J. Clin. Invest. 118, 2260-2268. doi: 10.1172/JCI33891

Watanabe, H., Darbar, D., Kaiser, D. W., Jiramongkolchai, K., Chopra, S., Donahue, B. S., et al. (2009). Mutations in sodium channel beta1- and beta2subunits associated with atrial fibrillation. Circ. Arrhythm Electrophysiol. 2, 268-275. doi: 10.1161/CIRCEP.108.779181

Weerapura, M., Nattel, S., Chartier, D., Caballero, R., and Hebert, T. E. (2002). A comparison of currents carried by HERG, with and without coexpression of MiRP1, and the native rapid delayed rectifier current. Is MiRP1 the missing link? J. Physiol. 540, 15-27. doi: 10.1113/jphysiol.2001.013296

Whicher, J. R., and Mackinnon, R. (2016). Structure of the voltage-gated K(+) channel Eag1 reveals an alternative voltage sensing mechanism. Science 353, 664-669. doi: 10.1126/science.aaf8070

Wiener, R., Haitin, Y., Shamgar, L., Fernandez-Alonso, M. C., Martos, A., Chomsky-Hecht, O., et al. (2008). The KCNQ1 (Kv7.1) COOH terminus, a multitiered scaffold for subunit assembly and protein interaction. J. Biol. Chem. 283, 5815-5830. doi: 10.1074/jbc.M707541200

Wilde, A., and Amin, A. S. (2018). Clinical Spectrum of SCN5A Mutations: Long QT Syndrome, Brugada Syndrome, and Cardiomyopathy. JACC Clin. Electrophysiol. 4, 569-579. doi: 10.1016/j.jacep.2018.03.006

Wisedchaisri, G., Tonggu, L., Mccord, E., Gamal El-Din, T. M., Wang, L., Zheng, N., et al. (2019). Resting-State Structure and Gating Mechanism of a VoltageGated Sodium Channel. Cell 178, 993-1003 e1012. doi: 10.1016/ j.cell.2019.06.031

Wu, L., Shryock, J. C., Song, Y., Li, Y., Antzelevitch, C., and Belardinelli, L. (2004). Antiarrhythmic effects of ranolazine in a guinea pig in vitro model of long-QT syndrome. J. Pharmacol. Exp. Ther. 310, 599-605. doi: 10.1124/jpet.104.066100

Wu, L., Yong, S. L., Fan, C., Ni, Y., Yoo, S., Zhang, T., et al. (2008). Identification of a new co-factor, MOG1, required for the full function of cardiac sodium channel Nav 1.5. J. Biol. Chem. 283, 6968-6978. doi: 10.1074/jbc.M709721200

Wu, D., Delaloye, K., Zaydman, M. A., Nekouzadeh, A., Rudy, Y., and Cui, J. (2010a). State-dependent electrostatic interactions of S4 arginines with E1 in S2 during Kv7.1 activation. J. Gen. Physiol. 135, 595-606. doi: 10.1085/ jgp.201010408

Wu, D., Pan, H., Delaloye, K., and Cui, J. (2010b). KCNE1 remodels the voltage sensor of Kv7.1 to modulate channel function. Biophys. J. 99, 3599-3608. doi: 10.1016/j.bpj.2010.10.018

Wu, J., Ding, W. G., and Horie, M. (2016). Molecular pathogenesis of long QT syndrome type 1. J. Arrhythm 32, 381-388. doi: 10.1016/j.joa.2015.12.006

Xiao, Y. F., Wright, S. N., Wang, G. K., Morgan, J. P., and Leaf, A. (1998). Fatty acids suppress voltage-gated $\mathrm{Na}+$ currents in HEK293t cells transfected with the alpha-subunit of the human cardiac Na+ channel. Proc. Natl. Acad. Sci. U. S. A 95, 2680-2685. doi: 10.1073/pnas.95.5.2680

Xiao, Y. F., Ke, Q., Wang, S. Y., Auktor, K., Yang, Y., Wang, G. K., et al. (2001). Single point mutations affect fatty acid block of human myocardial sodium channel alpha subunit Na+ channels. Proc. Natl. Acad. Sci. U. S. A 98, 36063611. doi: 10.1073/pnas.061003798

Xu, X., Jiang, M., Hsu, K.-L., Zhang, M., and Tseng, G.-N. (2008). KCNQ1 and KCNE1 in the IKs channel complex make state-dependent contacts in their extracellular domains. J. Gen. Physiol. 131, 589-603. doi: 10.1085/jgp.200809976

Xu, Y., Wang, Y., Meng, X.-Y., Zhang, M., Jiang, M., Cui, M., et al. (2013). Building KCNQ1/KCNE1 channel models and probing their interactions by moleculardynamics simulations. Biophys. J. 105, 2461-2473. doi: 10.1016/j.bpj.2013.09.058

Xu, Y., Wang, Y., Zhang, M., Jiang, M., Rosenhouse-Dantsker, A., Wassenaar, T., et al. (2015). Probing binding sites and mechanisms of action of an I(Ks) activator by computations and experiments. Biophys. J. 108, 62-75. doi: 10.1016/j.bpj.2014.10.059
Xu, H., Li, T., Rohou, A., Arthur, C. P., Tzakoniati, F., Wong, E., et al. (2019). Structural Basis of Nav1.7 Inhibition by a Gating-Modifier Spider Toxin. Cell 176702-715, e714. doi: 10.1016/j.cell.2018.12.018

Yan, H., Wang, C., Marx, S. O., and Pitt, G. S. (2017a). Calmodulin limits pathogenic Na+ channel persistent current. J. Gen. Physiol. 149, 277-293. doi: 10.1085/jgp.201611721

Yan, Z., Zhou, Q., Wang, L., Wu, J., Zhao, Y., Huang, G., et al. (2017b). Structure of the Nav1.4-betal Complex from Electric Eel. Cell 170, 470-482 e411. doi: 10.1016/j.cell.2017.06.039

Yang, W. P., Levesque, P. C., Little, W. A., Conder, M. L., Shalaby, F. Y., and Blanar, M. A. (1997). KvLQT1, a voltage-gated potassium channel responsible for human cardiac arrhythmias. Proc. Natl. Acad. Sci. U. S. A 94, 4017-4021. doi: 10.1073/pnas.94.8.4017

Yang, T., Smith, J. A., Leake, B. F., Sanders, C. R., Meiler, J., and Roden, D. M. (2013). An allosteric mechanism for drug block of the human cardiac potassium channel KCNQ1. Mol. Pharmacol. 83, 481-489. doi: 10.1124/mol.112.081513

Yang, J., Wang, Z., Sinden, D. S., Wang, X., Shan, B., Yu, X., et al. (2016). FGF13 modulates the gating properties of the cardiac sodium channel Nav1.5 in an isoform-specific manner. Channels (Austin) 10, 410-420. doi: 10.1080/ 19336950.2016.1190055

Yarov-Yarovoy, V., Brown, J., Sharp, E. M., Clare, J. J., Scheuer, T., and Catterall, W. A. (2001). Molecular determinants of voltage-dependent gating and binding of poreblocking drugs in transmembrane segment IIIS6 of the $\mathrm{Na}(+)$ channel alpha subunit. J. Biol. Chem. 276, 20-27. doi: 10.1074/jbc.M006992200

Yarov-Yarovoy, V., Mcphee, J. C., Idsvoog, D., Pate, C., Scheuer, T., and Catterall, W. A. (2002). Role of amino acid residues in transmembrane segments IS6 and IIS6 of the Na+ channel alpha subunit in voltage-dependent gating and drug block. J. Biol. Chem. 277, 35393-35401. doi: 10.1074/jbc.M206126200

Yus-Najera, E., Santana-Castro, I., and Villarroel, A. (2002). The identification and characterization of a noncontinuous calmodulin-binding site in noninactivating voltage-dependent KCNQ potassium channels. J. Biol. Chem. 277, 28545-28553. doi: 10.1074/jbc.M204130200

Zaydman, M. A., Silva, J. R., Delaloye, K., Li, Y., Liang, H., Larsson, H. P., et al. (2013). Kv7.1 ion channels require a lipid to couple voltage sensing to pore opening. Proc. Natl. Acad. Sci. U. S. A 110, 13180-13185. doi: 10.1073/pnas.1305167110

Zaydman, M. A., Kasimova, M. A., Mcfarland, K., Beller, Z., Hou, P., Kinser, H. E., et al. (2014). Domain-domain interactions determine the gating, permeation, pharmacology, and subunit modulation of the IKs ion channel. eLife 3, e03606. doi: 10.7554/eLife.03606

Zaza, A., Belardinelli, L., and Shryock, J. C. (2008). Pathophysiology and pharmacology of the cardiac "late sodium current". Pharmacol. Ther. 119, 326-339. doi: 10.1016/j.pharmthera.2008.06.001

Zhang, H., Craciun, L. C., Mirshahi, T., Rohacs, T., Lopes, C. M., Jin, T., et al. (2003). PIP(2) activates KCNQ channels, and its hydrolysis underlies receptormediated inhibition of M currents. Neuron 37, 963-975. doi: 10.1016/S08966273(03)00125-9

Zhang, M., Liu, J., and Tseng, G. N. (2004). Gating charges in the activation and inactivation processes of the HERG channel. J. Gen. Physiol. 124, 703-718. doi: 10.1085/jgp.200409119

Zhang, X. C., Yang, H., Liu, Z., and Sun, F. (2018). Thermodynamics of voltagegated ion channels. Biophys. Rep. 4, 300-319. doi: 10.1007/s41048-018-0074-y

Zhao, J. T., Hill, A. P., Varghese, A., Cooper, A. A., Swan, H., Laitinen-Forsblom, P. J., et al. (2009). Not all hERG pore domain mutations have a severe phenotype: G584S has an inactivation gating defect with mild phenotype compared to G572S, which has a dominant negative trafficking defect and a severe phenotype. J. Cardiovasc. Electrophysiol. 20, 923-930. doi: 10.1111/ j.1540-8167.2009.01468.x

Zhou, Z., Vorperian, V. R., Gong, Q., Zhang, S., and January, C. T. (1999). Block of HERG potassium channels by the antihistamine astemizole and its metabolites desmethylastemizole and norastemizole. J. Cardiovasc. Electrophysiol. 10, 836843. doi: 10.1111/j.1540-8167.1999.tb00264.x

Zhou, Y., Morais-Cabral, J. H., Kaufman, A., and Mackinnon, R. (2001). Chemistry of ion coordination and hydration revealed by a $\mathrm{K}+$ channel-Fab complex at 2.0 A resolution. Nature 414, 43-48. doi: 10.1038/35102009

Zhu, W., Voelker, T. L., Varga, Z., Schubert, A. R., Nerbonne, J. M., and Silva, J. R. (2017). Mechanisms of noncovalent beta subunit regulation of $\mathrm{NaV}$ channel gating. J. Gen. Physiol. 149 (8), 813-831. doi: 10.1085/jgp.201711802 
Zipes, D. P., Camm, A. J., Borggrefe, M., Buxton, A. E., Chaitman, B., Fromer, M., et al. (2006). ACC/AHA/ESC 2006 Guidelines for Management of Patients With Ventricular Arrhythmias and the Prevention of Sudden Cardiac Death: a report of the American College of Cardiology/American Heart Association Task Force and the European Society of Cardiology Committee for Practice Guidelines (writing committee to develop Guidelines for Management of Patients With Ventricular Arrhythmias and the Prevention of Sudden Cardiac Death): developed in collaboration with the European Heart Rhythm Association and the Heart Rhythm Society. Circulation 114, e385e484. doi: 10.1161/CIRCULATIONAHA.106.178233
Conflict of Interest: The authors declare that the research was conducted in the absence of any commercial or financial relationships that could be construed as a potential conflict of interest.

Copyright $\odot 2020$ Brewer, Kuenze, Vanoye, George, Meiler and Sanders. This is an openaccess article distributed under the tes of the Creative Commons Attribution License (CC BY). The use, distribution or reproduction in other forums is permitted, provided the original author(s) and the copyright owner(s) are credited and that the original publication in this journal is cited, in accordance with accepted academic practice. No use, distribution or reproduction is permitted which does not comply with these terms. 